\title{
Granular Electronic Systems
}

\author{
I. S. Beloborodov, A. V. Lopatin, and V. M. Vinokur \\ Materials Science Division, Argonne National Laboratory, Argonne, Illinois 60439, USA
}

K. B. Efetov

Theoretische Physik III, Ruhr-Universität Bochum, 44780 Bochum, Germany and L. D. Landau Institute for Theoretical Physics, 117940 Moscow, Russia.

\begin{abstract}
A granular metal is an array of metallic nano-particles imbedded into an insulating matrix. Tuning the intergranular coupling strength a granular system can be transformed into either a good metal or an insulator and, in case of superconducting particles, experience superconductor-insulator transition. The ease of adjusting electronic properties of granular metals makes them most suitable for fundamental studies of disordered solids and assures them a fundamental role for nanotechnological applications. This Review discusses recent important theoretical advances in the study of granular metals, emphasizing on the interplay of disorder, quantum effects, fluctuations and effects of confinement in formation of electronic transport and thermodynamic properties of granular materials.
\end{abstract}

\section{Contents}

I. Introduction

A. Why are granular electronic systems interesting?

B. Physical quantities characterizing granular materials

II. Normal granule arrays

A. Transport properties

1. Classical conductivitv

2. Metallic regime

3. Insulating regime

B. Model and main theoretical tools

1. Hamiltonian

2. Diagrammatic technique for granular metals

3. Coulomb interaction and gauge transformation

4. Ambegaokar-Eckern-Schön functional

C. Metallic properties of granular arravs at not verv low temperatures 16

1. Perturbation theorv

2. Renormalization Group 17

16

2. Renormalization Group 17

E. Universal description of granular materials

20

F. Insulating Properties of Granular Metals: Periodic model 22

1. Activation conductivity behavion

2. Mott gap at small tunneling conductances

3. Mott gap at large tunneling conductances

4. Metal - Insulator transition in periodic granular arravs 24

G. Hopping conductivity in Granular Materials

1. Density of states

2. Hopping via elastic co-tunneling

3. Hopping via inelastic co-tunneling

III. Arrays composed of superconducting grains

A. General properties of granular superconductors 1. Single grain

2. Macroscopic superconductivitv

3. Granular superconductor in a magnetic field 4. Transport properties of granular superconductors

B. Phase diagram of granular superconductors

1. Phase functional for the granular superconductors

2. Mean field approximation

C. Upper critical field of a granular superconductor

1. Critical field of a single grain

2. Critical magnetic field of a granular sample
D. Suppression of the superconducting critical temperature 38

E. Magnetoresistance of granular superconductors 40

IV. Discussion of the results

A. Quantitative comparison with experiments 43 1. Logarithmic temperature dependence of the conductivity 43

2. Hopping conductivity 44

3. Negative magnetoresistance $\quad 45$

\begin{tabular}{ll}
\hline B. Outlook & 45
\end{tabular}

Acknowledgements 46

A. Calculation of the tunneling probability $P_{e l}$ in the elastic regime

B. Calculation of the tunneling probability $P_{i n}$ in the inelastic regir References

49

\section{INTRODUCTION}

\section{A. Why are granular electronic systems interesting?}

Granular conductors form a new class of artificial materials with tunable electronic properties controlled at the nanoscale and composed of close-packed granules varying in size from a few to hundred nanometers (often referred to as nanocrystals). The granules are large enough to possess a distinct electronic structure, but sufficiently small to be mesoscopic in nature and exhibit effects of quantized electronic levels of confined electrons. Granular conductors combine the unique properties of individual- and the collective properties of coupled nanocrystals opening a new route for potential novel electronic, optical, and optoelectronic applications. Applications range from light emitting devices to photovoltaic cells and bio-sensors. The intense interest is motivated not only by the important technological promise but by the appeal of dealing with the experimentally accessible 
model system that is governed by tunable cooperative effects of disorder, electron correlations, and quantum phenomena (Gaponenko, 1998; Mowbrav and Skolnick, 2005; Murrav et al., 1993).

Among traditional methods of preparation of such materials, the most common are the thermal evaporation and sputtering techniques. During those processes metallic and insulating components are simultaneously evaporated or sputtered onto a substrate. Diffusion of metallic component leads to the formation of small metallic grains, usually 3-50 nm, see Fig. 1] Variations of grain sizes within a sample produced by these methods can be achieved to as low as about $\sim 10 \%$ (Gerber et al., 1997). Depending on the materials used for the preparation, one can obtain magnetic systems, superconductors, insulators, etc.

Recent years have seen a remarkable progress achieved in the design of granular conductors with the controllable structure parameters. Granules can be capped with organic (ligands) or inorganic molecules which connect and regulate the coupling between them. Altering the size and shape of granules one can regulate quantum confinement effects. In particular, tuning microscopic parameters one can vary the granular materials from being relatively good metals to pronounced insulators as a function of the strength of electron tunnelling couplings between conducting grains. This makes granular conductors a perfect exemplary system for studing metalinsulator transition and related phenomena.

One emerging technique to create granular systems is through self-assembling colloidal nanocrystals (Collier et al., 1998; Murrav et al., 2000). For instance (Lin et al., 2001) describe almost perfectly periodic two-dimensional arrays of monodisperse (with grain size variations within $\sim 5 \%$ ) gold nanoparticles covered by ligand molecules that play a role of an insulating layer. Such samples are produced via self-assembling of gold nanoparticles at the liquid-air interface during the evaporation of a colloidal droplet (Naravanan et al., 2004). By changing the experimental conditions, multilayers of nanocrystals can also be created, see Fig. 22 (Parthasarathv et al., 2004, 2001; Tran et al., 2005). Other important examples include Langmuir films of colloidal Ag (Collier et al., 1997; Du et al., 2002) and arrays of semiconductor quantum dots (Du et al., 2002; Murrav et al., 1993; Wehrenberg et al., 2002; Yu et al., 2004).

Of the range of techniques developed for the fabrication of semiconductor quantum dot arrays, the most successful appeared the self-assembled technique of epitaxial growth of two semiconductors having significantly different lattice constants. For the prototype system of InAs on GaAs, where the lattice mismatch is $7 \%$, InAs initially deposited on GaAs grows as a strained two-dimensional layer (referred to as the wetting layer) (Mowbrav and Skolnick, 2005). These materials along with $\operatorname{In}_{2} \mathrm{O}_{3}: S n$ (known as indium tin oxide, ITO) are the most widely studied systems. They
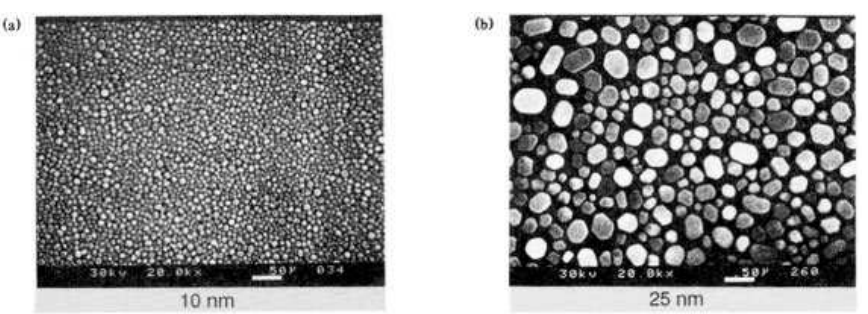

FIG. 1 Scanning electron microscope photographs of indium evaporated onto $\mathrm{SiO}_{2}$ at room temperatures (Yu et al.,1991).

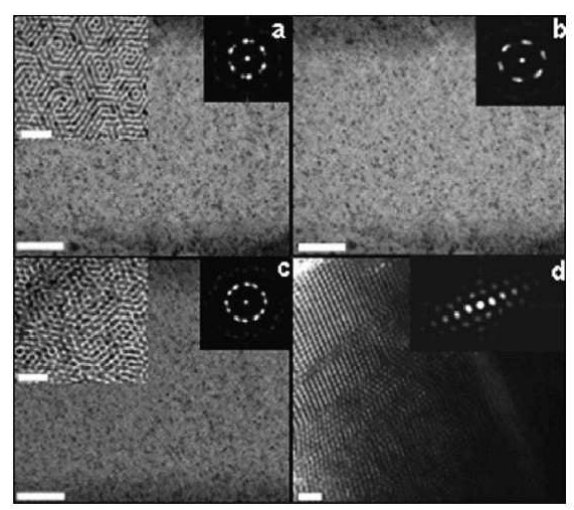

FIG. 2 Transmission electron micrographs showing periodic granular (a) bilayers, (b) trilayers, (c) tetralayers, and (d) thick films. The insets on the left sides of panels (a) and (c) are the zoomed-in images. The scale bars correspond to 200 $\mathrm{nm}$ (a)-(c) and $40 \mathrm{~nm}$ (d) (Tran et al., 2005)

exhibit a high visible transparency and a good electrical conductance. They are used as electrodes in light emitting diodes (Kim et al., 1998; Zhu et al., 2000) solar cells (Gordon, 2000), smart windows and flat panel displays.

All these experimental achievements and technological prospects call for a comprehensive theory able to provide quantitative description of transport and thermodynamic properties of granular conductors and can, therefore, serve as a ground for a clever design of devices for emerging new generation nano-electronics.

It has been realized for quite some time that granularity can bring new physics extending already wealthy list of remarkable effects exhibited by disordered systems even further. One of the early observation was the stretched exponential temperature behavior of the conductivity in the strongly disordered films and arrays of metallic granules in the insulating regime (see (Abeles et al., 1975) for a review):

$$
\sigma(T) \sim \exp \left(-\sqrt{T_{0} / T}\right),
$$

with $T_{0}$ being a material dependent constant. This behavior resembled the Mott-Efros-Shklovskii variable range hopping conductivity in semiconductors (Efros and Shklovskii, 1975; Shklovskii and Efros, 1988) and appeared to be generic for granular ar- 
rays both metallic and semiconducting - either irregular (Abeles et al., 1975) and strictly periodic (Romero and Drndid, 2005; Tran et al., 2005; Yu et al., 2004). Several explanations have been advanced for this striking behavior, but real understanding was achieved only recently (Beloborodov et al., 2005c; Feigel'man and Ioselevich, 2005; Tran et al., 2005; Zhang and Shklovskii, 2004).

A most recent incitement to intense and deep study of physics of granular materials was given by the influential works (Gerber et al., 1997; Simon et al., 1987) where a logarithmic dependence of the conductivity $\sigma(T)$

$$
\sigma(T)=a+b \ln T
$$

with $a$ and $b$ being material dependent constants, was observed in the metallic conductivity domain. This logarithmic behavior, has been observed in both two and three dimensional samples thus ruling out the tempting explanation in terms of the weak localization (Abrahams et al., 1979; Gorkov et al., 1979) or interaction corrections (Altshuler et al., 1980; Belitz and Kirkpatrick, 1994; Lee and Ramakrishnan, 1985) which result in logarithmic behavior in two dimensions only. The call for understanding these results brought to life new models (Beloborodov et al., 2003, 2004b; Efetov and Tschersich, 2002, 2003) which evolved into a new direction of research that will be one of the major topics of our review.

The feature that plays a fundamental role, especially at low temperatures, is the pronounced discreteness of the electronic levels due to electron confinement within a single grain. The mesoscopic scale of the grains brings about the levels statistics and all the wealth of the related effects (Halperin, 1986; Nagaev, 1992). The mean level spacing $\delta$ in a single grain is inversely proportional to the volume of the grain,

$$
\delta=(\nu V)^{-1}
$$

where $V$ is the volume of the grain and $\nu$ is the density of states at the Fermi energy. For metal particles of size of several nanometers, the parameter $\delta$ is typically of order of several Kelvin. For example, for an aluminum particle with radius $R=5 \mathrm{~nm}$ one has $\delta \sim 1 K$. However, we concentrate on the temperature range $T>\delta$ where the quantum size effects are not important. In fact both types of conductivity behavior, Eqs. (1.1) and (1.2), were observed at temperatures $T>\delta$.

Superconductivity brings yet another aspect to diversity of the interesting effects in granular materials (Gerber et al., 1997; Hadacek et al., 2004; Jaeger et al., 1989; Shapira and Deutscher, 1983). One of them is a counterintuitive suppression of the conductivity due to superconducting fluctuations.

In this review we summarize the recent theoretical progress in understanding of the phenomena observed in granular metals and superconductors. These are effects that do not demand ultra low temperatures (and

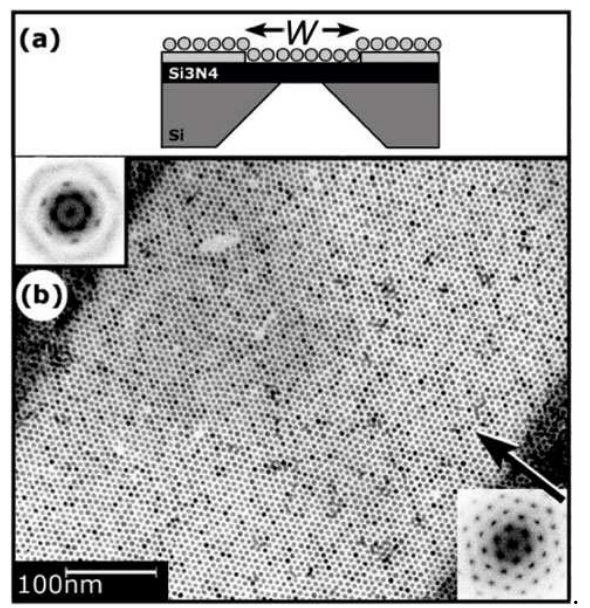

FIG. 3 a) Sketch of a nanocrystal monolayer and in-plane electrodes, b) Highly ordered superlattice between electrodes visible at the upper left and lower right. (Parthasarathv et al., 2001)

thus very sophisticated experimental setups) and are thus promising from the standpoint of possible applications. Physics of these phenomena is not particularly material specific and our consideration will be based on the correspondingly general models of the granular systems.

\section{B. Physical quantities characterizing granular materials}

This Review deals with the systems which we call a granular metals and which are well modelled by an array of the identical in size and shape but mesoscopically different metallic particles, the intergranular electron coupling being described via the tunnelling matrix. The grain arrangements may be either periodic or irregular.

The effect of irregularities in the grain positions and in the strengths of the tunneling coupling on the physical properties of granular systems is different for metallic and insulating samples. If the coupling between the grains is sufficiently strong and the system is well conducting, the irregularities are not very important. On the contrary, irregularities become crucial in the limit of low coupling where the system is an insulator. As a matter of practice, recent advances in fabrication techniques now permit very regular arrays with the fluctuations in the granule size within the $5 \%$ precision (Tran et al., 2005; Yu et al.,2004). As regular arrays promise many technological applications the further progress in making perfect systems is expected.

At the same time, disorder related to either internal defects inside and/or on the surface of the individual granules and to the charged impurities in the insulating substrate/matrix is unavoidable. Even in metallic grains with the mean free part exceeding their size, the electrons that move ballistically within the granules yet scatter at the grain boundaries irregularities; the eletfron motion becomes chaotic with the resulting effect equivalent to 
the action of the intragranular disorder (see, e.g. (Efetov, 1997)). This surface chaotization would have been absent in the atomically perfect spherical (or, say, cubical) granules. In such ideal granules the additional degeneracies of the energy levels would have led to singularities in physical quantities. However, the slightest deviations (even of the order of $1 \AA$ or of the order of the electron wavelength) of the shape of the grains from the ideal spheres or cubes would have lifted the accidental degeneracy of the levels (this would be equivalent to adding an internal disorder). Thus one can justly assume that the grains are always microscopically irregular. Moreover, the coupling between the grains is the source of the additional irregularities. We thus can adopt the model with the diffusive electron motion within the each grain without any loss of generality. In this model the use of expression of Eq. (1.3) for the mean level spacing $\delta$ is fully justified.

The key parameter that determines most of the physical properties of the granular array is the average tunnelling conductance $G$ between the neighboring grains. It is convenient to introduce the dimensionless conductance $g$ (corresponding to one spin component) measured in the units of the quantum conductance $e^{2} / \hbar: g=G /\left(2 e^{2} / \hbar\right)$. As we will see below, the samples with $g \gtrsim 1$ exhibit metallic transport properties, while those with $g \lesssim 1$ show an insulating behavior.

One of the most important energy parameters of the granular system is the single grain Coulomb charging energy $E_{c}$. This energy is equal to the change in the energy of the grain when adding or removing one electron, and it plays a crucial role in the transport properties in the insulating regime when electrons are localized in the grains, such that the charge of each grain is quantized. The physics of the insulating state is closely related to the well known phenomenon of the Coulomb blockade of a single grain connected to a metallic reservoir.

The behavior of a single grain in a contact with a reservoir has been discussed in many articles and reviews (see e.g. (Aleiner et al., 2002; Averin and Nazarov, 1992; Averin and Likharev, 1991)). The main features of the Coulomb blockade can be summarized as follows: (i) If the grain is weakly coupled to the metallic contact, $g \ll 1$, the charge on the grain is almost quantized; this is the regime of the so-called Coulomb blockade. (ii) In the opposite limit, $g \gg 1$, the effects of the charge quantization are negligible and the electrons freely exchange between the granule and reservoir.

Although the systems we consider are arrays of interconnected granules rather than a single grain coupled to a bulk metal, a somewhat similar behavior is expected: In the regime of the strong coupling between the grains, $g \gg 1$, electrons propagate easily through the granular sample and the Coulomb interaction is screened. In the opposite limit of the low coupling, $g \ll 1$, the charge on each grain gets quantized as in the standard Coulomb blockade behavior. In this case an electron has to overcome electrostatic barrier of the order $E_{c}$ in order to hop onto the neighboring granule. This impedes the trans- port at temperatures $T$ lower than $E_{c}$.

Throughout this review we will be always assuming that the mean level spacing $\delta$ from Eq. (1.3) is the smallest energy scale. In particular, in all cases we take the condition $E_{c} \gg \delta$ be satisfied. This is the most realistic condition when dealing with the metallic particles of a nanometer-size scale, given that the charging energy $E_{c}$ is inversely proportional to the radius $a$ of the grains, whereas the mean level spacing $\delta$ is inversely proportional to the volume $V$ and also taking into account the high density of energy states in metals. Note that this may not be the case in arrays of semiconductor dots, where $E_{c}$ and $\delta$ may appear of the same order, but this goes beyond the scope of our review.

Another important thing to remember is that the intergranular (tunneling) conductance $g$ is much smaller than the intragrain conductance $g_{0}$ by the very meaning of the notion of "granular system":

$$
g \ll g_{0}
$$

The intra-grain conductance $g_{0}$ is brought by scattering on impurities or on the boundaries of the grains and the inequality (1.4) means that the grains are not very dirty. The case $g \sim g_{0}$ can be viewed as a homogeneously disordered system and we do dwell on this limit here. The single grain conductance $g_{0}$ can be most easily defined as the physical conductance of a granule of a cubic geometry measured in the units of the quantum conductance $e^{2} / h$. In mesoscopic physics it is customary, however, to relate the single grain conductance $g_{0}$ to the single grain Thouless energy $E_{T h}$ as $g_{0}=E_{T h} / \delta$, where the energy $E_{T h}$ is defined as

$$
E_{T h}=D_{0} / a^{2}
$$

and $D_{0}=v_{F}^{2} \tau / d$ is the classical diffusion coefficient. Other parameters are $v_{F}$, the Fermi velocity, $\tau$, the elastic scattering time within the grains, and $d$, the dimensionality of the grain. The length $a$ in Eq. (1.5) is the linear size (radius) of the grain. If the grains are not very dirty, such that the electrons move inside the grains ballistically, the mean free path $l=v_{F} \tau$ should be replaced by the size of the grains $2 a$. The Thouless energy $E_{T h}$, Eq. (1.5), is proportional to the inverse time that it takes for an electron to traverse the grain. The energy $E_{T h}$, Eq. (1.5), exceeds the mean level spacing $\delta$, Eq. (1.3), and therefore the intragrain conductance $g_{0}$ is always large, $g_{0} \gg 1$, whereas the inter-granular conductance $g$ can be either larger or smaller than unity.

The above parameters make a full set of variables describing properties of the normal granular metals. If the constituent particles are made out of the superconductor material, a wealth of new interesting phenomena arises. The behavior of such a system can be quantified by adding one more energy parameter, the superconducting gap $\Delta$ of the material of a single granule. Now, if the inter-grain coupling is sufficiently strong, the system can turn a superconductor at sufficiently low temperatures. 
Properties of such a superconductor are not very different from those of bulk superconductors.

On the contrary, in the opposite limit of weak coupling between the granules, an array of superconducting grains can transform into an insulator at $T=0$. In this regime the Cooper pairs are locally formed in each grain but remain localized inside the grains due to the strong onsite Coulomb repulsion leading to the Coulomb blockade. Because of localization, the number of the Cooper pairs in each grain is fixed and, according to the uncertainty principle, this leads to strong phase fluctuations. Thus the system does not develop the global coherence, and the global macroscopic superconductivity is suppressed. One can describe this effect using the model of superconductor grains coupled via the Josephson junctions. Such a system is characterized by three energy parameters: the superconductor gap, $\Delta$, characterizing a single grain, the Josephson coupling $J$, and the grain charging Coulomb energy $E_{c}$. Strong Josephson coupling, $J \gg E_{c}$, suppresses the phase fluctuations leading to the globally coherent superconducting state at sufficiently low temperatures. If $J \ll E_{c}$, the Coulomb blockade prevails, the Cooper pairs get localized at $T \rightarrow 0$, and the system falls into an insulating state.

Note that even in the insulator state the superconducting gap $\Delta$ still exists in each grain and its value is close to the gap magnitude in the bulk provided $\Delta \gg \delta$. If the latter inequality is not fulfilled the superconducting gap in the grains is suppressed or can even be fully destroyed (Anderson, 1959, 1964). There are interesting effects in this regime but we do not consider them here because the relevant region of the parameters corresponds to either too small grains or too weak superconductors.

At low temperatures, the Josephson coupling $J$ is expressed via the tunnelling conductance $g$ as $J=\pi g \Delta / 2$ (Ambegaokar and Baratoff, 1963) and, at first glance, one could have concluded that the transition between the insulating and superconducting states should have happened at $g \sim E_{c} / \Delta$. However, this simple estimate holds only in the weak coupling regime, $g<1$, that assumes $E_{c}<\Delta$. At stronger coupling, the Coulomb energy $E_{c}$ is renormalized down to the value $E_{c} \rightarrow \tilde{E} \sim$ $\Delta / g$, Chakravartv et al., 1987: Larkin and Ovchinnikov, 1983) due to electron tunnelling between the neighboring grains. Therefore, in the limit of the strong coupling, $g \gg 1$, the effective Coulomb energy $\tilde{E}$ is always smaller than the Josephson coupling, $\tilde{E}<J$, implying the superconducting ground state. Shown in Fig. 4 is the schematic phase diagram summarizing the above consideration (Chakravartv et al., 1987). For many superconducting granular samples available experimentally the ratio $E_{c} / \Delta$ is large. In this case, as one can see from phase diagram in Fig. [4 the transition between the superconducting and insulating at $T \rightarrow 0$ occurs at $g \sim 1$ (Chakravartv et al., 1987; Orr et al., 1986).

To conclude our brief introduction to granular superconductors we note that for the most of experimentally available samples, the grains are much smaller than the

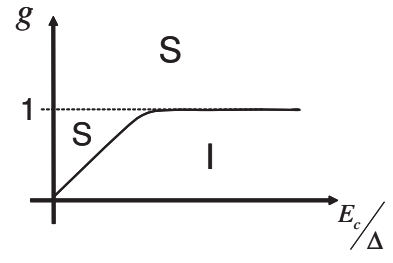

FIG. 4 Schematic phase diagram for granular superconductors at temperatures $T=0$. Symbols $S$ and $I$ stand for superconducting and insulating phases respectively.

bulk superconducting coherence length of the granule material

$$
a \ll \xi_{0} .
$$

This allows one to neglect variations of the superconducting order parameter $\Delta$ inside the grains and treat a single grain as a zero dimensional object. As the condition (1.6) is at this point the most common experimental situation, we will further concentrate mainly on this regime.

As we now see, depending on the parameters of the system, many different physical situations appear and the phase diagram of a granular material is quite reach. In the next two sections, Sec. II and Sec. [II] we will focus on the systems consisting of the normal and superconducting grains respectively.

\section{NORMAL GRANULE ARRAYS}

\section{A. Transport properties}

We start the discussion of the properties of the granular systems consisting of the normal grains by presenting the main results and their qualitative explanations. This may help the reader to understand the basic physics of the system and to learn important formulae suitable for a direct comparison with experiments without going into the technical detail of theoretical models, which will be presented at the subsequent parts of each section.

\section{Classical conductivity}

The key parameter that determines the transport properties of the granular materials is the tunneling conductance $g$. In the strong coupling regime, $g \gg 1$, a granular array has metallic properties, while in the opposite case, $g \ll 1$, the array is an insulator. The insulating state appears as a result of the strong Coulomb correlations that block electron transport at low temperatures. Generally speaking, apart from the Coulomb interaction effects one has also to consider the effects of quantum interference that may play an important role in the low conducting samples. In homogenously disordered systems the interference effects play an important role leading to the 
localization of electron states in the absence of interaction (Anderson, 1958).

In the metallic regime and at high enough temperatures both Coulomb correlation and interference effects are weak. In this case the global sample conductivity $\sigma_{0}$ is given by the classical Drude formula, in particular, for a periodic cubic granular array

$$
\sigma_{0}=2 e^{2} g a^{2-d},
$$

where $a$ is the size of the grains and $d$ is the dimensionality of the sample. Formula (2.1) has a straightforward meaning: in order to obtain the physical conductance of the contact, one should multiply its dimensionless conductance, $g$, by $2 e^{2}$ (the factor 2 is due to spin and $\hbar=1$ ) and, then, multiplying the result by $a^{2-d}$ one arrives at the conductivity per unit volume.

Although Eq. (2.1) is written for the periodic array, the electron system is not translationally invariant, otherwise the sample conductivity would be infinite. Equation (2.1) is valid for grains with an internal disorder, such that the electron motion inside the grains is chaotic. When hopping from grain to grain, the electron momentum is not conserved and this leads to the finite conductivity $\sigma_{0}$, Eq. 2.1). The conductance of the contact $g$ depends on the microscopic properties of the contact, and we will consider it in most cases as a phenomenological dimensionless parameter controlling behavior of the system. Note that the model of a periodic array assumes no variation in tunneling conductances.

Upon decreasing temperature the Coulomb interactions become relevant and formula Eq. (2.1) does not hold any more. Below we will discuss Coulomb effects in more detail and find that their manifestation in granular systems may differ noticeably from that in "homogeneously disordered" metals.

\section{Metallic regime}

In the metallic regime electrons tunnel easily from granule to granule. The time $\tau_{0}$ that the electron spends inside a grain plays an important role in the metallic regime: the corresponding characteristic energy $\Gamma=\tau_{0}^{-1}$ is related to the tunneling conductance and the mean energy level spacing as

$$
\Gamma=g \delta .
$$

The energy $\Gamma$ can also be interpreted as the width of the smearing of the energy levels in the grains. In the limit of large conductances, $g \gg 1$, this width exceeds the energy spacing $\delta$ and the discreteness of the levels within a single grain ceases to be relevant.

Since the electron motion on the scales well exceeding the granule size is always diffusive (even in the case of the ballistic electron motion inside each grain), the electron motion on the time scales larger than $\Gamma^{-1}$ can be described by the effective diffusion coefficient $D_{\text {eff }}$ related to $\Gamma$ as

$$
D_{\text {eff }}=\Gamma a^{2} .
$$

Then the Einstein relation gives the conductivity of the granular sample as

$$
\sigma_{0}=2 e^{2} \nu D_{e f f} .
$$

For periodic arrays Eq. (2.4) is equivalent to Eq. (2.1), which can be seen from Eqs. (1.3 2.2 2.3). At the same time Eq. (2.4) is more general than Eq. (2.1) since with the properly defined diffusion constant $D_{\text {eff }}$ it applies to arrays with the arbitrary grain arrangement as well.

The energy scale $\Gamma$ plays a very important role; many physical quantities have qualitatively different behavior depending on whether they are dominated by the energies higher or lower than $\Gamma$.

From the experience with the homogeneously disordered metals one can envision two major causes that may alter the classical conductivity $\sigma_{0}$ in Eq. (2.1): (i) electron-electron interactions (Altshuler and Aronov, 1985; Lee and Ramakrishnan, 1985) and (ii) quantum interference effects (Abrahams et al., 1979; Gorkov et al., 1979). Accordingly, constructing the theory of granular conductors with the reference of highly advanced theory of disordered metals, one can expect two corresponding distinct corrections to $\sigma_{0}$. Since in the metallic domain electrons effectively screen out the on-site Coulomb interactions the bare magnitudes of which are high because of small sizes of the grains, the notion of the interaction corrections to conductivity is well justified.

To gain the qualitative understanding of the interaction effects, we introduce the characteristic interaction temporal- and the corresponding spatial scales $\tau_{T} \sim \hbar / T$ and $L_{T} \sim \sqrt{D_{\text {eff }} / T}$ associated with this time. One expects that the behavior of the interaction correction is different on the distances exceeding the granule size, $L_{T}>a$, and within the granule, $L_{T}<a$. Using Eq. (2.3) for the effective diffusion coefficient $D_{\text {eff }}$, one immediately sees that these conditions imply the existence of two distinct temperature regions $T>\Gamma$ and $T<\Gamma$ with respect to interaction contributions. Accordingly, the correction to the conductivity due to Coulomb interaction can be written as a sum of contributions coming from the large, $\varepsilon>\Gamma$, and the low, $\varepsilon<\Gamma$, energies. This "contribution separation" naturally follows, as we will see below, from the diagrammatic approach where the two contributions in question are represented by the two distinct sets of the diagrams. Deferring the details for later, the result is as follows: denoting the corrections coming from the high and low with respect to $\Gamma$ energies as $\delta \sigma_{1}$ and $\delta \sigma_{2}$, respectively, we write:

$$
\sigma=\sigma_{0}+\delta \sigma_{1}+\delta \sigma_{2}
$$

with

$$
\frac{\delta \sigma_{1}}{\sigma_{0}}=-\frac{1}{2 \pi d g} \ln \left[\frac{g E_{c}}{\max (T, \Gamma)}\right]
$$




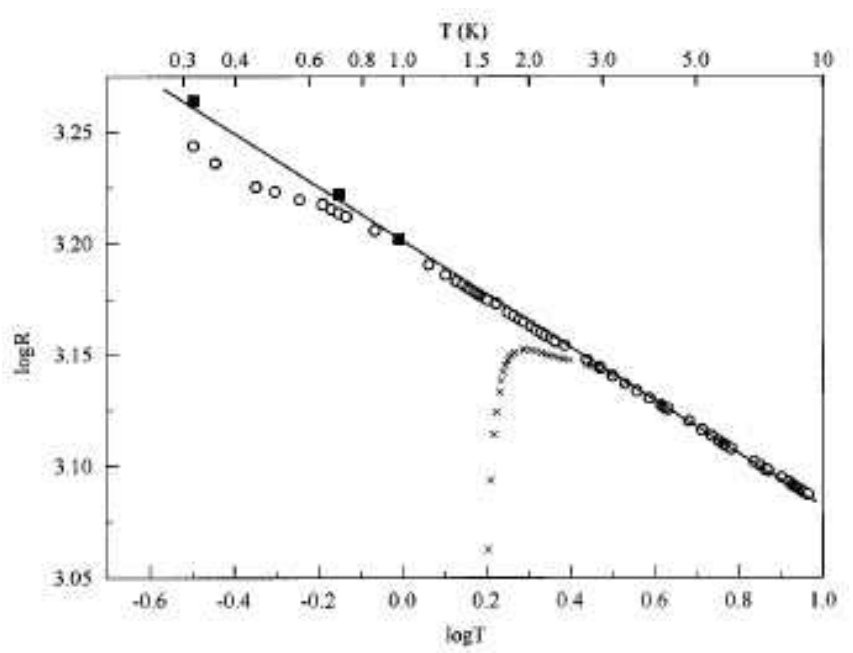

FIG. 5 Resistance of $3 d$ granular $A l-G e$ sample as a function of temperature on a log-log scale, as measured at (zero) $(x)$ and $100 \mathrm{kOe}$ field (open circles). Sample room temperature resistance is $500 \Omega$ (Gerber et al., 1997).

(Efetov and Tschersich, 2002, 2003) and

$$
\frac{\delta \sigma_{2}}{\sigma_{0}}= \begin{cases}\frac{\alpha}{12 \pi^{2} g} \sqrt{\frac{T}{\Gamma}} & d=3, \\ -\frac{1}{4 \pi^{2} g} \ln \frac{\Gamma}{T} & d=2, \\ -\frac{\beta}{4 \pi g} \sqrt{\frac{\Gamma}{T}} & d=1,\end{cases}
$$

where $\alpha \approx 1.83$ and $\beta \approx 3.13$ are numerical constants (Beloborodov et al., 2003). The high energy contribution $\delta \sigma_{1}$ in Eq. (2.5) contains the dimensionality of the array $d$ merely as a coefficient and is, in this sense, universal. On the contrary, the low energy contribution $\delta \sigma_{2}$ in Eq. (2.5) has a different functional form for different array dimensionalities (note that for the $3 \mathrm{~d}$ correction we have kept the temperature-dependent part only).

At high, $T>\Gamma$, temperatures the correction $\delta \sigma_{1}$, Eq. (2.5), grows logarithmically with decreasing temperature. Upon further lowering the temperature this correction saturates at $T \approx \Gamma$ and remains constant at $T<\Gamma$. Then the correction $\delta \sigma_{2}$ in Eq. (2.7) from the low energy scales, $\varepsilon \leq \Gamma$, where the coherent electron motion on the scales larger than the grain size $a$ dominates the physics, and which is similar to that derived for homogeneous disordered metals (Altshuler and Aronov, 1985) comes into play. In the low temperature regime it is this term that entirely determines the temperature dependence of the conductivity. At the same time, the contribution $\delta \sigma_{1}$, although being temperature independent, still exists in this regime and, as a matter of fact, can even be larger in magnitude than $\delta \sigma_{2}$. Equation (2.6) can be written for $T>\Gamma$ in the form Eq. (1.2) that has been observed in a number of experiments (Fuiimori et al., 1994; Gerber et al., 1997; Rotkina et al., 2005; Simon et al., 1987), (see, e.g. Fig. (5).

Rewriting the low energy contribution $\delta \sigma_{2}$, in terms of the effective diffusion coefficient $D_{\text {eff }}$ from
Eq. (2.3) one reproduces the Altshuler-Aronov corrections (Altshuler and Aronov, 1985). This reflects a universal character of a large scale behavior of a disordered system and we will show it rigorously in Sec. [I.D that indeed the granular metal model can be reduced to an effective disordered medium on distances much larger than the single grain size (i.e. coming from the low energy, $T<\Gamma$ excitations). The contribution $\delta \sigma_{1}$, which is dominated by the energies $\varepsilon>\Gamma$, is the consequence of and specific to the granularity - and does not exist in the homogeneously disordered metals.

Now we turn to quantum interference (weak localization) effects that exist also in the systems without any electron-electron interaction. In the metallic regime where the perturbation theory with respect to inverse tunneling conductance, $1 / \mathrm{g}$, the interaction and weak localization corrections can be considered independently in the leading order.

The weak localization correction is of a purely quantum origin: it stems from the quantum interference of electrons moving along the self-intersecting trajectories and is proportional to the return probability of an electron diffusing in a disordered medium. In one- or twodimensional conductors the probability of infinite times returns is unity and returning trajectories can be infinitely long. Thus a fully coherent electron propagation would lead to a divergent weak localization correction. Finite phase relaxation (dephasing) time disables long trajectories and limits the correction. Adapting here the concept of the effective disordered medium we can assume that the results of (Altshuler and Aronov, 1985) for homogeneously disordered metals apply to granular metals with the proper renormalization of the diffusion constant, we introduce the effective dephasing length $L_{\phi}=\sqrt{D_{\text {eff }} \tau_{\phi}} \sim a \sqrt{\Gamma \tau_{\phi}}$, where $\tau_{\phi}$ is the dephasing time, which dtermines the scale for the interference effects. At low temperatures the dephasing time $\tau_{\phi}$ is large and the decoherence length $L_{\phi}$ can exceed the size $a$ of a single grain, $L_{\phi}>a$. In this regime the relevant electron trajectories pass through many granules and quantum interference effects are similar to those in homogeneously disordered metals and contribute essentially to the conductivity. With the increasing temperature the decoherence length $L_{\phi}$ decreases and as soon as it drops to the grain size, $L_{\phi} \sim a$, the trajectories contributing to weak localizations traverse to only one neighbor and the weak localization correction is suppressed. Using Eq. (2.3) we write the last condition separating the domains of "relevance" and "irrelevance" of weak localization effects as $\tau_{\phi} \sim \Gamma^{-1}$. For $\tau_{\phi}>\Gamma^{-1}$ (or $L_{\phi}>a$ ) the quantum interference effects are important, while for $\tau_{\phi}<\Gamma^{-1}$ (or $\left.L_{\phi}<a\right)$ the corresponding correction drops rapidly with temperature.

The final result for the quantum interference corrections reads (Beloborodov et al., 2004c; Biagini et al., 2005a)

$$
\frac{\delta \sigma_{W L}}{\sigma_{0}}=-\frac{1}{4 \pi^{2} g} \ln \left(\tau_{\phi} \Gamma\right)
$$


for granular films, and

$$
\frac{\delta \sigma_{W L}}{\sigma_{0}}=-\frac{1}{2 \pi g}\left(\tau_{\phi} \Gamma\right)^{1 / 2},
$$

for granular wires. In both equations $\tau_{\phi}$ is the dephasing time. Within this time the wave function retains its coherence. The most common mechanism of dephasing, the electron-electron interactions gives for the dephasing time (Altshuler et al., 1982):

$$
\tau_{\phi}^{-1}= \begin{cases}\frac{T}{g} & d=2, \\ \left(\frac{T^{2} \delta}{g}\right)^{1 / 3} & d=1 .\end{cases}
$$

In this case the condition $\Gamma \approx \tau_{\phi}^{-1}$ defines (in $2 \mathrm{~d}$ case) yet another characteristic energy scale, $T^{*}=g^{2} \delta$, that marks the interval of relevance of weak localization effects.

The quantum interference correction $\delta \sigma_{W_{L}}$ are suppressed by applying even the relatively weak magnetic field; the dependence upon the magnetic field can serve as a test for identifying the weak localization effects. At sufficient fields thus the main temperature dependence of the conductivity will come from the electron-electron interaction effects, Eqs. (2.6) and (2.7).

Both, the electron-electron interactions and quantum interference effects decrease conductivity of a granular system at low temperatures similarly to the same effect in homogeneously disordered systems. The novel important feature is that the granularity restrains screening thus enhancing the role of Coulomb interaction: this is reflected by the contribution $\delta \sigma_{1}$ to conductivity which is specific to granular conductors but absent in the homogeneously disordered metals. As a result, in $3 d$ [and, to some extent, in $2 d$, see below], granular systems the Coulomb interactions can become a main driving force of a metal-insulator transition.

This question worth more detailed discussion. The "granular" contribution, $\delta \sigma_{1}$, comes from the short distances and is actually the renormalization of the tunneling conductance between the grains:

$$
g \rightarrow \tilde{g}=g-\frac{1}{2 \pi d} \ln \left[\frac{g E_{c}}{\max (T, \Gamma)}\right] .
$$

Using renormalization group methods one can show that Eq. (2.11) represnts in fact the solution of the renormalization group equation for the effective conductance $\tilde{g}$ rather than a merely perturbative correction. As such, it holds not only till the second term in the r. h. s. of Eq. (2.11) is much smaller than the first one but in a broader temperature region - as long as the renormalized conductance is large, $\tilde{g}>1$. It is important that the logarithm in Eq. (2.11) saturates at temperatures of the order of $\Gamma$. Then one sees from Eq. (2.11) that the renormalized conductance $\tilde{g}$ may remain large in the limit $T \rightarrow 0$ only provided the original (bare) conductance $g$ is larger than its critical value

$$
g_{c}=(1 / 2 \pi d) \ln \left(E_{c} / \delta\right) .
$$

If $g<g_{c}$ the effective conductance $\tilde{g}$ renormalizes down to zero at finite temperature. Of course, as soon as $\tilde{g}$ becomes of the order of unity, Eq. (2.11) is no longer valid, but it is generally accepted - in the spirit of the renormalization group approach - that the conductance flow to low values signals the electron localization (a recent exact solution for a model equivalent to a single grain connected to a metallic contact lends confidence to this conclusion (Lukvanov and Zamolodchikov, 2004)). The result (2.12) can be also obtained by the analysis of the stability of the insulating state that we discuss below.

The physical meaning of the critical conductance $g_{c}$ is most transparent for a $3 d$ system: in this case, there are no infrared divergencies and both the localization and Altshuler-Aronov corrections are not important even in the limit $T \rightarrow 0$. This implies that so long as $g>g_{c}$ the granular conductor remains metallic.

At $g<g_{c}$ the conductance renormalizes to low values at temperatures exceeding $\Gamma$ signaling thus the development of the Coulomb blockade. Upon the further temperature decrease the system resistance begins to grow exponentially at certain characteristic temperature $T_{c h}$ indicating the onset of the insulating behavior. The temperature $T_{c h}$ tends to zero in the limit $g \rightarrow g_{c}$. Thus, in $3 d$ the value $g_{c}$ marks the boundary between the insulating and metallic states at $T \rightarrow 0$.

The interpretation of the critical value $g_{c}$ is less straightforward in the case of granular films, since at $g>g_{c}$ the low temperature conductivity corrections due to interaction and localization effects diverge logarithmically. This means apparently, that the system turns an insulator without a sharp transition. Yet the notion of $g_{c}$ still makes sense as a mark distinguishing between the systems that are strong Coulomb insulators at low temperatures $\left(g<g_{c}\right)$ and those that are weak insulators $\left(g>g_{c}\right)$.

All the above results have been obtained within the model of a periodic granular array (cubic lattice) neglecting the dispersion of the tunneling conductances. In reality, the typical granular samples are disordered. It is then important to understand what effect the irregularities in the granule's arrangement may have. It is plausible and intuitive that the universal regime of low energies is hardly affected by the irregularities since all the physical characteristics in this regime can be expressed through the effective diffusion coefficient of the medium. At the same time, the physical results that are controlled by the local physics, in particular, the critical value $g_{c}$ are sensitive to the grain arrangements. In some cases the irregularities can be incorporated at almost no expense. For example, breaking some finite fractions of the junctions between the granules merely changes the average coordination number $z$, which appears as the $1 / 2 d$ factor in the expressions (2.11 2.12) for the critical conductance. This means that the dispersion in tunneling conductances is not expected to change noticeably, for example, the position of the metal-insulator transition (which may acquire the percolation character) and can 
be accounted for via replacing $2 d$ in Eqs. (2.11 2.12) by the effective coordination number $z_{\text {eff }}<z$.

In general the proper treatment of disorder in the grain arrangement may appear more tedious. Yet we do not expect that it can change the physics of the metallic state qualitatively. Irregularities of the grain displacements and of other quantities characterizing the system play much more important role in the insulating regime which we briefly review in the following subsection.

\section{Insulating regime}

We begin with a consideration of a periodic granular array which, in the regime of a weak coupling between the grains, is an exemplary Mott insulator at low temperatures. The electron transport is mediated by the electron hopping from grain to grain. However, leaving a neutral grain and entering its neighbor costs a considerable electrostatic energy, and the electron transport is blocked at low temperatures by the Coulomb gap in the electron excitation spectrum $\Delta_{M}$. At very small tunneling conductances this gap is simply the Coulomb charging energy of the grain, $\Delta_{M}=E_{c}$. Virtual electron tunneling to neighboring grains leads to a reduction of the Mott gap $\Delta_{M}$ and, in the limit of noticeable tunneling, it decays exponentially in $g$ (Beloborodov et al., 2005c) until it reaches the inverse escape rate from a single grain $\Gamma$. At $\Delta_{M} \sim \Gamma$ the system falls into a regime of weak Coulomb correlations and the insulator-metal transition occurs in $3 d$. Using the estimate $\Delta_{M} \sim \Gamma$ for estimating the transition point one arrives, within the logarithmic accuracy, at the same result for the critical conductance $g_{c}$ as that derived from a renormalization group considerations (see for details Section III.F).

The presence of the hard gap in the excitation spectrum leads to the activation dependence (Arrhenius law) of the conductivity on temperature:

$$
\sigma \sim e^{-\Delta_{M} / T}, \quad T \ll \Delta_{M} .
$$

Indeed, the finite temperature conductivity is due to the electrons and holes that are present in the system as real excitations. Their density is given by the Gibbs distribution that results in the exponential dependence of the conductivity, Eq. (2.13).

However, the activation behavior is usually not observed in real granular samples at low temperatures. Instead, the experimentally observed resistivity follows the law, Eq. (1.1), that resembles the Efros-Shklovskii law derived for doped semiconductors. The fact that the observed conductivity behavior cannot be explained in terms of the periodic model suggests that disorder plays the crucial role in formation the low temperature conductivity in the insulator state.

The stretched exponential Shklovskii-Efros-like conductivity behavior in granular conductors remained a challenging puzzle for a long time. Several explanations had been advanced, in particular, it was pro-

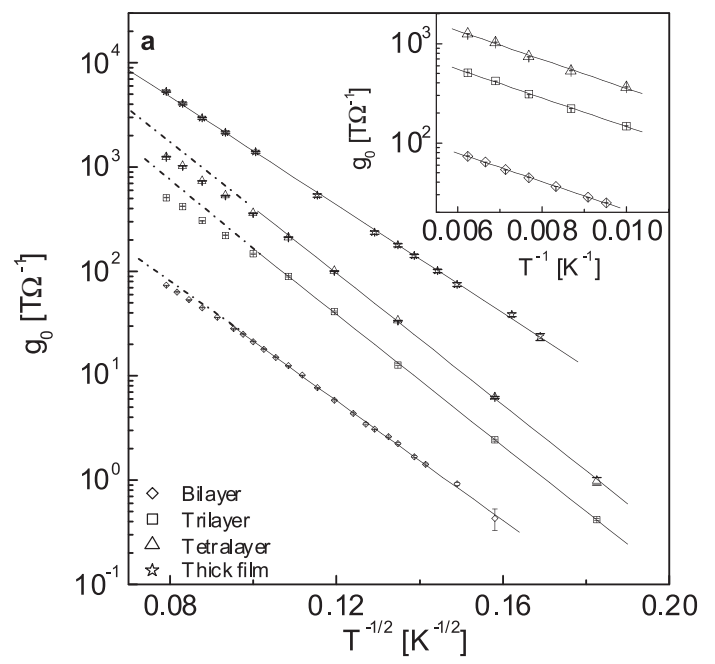

FIG. 6 Conductance $g_{0}$ vs inverse temperature $T^{-1 / 2}$ for periodic granular multilayer and thick-film shown in Fig. 2] (Tran et al., 2005). Inset: for the high-temperature range $g_{0}$ has been replotted as a function of $T^{-1}$, indicating Arrhenius behavior from $100-160 \mathrm{~K}$.

posed (Abeles et al., 1975), that the capacitance random variations resulting from grain size dispersion could provide the dependence, Eq. (1.1). However, as was pointed out in Refs. (Pollak and Adkins, 1992; Zhang and Shklovskii, 2004), the capacitance disorder can never lift the Coulomb blockade in a single grain completely and therefore cannot give rise to the finite density of states at the Fermi level. Furthermore, the stretched exponential dependence, Eq. (1.1), was recently observed in the periodic arrays of quantum dots Yakimov et al., 2003) and artificially manufactured metallic periodic granular systems (Tran et al., 2005), see Fig. 6] where the size of granules and the periodicity in the dots arrangement were controlled within a few percent accuracy. Either of those systems does not posses a noticeable capacitance disorder at all, yet the dependence (1.1) has been observed. These indicates that it should be electrostatic disorder unrelated to the grain size variations but caused most probably by charged defects in the insulating matrix/substrate that is responsible for lifting Coulomb blockade and formation the finite density of states near the Fermi level resulting in the dependence (1.1).

There is however another ingredient necessary for the variable-range-hopping type ( $\mathrm{VRH})$ conductivity to occur (apart the finite density of states at energies close to Fermi level): the finite, although exponentially decaying with the distance, probability, for tunneling to the spatially remote - and not only to the adjacent - states close to the Fermi level. Accordingly, the problem of the hopping transport in the granular conductors is two-fold and should contain: (i) explanation the origin of the finite density of states near the Fermi-level and the role of the Coulomb correlations in forming this density of states and (ii) constructing and quantitative description of the 
mechanism of tunneling over long distances through the dense array of metallic grains.

The behavior of the density of states, as we have mentioned above, can form due to the onsite random potential, which in its turn is induced by the carrier traps in the insulating matrix in the granular conductors. The traps with energies lower than the Fermi level are charged and induce the potential of the order of $e^{2} / \kappa r$ on the closest granule, where $\kappa$ is the dielectric constant of the insulator and $r$ is the distance from the granule to the trap. This compares with the Coulomb blockade energies due to charging metallic granules during the transport process. Such a mechanism was considered by Zhang and Shklovskii (2004). Speaking about the $2 d$ granular arrays and/or arrays of quantum dots, one can expect that the induced random potential originates also from imperfections and the charged defects in the substrate.

We model the electrostatic disorder via the random potential $V_{i}$, where $i$ is the grain index. Such a potential gives rise to a flat bare density of states at the Fermi level. In a complete analogy with semiconductors, the bare density of states should be suppressed by the longrang Coulomb interaction (Efros and Shklovskii, 1975; Shklovskii and Efros, 1988).

Next we have to consider the electron hopping over the distances well exceeding the average granule size in a dense granular array. This processes can be realized as tunneling via the virtual electron levels in a sequence of grains. The virtual electron tunneling through a single granule or a quantum dot (the so called co-tunneling) was first considered by Averin and Nazarov (1990) where two different mechanisms for a charge transport through a single quantum dot in the Coulomb blockade regime were identified. Namely, there are elastic and inelastic co-tunneling mechanisms. In the course of elastic cotunneling the charge is transferred via the tunneling of an electron through an intermediate virtual state in the dot such that the electron leaves the dot with the same energy as it came in. In the latter mechanism (inelastic co-tunneling) an electron that comes out of the dot has a different energy from that of the incoming one. After inelastic co-tunneling the electrons leaves behind in the granule the electron-hole excitation absorbing the in- and out- energy differences. Note that both these processes are realized via classically inaccessible intermediate states, i.e. both mechanisms occur in the form of the charge transfer via a virtual state. The inelastic cotunneling dominates at temperatures larger than $T_{1} \sim \sqrt{E_{c} \delta}$ (Averin and Nazarov, 1990).

These two co-tunneling mechanisms can be generalized to the case of the multiple co-tunneling through several grains. The tunneling probability should fall off exponentially with the distance (or the number of granules left behind $N$ ) (Beloborodov et al. 2005c; Feigel'man and Ioselevich, 2005; Tran et al., 2005) and this is equivalent to the exponentially decaying probability of the tunneling between the states near the Fermi surface in the theory of Mott-Efros-Shklovskii (Efros and Shklovskii, 1975; Shklovskii and Efros, 1988).

Thus the hopping processes in the amorphous semiconductors and granular materials are alike - up to the specific expressions for the localization lengths - and minimizing the hopping probability in the same manner as in the classic Efros-Shklovskii work, one ends up with the hopping conductivity $\sigma$ in a form

$$
\sigma \sim \exp \left[-\left(T_{0} / T\right)^{1 / 2}\right]
$$

where $T_{0}$ is a characteristic temperature depending on the particular microscopic characteristics. Explicit expressions for this temperature in different regimes are given in Section II.G. Eq. (2.14) explains the experimentally observed conductivity behavior in poorly conducting granular materials (see Eq. (1.1)).

Our discussion of the variable range hopping via virtual electron tunneling through many grains is based on the assumption that the hopping length $r^{*}$ exceeds the size of a single grain $a$. This length decays when the temperature increase and reaches the grain size at some characteristic temperature $\tilde{T}$. Then the VRH picture does not apply any longer, the hops occur between the adjacent granules only. Once the probability of a single jump is defined, the quantum effects can be neglected and one can use a classical approach. In particular, at temperatures $T \geq \tilde{T}$ one expects the conductivity to follow the simple Arrhenius law.

The classical approach to the transport in granular metals was developed in Refs. (Jha and Middleton, 2005; Middleton and Wingreen, 1993). One of the results of this study is the presence of the threshold voltage below which the conductivity is exactly zero at $T=0$. The existence of such a threshold voltage is a consequence of the classical approach where multiple cotunneling processes are not taken into account.

In order to match the results of the classical and hopping theories one has to generalize the approach of Middleton and Wingreen (1993) to include the multiple cotunneling processes. Development of such a theory, in our opinion, is an interesting and important task.

\section{B. Model and main theoretical tools}

From the theoretical point of view a granular conductor is an appealing exemplary system whose behavior is governed by a non-trivial interplay of electronelectron interactions, disorder and quantum fluctuations. The powerful approaches that allowed for recent breakthroughs in our understanding the physics of granular media are based on the effective field theories; in particular, the phase action technique appeared especially suitable. For the situations that fall outside its range of applicability, the appropriate diagrammatic techniques, generalizing those for the homogeneously disordered systems, can be developed. In this subsection we present 
in detail a model for description of granular metals and introduce both complimentary methods that serve as a foundation for quantitative description of granular conductors.

\section{Hamiltonian}

We model the granular system as an array of metallic particles connected via tunneling contacts. The Hamiltonian $\hat{H}$ describing a granular conductor has the form

$$
\hat{H}=\sum_{i} \hat{H}_{0, i}+\hat{H}_{I}+\hat{H}_{t}
$$

where $\hat{H}_{0, i}$ stands for the Hamiltonian of non-interacting electrons in a grain $i, \hat{H}_{c}$ represents the interactions, and $\hat{H}_{t}$ describes the electron tunneling between the grains. We now discuss each term in Eq. (2.15).

The $\hat{H}_{0, i}$ term describes the free electrons within the each grain in the presence of impurities

$$
\hat{H}_{0, i}=\int \hat{\psi}_{i}^{+}(\mathbf{r})\left(-\frac{\nabla^{2}}{2 m}+u_{i}(\mathbf{r})-\mu\right) \hat{\psi}_{i}(\mathbf{r}) d \mathbf{r},
$$

where $\hat{\psi}_{i}^{+}(\mathbf{r}), \hat{\psi}_{\mathbf{i}}(\mathbf{r})$ are the electron creation and annihilation operators, $\mu$ is the chemical potential and $u\left(\mathbf{r}_{i}\right)$ represents disorder responsible for the electron scattering inside the $i$-th grain. We adapt the Gaussian distribution for $u\left(\mathbf{r}_{i}\right)$ with pair correlations

$$
\left\langle u_{i}(\mathbf{r}) u_{j}\left(\mathbf{r}^{\prime}\right)\right\rangle=\frac{1}{2 \pi \nu \tau_{\mathrm{imp}}} \delta\left(\mathbf{r}-\mathbf{r}^{\prime}\right) \delta_{i j} .
$$

Throughout our review we assume that all the grains are in the diffusive limit, i.e. the electron mean free path $l$ within each grain is smaller than the grain size $a$. This assumption simplifies our calculations because it allows to avoid considering the electron scattering from the grain boundaries that becomes the main "disorder" mechanism in the case of ballistic grains. At the same time, most of the results obtained in the diffusive limit are expected to hold also for the ballistic grains with an irregular surface as long as the single grain diffusion coefficient $D_{0}$ does not enter the final result. This happens in the normal grains provided all relevant energies are smaller than the Thouless energy $E_{T h}$ of a single grain, Eq. (1.5). Actually, for typical grain sizes of the order $100 \AA$ the mean free path $l$ is comparable with the granule size $a$.

A single grain may be considered within the standard diagrammatic approach developed for disordered metals (Abrikosov et al., 1965). The main building block for the diagrams is a single electron Green function averaged over disorder and can be derived by the self consistent Born approximation (SCBA). The diagram shown in Fig. 7 represents the relevant contribution to the self energy:

$$
G_{0 \varepsilon}(\mathbf{p})=\left(i \varepsilon-\xi(\mathbf{p})+i \frac{\operatorname{sgn}(\varepsilon)}{2 \tau_{\mathrm{imp}}}\right)^{-1} .
$$

Another important block for the diagrammatic technique is the diffusion propagator (diffusion) which is just the impurity averaged particle-hole propagator. In bulk disordered metals it is given by

$$
D(\omega, \mathbf{q})=\frac{1}{D_{0} \mathbf{q}^{2}+|\omega|},
$$

where $D_{0}$ is the classical diffusion coefficient and $\omega$ is the Matsubara frequency.

In a grain, however, the term $\mathbf{q}^{2}$ has to be replaced by the Laplace operator with the proper boundary conditions (Aleiner et al., 2002; Efetov, 1983, 1997). This procedure leads to the quantization of the diffusion modes and to the appearance of the lowest excitation energy of the order of $D_{0} / a^{2}$ with $a$ being the grain size. This is just the Thouless energy $E_{T h}$ that has been defined in Eq. (1.5).

The Thouless energy of a nanoscale grain is large and this allows us to simplify calculations neglecting all non-zero space harmonics in the diffusion propagator, Eq. (2.19). Throughout our review we will assume that $E_{T h}$ is the largest energy scale associated with our system, and will use the zero dimensional approximation for the diffusion propagator

$$
D(\omega)=1 /|\omega|
$$

Now we turn to the description of the electron-electron interaction, the second term $\hat{H}_{I}$ in the Hamiltonian, Eq. (2.15). We begin with the consideration of the interaction effects in an isolated grain and then we will turn to the discussion of the intergranular terms that are important because of the long range character of the Coulomb interaction.

The most general form of the electron-electron interaction $\hat{H}_{I}$ in an isolated grain is

$$
\hat{H}_{I}^{(0)}=\frac{1}{2} \sum_{p, q, r, s} \mathcal{H}_{p q r s} \psi_{p, \alpha}^{+} \psi_{q, \beta}^{+} \psi_{r, \beta} \psi_{s, \alpha},
$$

where the subscripts $p, q, r, s$ stand for the states in the grains and $\alpha, \beta$ label electron spins.

The matrix $\mathcal{H}_{\text {pqrs }}$ is a complicated object, but in the low energy region of the parameters, not all of the matrix elements are of the same order. In the case of disordered grain that we consider, only the elements with the equal in pairs indices survive (Aleiner et al., 2002), all others are small in the parameter $1 / g_{0}$, where $g_{0}$ is the single grain conductance. Thus in the leading order in $1 / g_{0}$ the in-grain interaction term is (Aleiner et al., 2002)

$$
H_{I}^{(0)}=E_{c} \hat{n}^{2}+J_{S} \hat{\vec{S}}^{2}+\lambda \hat{T}^{\dagger} \hat{T}
$$

where $\hat{n}=\int \hat{\psi}^{+}(\mathbf{r}) \hat{\psi}_{i}(\mathbf{r}) d \mathbf{r}-N_{0}$ is the number of excessive (with respect to the electron number in the charge neutral state $N_{0}$ ) electrons in the grain, $\hat{\vec{S}}$ is the total spin of the grain and $\hat{T}^{\dagger}, \hat{T}$ are the Cooper pair creation 
and annihilation operators: $T=\sum_{p} \hat{\psi}_{p, \uparrow} \hat{\psi}_{p, \downarrow}$. The interaction strengths in these three channels are controlled by coupling constants $E_{c}, J_{S}, \lambda$ respectively. The coupling constants in the spin and BCS channels, $J_{S}$ and $\lambda$, cannot be large and are, at most, of the order of $\delta$. At the same time the charging energy $E_{c}$ is usually much larger than $\delta$. For this reason, in the absence of the superconductivity, the most interesting and noticeable effects come from the Coulomb correlations.

The above considerations of the interactions in a single grain can be easily generalized to the case of a granular array. It is clear that the bare interactions in the spin and Cooper pair channels are short-range and thus they have to be diagonal in the granular indices. At the same time the Coulomb interaction is long-range and it's of-diagonal components cannot be neglected. Thus, we arrive at the following Hamiltonian that describes the Coulomb correlations

$$
\hat{H}_{c}=\frac{e^{2}}{2} \sum_{i j} \hat{n}_{i} C_{i j}^{-1} \hat{n}_{j}
$$

where $C_{i j}^{-1}$ is the capacitance matrix which can be found by solving a classical electrostatic problem of metallic particles embedded into the insulating matrix. Note that since metallic grains have infinite dielectric constant, the effective dielectric constant of the whole sample can be considerably larger than the dielectric constant of its insulating component. Thus the effective single grain charging energy can be much less than the electrostatic energy of a single grain in a vacuum.

The Hamiltonian $\hat{H}_{c}$ describing the long range part of the Coulomb interaction, (2.23), has been derived for granular superconductors in the early work (Efetov, 1980). As far as the normal grains are concerned, the proper derivation is given in the review (Aleiner et al., 2002). The term $\hat{H}_{c}$, Eq. (2.23), commutes with the free part $\hat{H}_{0, i}$, Eq. 2.16) and therefore does not describe dynamics in a single insulated granule. For the macroscopic transport to occur the electron trasfer from grain to grain has to be switched on, and we turn now to the third term $\hat{H}_{t}$ in Eq. (2.15) describing the electron tunneling between the neighboring grains. We write this term in the form

$$
\hat{H}_{t}=\sum_{i, j ; p, q} t_{i j ; p q} \hat{\psi}_{p i}^{+} \hat{\psi}_{q j},
$$

where the summation is performed over the states $p$, $q$ of each grain and over the neighboring grains $i$ and j. Abrikosov, 1988; Ambegaokar and Baratoff, 1963; Cohen et al., 1962). It is assumed that the linear size of the inter-grain contact area well exceeds atomic distances, i.e. there is a large number of conducting channels between the grains. At the same time, the magnitude of the potential barrier between the grains can be large, this can easily be achieved experimentally by the process of oxidation. Therefore, the tunneling conductance $g$, which is roughly proportional to the area of the contact and to the square of the tunneling matrix element $t_{i j}$, can be set both larger and smaller than unity.

While the dependence of the tunneling elements $t_{i j ; p q}$ on the state numbers $p, q$ is not important for transport through the conventional point contacts and is usually neglected (Abrikosov, 1988; Ambegaokar and Baratoff, 1963; Cohen et al., 1962), it may become relevant for granular arrays. Namely, the importance of this dependence is related to whether the electron motion in the grains is chaotic or can be viewed as an integrable billiard. In order to clarify this point we write the matrix elements $t_{i j ; p q}$ as

$$
t_{i j ; p q}=\int t_{i j}\left(\mathbf{s}_{i j}\right) \phi_{p}^{*}\left(\mathbf{s}_{i j}\right) \phi_{q}\left(\mathbf{s}_{i j}\right) d \mathbf{s}_{i j},
$$

where $\mathbf{s}_{i j}$ is the coordinate on the junction between the grains $i$ and $j$ and $\phi_{p}\left(\mathbf{r}_{i}\right)$ are the wave functions. The integration over $s_{i j}$ in Eq. 2.25) extends over the area of the junction.

If the grains do not contain any disorder and have a perfect shape (e.g. cubes or spheres), the array is a completely periodic system and according to the Bloch theorem the total resistivity is zero. The presence of the internal disorder or irregularities of the shape of the grains change the situation giving rise to a finite resistivity. In the latter case one may employ the random matrix theory (RMT) (Alhassid, 2000; Beenakker, 1997; Mehta, 1991) for an isolated single grain or, which is equivalent, the zero dimensional $\sigma$-model (Efetov, 1997). It is well known (Mehta, 1991) that this theory, when applied to the eigenfunctions leads to a Gaussian distribution $W\left\{\left|\phi_{p}(\mathbf{r})\right|\right\}$ of their amplitudes

$$
W\left\{\left|\phi_{p}(\mathbf{r})\right|\right\}=\exp \left(-\left|\phi_{p}(\mathbf{r})\right|^{2} V\right),
$$

where $V$ is the volume of the grain. Further, the Gaussian distribution of the amplitudes of the wave functions $\phi_{p}\left(\mathbf{r}_{i}\right)$ and the fact that their correlations decay rapidly with the increasing both spatial distance and the energy levels spacing (the characteristic scales are the wavelength and the mean level spacing, respectively) leads to a Gaussian distribution of the matrix elements $t_{i j ; p q}$.

A simple transformation brings these correlators to:

$$
\begin{aligned}
\left\langle t_{p_{1} q_{1}} t_{p_{2} q_{2}}\right\rangle & =\frac{g_{i j} \delta_{i} \delta_{j}}{2 \pi}\left(\delta_{p_{1} p_{2}} \delta_{q_{1} q_{2}}+\delta_{p_{1} q_{2}} \delta_{p_{2} q_{1}}\right), \\
\left\langle t_{p q}\right\rangle & =0 .
\end{aligned}
$$

In Eq. (2.27) the matrix elements $t_{p q}$ are taken for the same contact between the grains $i$ and $j$, otherwise the correlations vanish. As we will see, the constant $g_{i j}$ is nothing but the tunneling conductance of the contact.

Equation (2.27) is written for time reversal invariant systems (orthogonal ensemble). This, in particular, means that there is no magnetic field and/or and no magnetic impurities present and therefore all eigenfunctions of an insulated grain can be chosen real. In the limit of comparatively strong magnetic fields, one arrives at the 


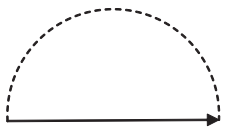

a)

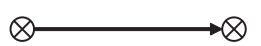

b)
FIG. 7 Self energy of the election Green function averaged over the impurity potential inside the grains and over tunnelling elements between the grains. Averaging over the impurity potential is represented by the dotted line (a) while tunnelling elements are represented by crossed circles (b).

unitary ensemble and the first term in the correlation function of the matrix elements in Eq. (2.27) vanishes. However, for the metallic grains of the size of the order of $5-10 \mathrm{~nm}$ the characteristic magnetic field that require the description in terms of the unitary ensemble is of the order of several Tesla. Thus in the subsequent discussion we will be using Eq. (2.27), assuming that the applied magnetic fields are not that high.

To conclude this part, we have reformulated the initial theory with impurities and regular tunneling matrix elements in terms of the model with random tunneling elements. This equivalence holds for the zero dimensional grains, or in other words, for the situations when all the characteristic energies are smaller than the Thouless energy $E_{T h}$, Eq. (1.5). Working with the random tunneling elements turns out the more convenient approach.

The model introduced in this subsection, Eqs. 2.15 2.16] 2.17 2.23 2.24] 2.27) describes a strongly correlated electronic disordered system which cannot be solved exactly. Below we introduce two complimentary approaches to explore this problem in different regimes. One of these approaches - the diagrammatic technique is especially useful provided the perturbative expansion with respect to one of the relevant parameters is possible. The second method is based on the gauge transformation which allows to get rid of the explicit Coulomb interaction term in the Hamiltonian at the expense of an appearing phase field. As we will show below, this approach offers a very powerful tool in its domain of applicability.

\section{Diagrammatic technique for granular metals}

There are two routes to construct diagrammatic technique for granular metallic systems. First, one can work in the basis of the exact single grain eigenfunctions and use the distribution of the tunneling elements (2.27) for the description of the scattering between the different states of the neighboring grains. In this case the impurities inside each grain are treated "exactly," and, by definition, the diagrams that represent impurity scattering within each grain do not appear in this representation. The alternative approach is, in essence, equivalent to the conventional cross technique: one begins with the momentum representation and then carries out the standard a)

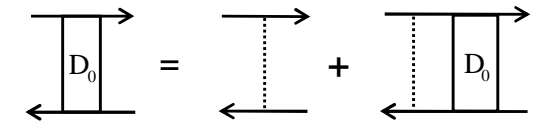

b)
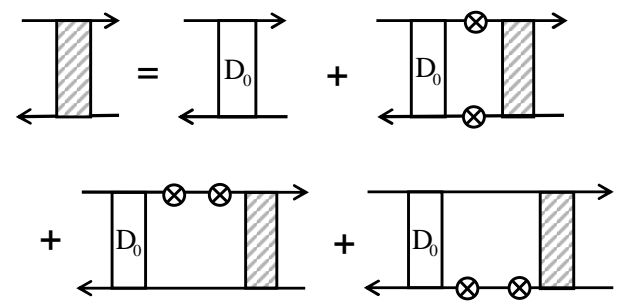

FIG. 8 Diagrams represent the Dyson equation for a single grain diffusion propagator Eq. 2.20 (a) and for the whole granular system Eq. 2.29 (b). Dotted lines represent the impurity scattering while crossed circles stay for intergranular tunnelling elements.

averaging over impurities within the each grain. The tunneling between the grains can be then viewed as an intergranular scattering. The corresponding matrix elements, as in the first method can, be viewed as Gaussian random variables. We prefer to follow the latter approach since it is straightforwardly related to the standard diagrammatic technique for homogeneously disordered metals making it easier to make comparisons to well known situations in disordered metals when possible.

Following the guidelines of Abrikosov et al. (1965) we construct the action expansions with respect to both types of disorder: potential disorder within the each grain $u(\mathbf{r})$ and the intergranular scattering matrix elements $t_{p q}$. Shown in Fig. 7 are the lowest order diagrams representing both contributions to the self energy of the single electron Green function in the granular metals obtained by averaging over $u(\mathbf{r})$ and $t_{p q}$. The diagram a) describes the potential scattering within a single grain, while the diagram b) is due to the intergranular scattering. Both processes result in a similar contribution proportional to $\operatorname{sgn} \omega$ to the electron self-energy. This shows, that on the level of the single electron Green function; the intergranular scattering results merely in the renormalization of the relaxation time $\tau$

$$
\tau^{-1}=\tau_{0}^{-1}+2 \Gamma d
$$

where $\tau_{0}$ is the electron mean free time in a single grain.

The next step is to consider the diffusion motion of electron through the granular metal. The diffusion motion inside a single grain is given by the usual ladder diagram that results in the diffusion propagator $D(\omega)$, Eq. (2.20). The tunneling between the grains does not change the selection rules for the diagrams. Typical diagrams are shown in Fig. 8 b). The diagrams are generated by connection the tunneling vertices, and only the diagrams without the intersection are to be kept. This is similar to what one has in the standard impurities tech- 
nique (Abrikosov et al., 1965). In order to derive the expression for the total diffusion propagator one should sum up the ladder diagrams shown in Fig. 8 b). For the periodic array of the grains we arrive at the following formula for the diffusion propagator $D(\omega, \mathbf{q})$

$$
D(\omega, \mathbf{q})=\tau^{-1}\left(|\omega|+\Gamma \lambda_{\mathbf{q}}\right)^{-1}
$$

where

$$
\lambda_{\mathbf{q}}=2 \sum_{\mathbf{a}}(1-\cos \mathbf{q} \mathbf{a}),
$$

$\mathbf{a}$ is the (super) lattice vectors, and $\mathbf{q}$ is the quasimomentum. In Eq. (2.29) only the zero space harmonics of the diffusion motion in the grain is taken into account. This approximation is valid in the limit

$$
\Gamma \ll E_{T h}
$$

where the energies $\Gamma$ and $E_{T h}$ are given by Eqs. (2.2) and (1.5), respectively. In the limit of small quasimomenta, $q \ll a^{-1}$, we have $\lambda_{q} \rightarrow a^{2} q^{2}$, such that the propagator (2.29) describes the diffusion motion on the scales much larger than the size of a single grain $a$ with an effective diffusion coefficient

$$
D_{e f f}=a^{2} \Gamma .
$$

The other necessary blocks of the diagrammatic technique can be constructed analogously to the diffusion propagator.

\section{Coulomb interaction and gauge transformation}

Taking an alternative route, one can eliminate the explicit Coulomb term in the model for the granular metals, Eqs. (2.15] 2.16] 2.17 2.23 2.24 2.27), by introducing a new phase field via a gauge transformation. This approach proves to be especially useful in the regime of strong Coulomb correlations, where the diagrammatic technique developed in the previous subsection may break down.

To introduce this approach it is convenient to adapt the formalism of the functional integration that allows replacing the calculations with the Hamiltonian $\hat{H}$, Eq. (2.15), by computation of a functional integral over classical fermion fields $\psi_{i}(X)$ and their complex conjugate $\psi_{i}^{*}(X)$, where $X=(\mathbf{r}, \tau)$. These fields must satisfy the fermionic antiperiodicity condition

$$
\psi_{i}(\tau)=-\psi_{i}(\tau+\beta)
$$

where $\beta=1 / T$ is the inverse temperature. We define the field $\psi_{i}(X)$ such that it is different from zero only in the $i$ th grain.

The Lagrangian $L[\psi]$ entering the functional integral for the partition function $Z$

$$
Z=\int \exp \left(-\int_{0}^{\beta} L[\psi] d \tau\right) D \psi
$$

takes the form

$$
L[\psi]=\sum_{i} L_{0 i}[\psi]+L_{t}[\psi]+L_{c}[\psi]
$$

where

$$
\begin{gathered}
L_{0 i}[\psi]=\int \psi_{i}^{*}(X)\left(\frac{\partial}{\partial \tau}-\frac{\nabla^{2}}{2 m}+u(\mathbf{r})-\mu\right) \psi_{i}(X) d \mathbf{r} \\
L_{t}[\psi]=\sum_{i, j ; p, q} t_{i j ; p q} \psi_{p i}^{*}(\tau) \psi_{q j}(\tau)
\end{gathered}
$$

and

$$
L_{c}[\psi]=\frac{e^{2}}{2} \sum_{i j} n_{i}(\tau) C_{i j}^{-1} n_{j}(\tau)
$$

where $n_{i}(\tau)=\int \psi_{i}^{*}(X) \psi_{i}(X) d \mathbf{r}$ is the density field of the grain $i$.

The Coulomb interaction $L_{c}[\psi]$, Eq. (2.38), can be decoupled using a Gaussian integration over the auxiliary field $\bar{V}_{i}(\tau)$ (Efetov and Tschersich, 2003)

$$
\begin{aligned}
& \exp \left[-\frac{e^{2}}{2} \sum_{i j} \int_{0}^{\beta} n_{i}(\tau) C_{i j}^{-1} n_{j}(\tau) d \tau\right] \\
= & \int \exp \left[i \sum_{i} \int_{0}^{\beta} \bar{V}_{i}(\tau) \psi_{i}^{+}(X) \psi_{i}(X) d X\right] \\
& \times Z_{\bar{V}}^{-1} \exp [-S(\bar{V})] d \bar{V}_{i}
\end{aligned}
$$

where $\bar{V}_{i}(\tau)$ is the bosonic field obeying the boundary condition $\bar{V}_{i}(\tau)=\bar{V}_{i}(\tau+\beta)$ and $Z_{\bar{V}}$ is the partition function,

$$
Z_{\bar{V}}=\int \exp [-S(\bar{V})] d \bar{V}_{i}
$$

The action $S(\bar{V})$ has the following form

$$
S[\bar{V}]=\frac{1}{2 e^{2}} \int_{0}^{\beta} d \tau \sum_{i j} \bar{V}_{i}(\tau) C_{i j} \bar{V}_{j}(\tau)
$$

We see from Eqs. 2.32 - 2.39) that the Lagrangian becomes quadratic in fields $\psi$ after the decoupling, Eq. (2.39). Instead of dealing with the Coulomb interaction, $L_{c}[\psi]$, Eq. (2.38), one should consider now an effective Lagrangian $L_{0 i}^{\text {eff }}[\psi, \bar{V}]$ for the grain $i$ :

$$
L_{0 i}^{e f f}[\psi, \bar{V}]=L_{0 i}[\psi]-i \int_{0}^{\beta} \bar{V}_{i}(\tau) \psi_{i}^{+}(\tau) \psi_{i}(\tau) d \tau
$$

The effective action $L_{0 i}^{e f f}[\psi, \bar{V}]$ is now expressed in terms of electron motion in a granular matter in the presence of the fluctuating potential $\bar{V}_{i}(\tau)$ of the grains. 
We remove the field $\bar{V}_{i}(\tau)$ from the Lagrangian $L_{0 i}^{\text {eff }}[\psi, \bar{V}]$, Eq. (2.41), using a gauge transformation of the fermionic fields

$$
\psi_{i}(\mathbf{r}, \tau) \rightarrow e^{-i \varphi_{i}(\tau)} \psi_{i}(\mathbf{r}, \tau), \quad \dot{\varphi}_{i}(\tau)=\bar{V}_{i}(\tau),
$$

where the phases $\varphi_{i}(\tau)$ depend on the imaginary time $\tau$ but not on the coordinates inside the grains. This is a consequence of the form of the Coulomb interaction, Eqs. (2.23 2.38). Since the action of an isolated grain is gauge invariant, the phases $\varphi_{i}(\tau)$ enter the whole Lagrangian of the system only through the tunneling matrix elements

$$
t_{i j} \rightarrow t_{i j} e^{i \varphi_{i j}(\tau)}
$$

where $\varphi_{i j}(\tau)=\varphi_{i}(\tau)-\varphi_{j}(\tau)$ is the phase difference of the $i$-th and $j$-th grains.

At the first glance, the transformation, Eq. (2.42), have removed completely the potentials $\bar{V}(\tau)$ from the effective Lagrangian $L_{0 i}^{\text {eff }}[\psi, \bar{V}]$, Eq. (2.41). In fact, this is not the case since the gauge transformation as defined in Eq. (2.42), violates the antiperiodicity condition, Eq. (2.33) and the resulting effective Lagrangian for the single grain changes. In order to preserve the boundary condition, Eq. (2.33), the certain constraints should be imposed on the phases $\varphi_{i}(\tau)$ and potentials $\bar{V}_{i}(\tau)$. Namely, the phases should obey the condition

$$
\varphi_{i}(\tau)=\varphi_{i}(\tau+\beta)+2 \pi k_{i}
$$

which leads to the following constraint for the potential $\bar{V}_{i}(\tau)$

$$
\int_{0}^{\beta} \bar{V}_{i}(\tau) d \tau=2 \pi k_{i}
$$

where $k_{i}=0, \pm 1, \pm 2, \pm 3 \ldots$.

The constraint on the phases $\varphi_{i}(\tau)$, Eq. (2.44), can be reformulated via introducing a function $\phi_{i}(\tau)$ assuming arbitrary real values from $-\infty$ to $\infty$ and obeying a periodicity condition

$$
\phi(\tau)=\phi(\tau+\beta) .
$$

With the aid of this function the phase $\varphi_{i}(\tau)$ can be written in the form:

$$
\varphi_{i}(\tau)=\phi_{i}(\tau)+2 \pi T k_{i} \tau
$$

which satisfies Eq. (2.44). The potential $\bar{V}_{i}(\tau)$, in its turn, is taken in a form

$$
\bar{V}_{i}(\tau)=\rho_{i}+\tilde{V}_{i}(\tau)
$$

where the static variable $\rho_{i}$ varies in the interval

$$
-\pi T<\rho_{i}<\pi T
$$

and the dynamic variable $\tilde{V}_{i}(\tau)$ satisfies the constraint (2.45). Note that the static part of the potential $\rho_{i}$ cannot be gauged out, contrary to dynamic contribution,
$\tilde{V}_{i}(\tau)$, and the effective Lagrangian assumes the form $L_{0 i}^{e f f}[\psi, \rho]$. In the limit of not very low temperatures,

$$
T \gg \delta
$$

the static potential $\rho_{i}$ drops out from the fermionic Green functions and does not influence the system behavior. This can be understood by noticing that at moderately high temperatures 2.50) the discreteness of the levels in the grains is not important. Then, in the Green functions, one may replace the variable $\varepsilon_{p}-\mu \rightarrow \xi$, where $\varepsilon_{p}$ are eigenenergies and $\xi$ is a continuous variable varying from $-\infty$ to $\infty$. Integrating over $\xi$ one may shift the contour of the integration into the complex plane and remove $\rho_{i}$ provided it obeys the inequality (2.49). More details can be found in Efetov and Tschersich (2003). Note that the variable $\rho_{i}$ cannot be neglected in the Lagrangian $L_{0 i}^{e f f}[\psi, \rho]$ in the limit of very low temperatures.

An integer $k_{i}$ in Eqs. (2.44 2.45 2.47) represents an extra degree of freedom related to the charge quantization and is usually called the "winding number". The physical meaning of the winding numbers becomes especially clear in the insulating regime where they simply represent static classical electron charges.

Thus, at not very low temperatures, 2.50), one can write the partition function $Z$ in the form

$$
Z=\int \exp \left[-\int\left(L_{0}[\psi]+L_{1}[\psi, \varphi]+L_{2}[\varphi]\right) d \tau\right] D \psi D \varphi
$$

where

$$
L_{0}[\psi]=\sum_{i} L_{0 i}[\psi]
$$

with $L_{0 i}[\psi]$ from Eq. (2.36). The tunneling term $L_{1}[\psi, \varphi]$ is

$$
L_{1}[\psi, \varphi]=\sum_{i j} \int_{0}^{\beta} d \tau t_{i j ; p q} \psi_{i p}^{*}(\tau) \psi_{j q}(\tau) \exp \left[i \varphi_{i j}(\tau)\right]
$$

and the term $L_{2}[\varphi]$ describing the charging effects reads

$$
L_{2}[\varphi]=\sum_{i j} \frac{C_{i j}}{2 e^{2}} \frac{d \varphi_{i}(\tau)}{d \tau} \frac{d \varphi_{j}(\tau)}{d \tau} .
$$

One should integrate in Eq. 2.51) over the anticommuting variables $\psi$ with the antiperiodicity condition Eq. 2.33). The integration over $\varphi$ includes the integration over the variable $\phi_{i}$ (see Eq. (2.47) ) and summation over $k_{i}$. Equations (2.51] 2.54 2.46 2.47) completely specify the model that will be studied in the subsequent sections.

\section{Ambegaokar-Eckern-Schön functional}

The partition function $Z$, Eqs. (2.51-2.54), can be further simplified by integration over the fermion fields $\psi$. 
This can hardly be done exactly but one can use a cumulant expansion in the tunneling term $L_{1}[\psi, \varphi]$, Eq. (2.53). Of course, even in the absence of the tunneling the random potential in $L_{0}[\psi]$, Eqs. (2.52 2.36), can make the problem highly non-trivial. Yet in the limit of not very low temperatures, Eq. (2.50), the problem can be simplified because of the possibility of neglecting interference effects and considering the disorder within the SCBA taking Green functions from Eq. (2.18). We perform the cumulant expansion in the tunneling term $S_{1}[\psi, \varphi]$ up to the lowest non-vanishing second order, integrating over fermionic degrees of freedom and averaging over the tunneling matrix elements with the help of Eq. (2.27).

The resulting action, originally derived in Ambegaokar et al. (1982) for a system of two weakly coupling superconductors, contains only the phases $\varphi_{i}(\tau)$ but no fermionic degrees of freedom left. Then for the granular metals the partition function $Z$ can be written as

$$
Z=\int \exp (-S) D \varphi, \quad S=S_{c}+S_{t}
$$

where $S_{c}$ describes the charging energy

$$
S_{c}=\frac{1}{2 e^{2}} \sum_{i j} \int_{0}^{\beta} d \tau C_{i j} \frac{d \varphi_{i}(\tau)}{d \tau} \frac{d \varphi_{j}(\tau)}{d \tau}
$$

and $S_{t}$ stands for tunneling between the grains

$$
S_{t}=\pi g \sum_{\langle i j\rangle} \int_{0}^{\beta} d \tau d \tau^{\prime} \alpha\left(\tau-\tau^{\prime}\right) \sin ^{2}\left[\frac{\varphi_{i j}(\tau)-\varphi_{i j}\left(\tau^{\prime}\right)}{2}\right]
$$

The function $\alpha\left(\tau-\tau^{\prime}\right)$ in Eq. (2.57) has the form

$$
\alpha\left(\tau-\tau^{\prime}\right)=T^{2} \operatorname{Re} \sin ^{-2}\left[\pi T\left(\tau-\tau^{\prime}+i \eta\right)\right] .
$$

where $\eta \rightarrow+0$ and one should take the real part in Eq. (2.58).

Despite the fact that the above functional was obtained via an expansion in the tunneling term $S_{1}[\psi, \varphi]$, its validity is not limited by the insulating regime, $g \ll 1$, only. The functional can be used in the metallic regime at temperatures $T \gg \Gamma$, where $\Gamma$ is given by Eq. (2.2).

Of course, the phase action looses some information about the original model, Eqs. (2.34 - 2.38), and its applicability in each particular case has to be carefully analyzed. In general, the functional $S$, Eqs. (2.55-2.58) does not apply in cases where the coherent diffusive motion of an electron on the scale of many grains is important. For example, the weak localization correction cannot be obtained within this approach.

Moreover, one has to be careful in using this approach even in the weak coupling regime, $g \ll 1$. For example, the hopping conductivity in the low temperature elastic regime is also beyond the accuracy of the phase action, Eqs. 2.55 2.58), since it requires consideration of the elastic multiple co-tunneling processes which the phase action misses.
Yet the phase functional approach is extremely powerful for many applications. For example, it enables obtaining non-perturbative results for the conductivity in the metallic regime at temperatures $T \gg \Gamma$.

\section{Metallic properties of granular arrays at not very low temperatures}

In this section, we will derive the logarithmic correction to the conductivity, Eq. (2.6), in the temperature regime $T \gg \Gamma$. While this correction has the same origin as the Althsuler-Aronov (AA) result derived for the homogeneously disordered metals (Altshuler and Aronov, 1985; Lee and Ramakrishnan, 1985), its form is specific to granular metals because the AA correction. In contrast to Eq. (2.6), AA corrections are sensitive to the sample dimensionally and exhibit the logarithmic behavior only in two dimensions.

The logarithmic correction, Eq. (2.6), as we will show below, can be obtained as a result of the renormalization of the tunneling coupling $g$ due to the Coulomb correlations. It comes from the short distances, and this explains its insensitivity to the sample dimensionally. At temperatures smaller than $\Gamma$ the coherent electron motion on the scales of many grains becomes important, and the conductivity correction acquires the form similar to that of the homogeneously disordered metals.

For the sake of simplicity we will consider a periodic system assuming that the granular array forms a cubic lattice. This implies that all the grain sizes and intergranular conductances are equal to each other. At the end of this section we will discuss the applicability of the result (2.6) to systems containing irregularities. We will start with perturbation theory in $1 / g$, then generalize our derivation of Eq. (2.6) using the renormalization group approach.

\section{Perturbation theory}

In the limit of large tunneling conductances $g \gg 1$ the tunneling term $S_{t}$, Eq. (2.57), suppresses large fluctuations of the phase $\varphi(\tau)$. In this regime the tunneling term $S_{t}$ can be expanded in phases $\varphi_{i j}(\tau)$ because they are small. The charge quantization effects in this regime are not pronounced allowing to neglect all non-zero winding numbers $k_{i}$. At the same time, the phase fluctuations can change considerably the classical result, Eq. (2.1).

The quadratic in $\varphi_{i}(\tau)$ part of the action $S$ in Eq. (2.55 - 2.57) will serve as the bare action for the perturbation theory. Keeping terms of the second order in $\varphi_{i}(\tau)$ in Eqs. (2.55 - 2.57) and performing the Fourier transformation in both the coordinates of the grains and the imaginary time we reduce the action $S$ in Eq. (2.55) to the form

$$
S_{0}=T \sum_{\mathbf{q}, n} \varphi_{\mathbf{q}, n} G_{\mathbf{q}, n}^{-1} \varphi_{-\mathbf{q},-n}
$$




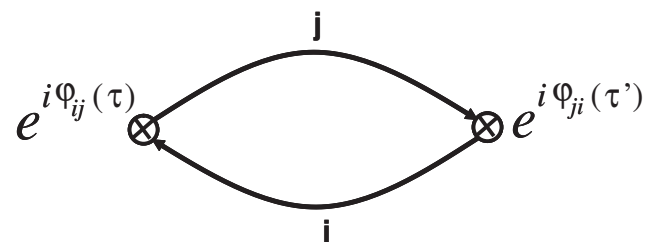

FIG. 9 This diagram represents the conductivity in the leading order in $1 / \mathrm{g}$. The crossed circles represent the tunnelling matrix elements $t_{k k^{\prime}}^{i j} e^{i \varphi_{i j}(\tau)}$ where phase factors appear from the gauge transformation.

where

$$
G_{\mathbf{q} n}^{-1}=\omega_{n}^{2} / 4 E(\mathbf{q})+2 g\left|\omega_{n}\right| \lambda_{\mathbf{q}} .
$$

where $\lambda_{\mathbf{q}}$ is given by Eq. (2.30). Here $\mathbf{q}$ are the quasi-momenta of a periodic array, $E(\mathbf{q})$ is the Fourier transform of the charging energy related to the Fourier transform of the capacitance matrix $C(\mathbf{q})$ as $E(\mathbf{q})=$ $e^{2} / 2 C(\mathbf{q})$. The conductivity $\sigma(\omega)$ of the array of the grains can be obtained from the current-current correlation function via the standard Kubo formula. The conductivity in the leading order in $1 / g$ is shown in Fig. 9 Its analytical expression is given by (Efetov and Tschersich, 2003)

$\sigma(\omega)=\frac{2 \pi e^{2} g T^{2} i a^{2-d}}{\omega} \int_{0}^{\beta} d \tau \frac{1-e^{i \Omega \tau}}{\sin ^{2}(\pi T \tau)} \exp \left(-\tilde{G}_{\mathbf{a}}(\tau)\right)$,

where the analytic continuation to the real frequency is assumed as $\Omega \rightarrow-i \omega$ and the function $\tilde{G}_{\mathbf{a}}(\tau)$ is

$$
\tilde{G}_{\mathbf{a}}(\tau)=4 T a^{d} \sum_{\omega_{n}>0} \int \frac{d^{d} \mathbf{q}}{(2 \pi)^{d}} G_{\mathbf{q} n} \sin ^{2} \frac{\mathbf{q} \mathbf{a}}{2} \sin ^{2} \frac{\omega_{n} \tau}{2} .
$$

where $d$ is the dimensionality of the array. The factor $\exp \left(-\tilde{G}_{\mathbf{a}}(\tau)\right)$ in Eq. (2.61) is responsible for the interaction effects and appears as a result of the averaging of the phase exponents $e^{i \phi(\tau)}$ emerging after the gauge transformation, Eq. (2.42). One can see from Eqs. 2.60 2.62 ) that the function $\tilde{G}_{\mathbf{a}}(\tau)$ behaves as a logarithm $\ln \left(g E_{c} \tau\right)$ at the values of $\tau$ of the order $1 / T$ that are essential for calculation. With the logarithmic accuracy one can neglect the $\omega_{n}^{2}$ term in $G_{\mathbf{q}, n}^{-1}$, Eq. 2.60), and reduce Eq. (2.62) to

$$
\tilde{G}_{\mathbf{a}}(\tau)=\frac{T}{d g} \sum_{\omega_{n}>0}^{\omega_{c}} \frac{1-\cos \left(\omega_{n} \tau\right)}{\omega_{n}} .
$$

In Eq. (2.63) one should sum over positive Matsubara frequencies up to the cutoff $\omega_{c} \sim g E_{c}$. For larger frequencies the first term in Eq. (2.60) is no longer small and there is no logarithmic contribution from these large frequencies. Equation (2.63) shows a remarkable independence of the result on the structure of the lattice. What is also important, there are no "infrared" divergencies in the integral over $\mathbf{q}$ in any dimensionality including $2 d$ and $1 d$.
With the logarithmic accuracy, one can replace $\tau$ by $1 / T$ in the function $\tilde{G}_{\mathbf{a}}(\tau)$ and calculate the remaining integral over $\tau$ in Eq. (2.61) ignoring the dependence of the function $\tilde{G}_{\mathbf{a}}$ on $\tau$. Taking the limit $\omega \rightarrow 0$ we obtain the result (2.6) in the temperature interval $T \gg \Gamma$ :

$$
\sigma=\sigma_{0}\left(1-\frac{1}{2 \pi d g} \ln \left[\frac{g E_{c}}{T}\right]\right) .
$$

It was shown in Efetov and Tschersich (2003) that the terms of the order $(1 / g)^{2} \ln ^{2}\left(g E_{c} / T\right)$ are cancelled out in the expansion of the conductivity correction, which means that the accuracy of Eq. 2.64) exceeds the accuracy of the first order correction. Furthermore, this cancellation is not accidental and the result (2.64) turns out to be applicable even at temperatures at which the conductivity correction becomes of the same order as $\sigma_{0}$ itself. This fact is explained in the next subsection where the temperature dependence of the conductivity is considered within the RG scheme.

\section{Renormalization Group}

In order to sum up all the logarithmic corrections to the conductivity we use RG scheme suggested for a one-dimensional $X Y$-model long ago (Kosterlitz, 1976) and used later in a number of works (Bulgadaev, 1984; Falci et al., 1995; Guinea and Schön, 1986). As the starting functional we take the tunneling action $S_{t}$

$$
S_{t}=\pi g \sum_{\langle i, j\rangle} \int_{0}^{\beta} \int_{0}^{\beta} d \tau d \tau^{\prime} \alpha\left(\tau-\tau^{\prime}\right) \sin ^{2}\left[\frac{\varphi_{i j}(\tau)-\varphi_{i j}\left(\tau^{\prime}\right)}{2}\right] .
$$

The charging part $S_{c}$ is not important for the renormalization group because it determines only the upper cutoff of integrations over frequencies. In the limit $T \rightarrow 0$ the function $\alpha\left(\tau-\tau^{\prime}\right)$ is proportional to $\left(\tau-\tau^{\prime}\right)^{-2}$ and the action is dimensionless. We neglect in this subsection the non-zero winding numbers $k_{i}$ and replace the phases $\varphi_{i}(\tau)$ by the variables $\phi_{i}(\tau)$, Eq. (2.47).

Following standard RG arguments we want to find how the form of the action $S_{t}$ changes under changing the cutoff. Generally speaking, it is not guaranteed that after integrating over the phases $\phi$ in an interval of the frequencies one comes to the same function $\sin ^{2} \phi$ in the action. The form of the functional may change, which would lead to a functional renormalization group.

In the present case appearance of terms $\sin ^{2} 2 \phi$, $\sin ^{2} 4 \phi$, etc. is not excluded and, indeed, they are generated in many loop approximations of the RG. Fortunately, the one loop approximation is simpler and the renormalization in this order results in a change of the effective coupling constant $g$ only.

To derive the RG equation we represent the phase $\phi$ in the form

$$
\phi_{i j \omega}=\phi_{i j \omega}^{(0)}+\bar{\phi}_{i j \omega}
$$


where the function $\phi_{i j \omega}^{(0)}$ is the slow variable and it is not equal to zero in an interval of the frequencies $0<$ $\omega<\lambda \omega_{c}$, while the function $\bar{\phi}_{i j \omega}$ is finite in the interval $\lambda \omega_{c}<\omega<\omega_{c}$, where $\lambda$ is in the interval $0<\lambda<1$. Substituting Eq. 2.66) into Eq. 2.65) we expand the action $S_{t}$ up to terms quadratic in $\bar{\phi}_{i j \omega}$. Integrating in the expression for the partition function

$$
Z=\int \exp \left(-S_{t}[\phi]\right) D \phi
$$

over the fast variable $\bar{\phi}_{i j \omega}$ with the logarithmic accuracy we come to a new renormalized effective action $\tilde{S}_{t}$

$$
\begin{aligned}
\tilde{S}_{t} & =2 \pi g \sum_{\langle i, j\rangle} \int_{0}^{\beta} \int_{0}^{\beta} d \tau d \tau^{\prime} \alpha\left(\tau-\tau^{\prime}\right) \\
& \times \sin ^{2}\left[\frac{\phi_{i j}(\tau)-\phi_{i j}\left(\tau^{\prime}\right)}{2}\right]\left(1-\frac{\xi}{2 \pi g d}\right)
\end{aligned}
$$

where $\xi=-\ln \lambda$. It follows from Eq. (2.67) that the form of the action is reproduced for any dimensionality $d$ of the lattice of the grains. This allows us to write immediately the following renormalization group equation

$$
\frac{\partial g(\xi)}{\partial \xi}=-\frac{1}{2 \pi d}
$$

The solution of Eq. 2.68 is simple. Neglecting the Coulomb interaction in the action $S_{t}$, Eq. (2.65), is justified only for energies smaller than $g E_{c}$ and this gives the upper cutoff. Then, the renormalized conductance $g(T)$ takes the form

$$
g(T)=g-\frac{1}{2 \pi d} \ln \frac{g E_{c}}{T} .
$$

and we come using Eq. (2.1) to Eq. (2.64) for the conductivity. Both the quantities depend on the temperature logarithmically.

Equation (2.69) is obtained in the one loop approximation and should be valid so long as the effective conductance $g(T)$ remains much larger than unity. This assumes that the result of the perturbation theory, Eq. 2.64 can be extended to a wider temperate interval and, in fact, it is valid as long as

$$
g-(2 \pi d)^{-1} \ln \left(g E_{c} / T\right) \gg 1 .
$$

At high temperatures, when the inequality (2.70) is fulfilled, one can speak of metallic behavior of the system. At lower temperatures $T<T_{c}$, where

$$
T_{c}=g E_{c} \exp (-2 \pi g d),
$$

the system is expected to show insulating properties.

The result for the conductivity correction (2.64) was obtained within a cubic lattice model. It is important to understand how it will change in a more realistic case of an irregular array. First, we note that Eq. (2.64) can easily be generalized to the case of an arbitrary periodic lattice with the result

$$
\sigma=\sigma_{0}\left(1-\frac{1}{\pi z g} \ln \left[\frac{g E_{c}}{T}\right]\right)
$$

where $z$ is the coordination number of the arbitrary periodic lattice.

The role of the dispersion of the tunneling conductance was studied in Feigel'man and Ioselevich (2005) within the $2 d$ model that assumed regular periodic positions and equal sizes of grains but random tunneling conductances. It was shown that the dependence (2.72) holds in a wide temperature range in the case of a moderately strong conductance dispersion. However, the distribution of the conductances broadens and this effect becomes especially important close to the metal-insulator transition where it was suggested to describe the transition in terms of percolation, (Feigel'man and Ioselevich, 2005). One may expect that in $3 d$ samples the effect of the conductance dispersion on the macroscopic transport will be less important than in $2 d$ ones, while, on the contrary, it will be more dramatic in $1 d$ samples.

Finally, we would like to comment on the validity of Eq. (2.72) in the case of an arbitrary irregular array. First, we note that in the RG approach the capacitance disorder leads to a local renormalization of the tunneling conductances. Then, the general irregular system can be viewed as an irregular array of equal size grains with random tunneling conductances. The main difference of such a system from that considered in (Feigel'man and Ioselevich, 2005) is that the coordination number of each grain varies from grain to grain. In such a case one expects that the coefficient in front of the second term in Eq. (2.72) will be determined by an effective coordination number in a system that is expected to be close to the average number of neighbors of a grain.

Following the RG scheme we did not take into account non-zero winding numbers $k_{i}$, Eqs. 2.44 - 2.47). This is a natural approximation because the contribution of such configurations should be exponentially small in $g$ ( $\sim \exp (-c g)$, where $c$ is of the order of unity). So long as the effective conductance $g$ is large in the process of the renormalization, the contribution of the non-zero winding numbers $k_{i}$ can be neglected. They become important when $g$ becomes of the order of unity. This is the region of the transition into the insulating state and apparently the non-zero winding numbers play the crucial role in forming this state. (However, the authors of recent publications (Altland et al., 2004, 2005; Mever et al., 2004) argued that the non-zero winding numbers might become important in $1 d$ samples at temperatures $T^{*}$ parametrically exceeding $T_{c}$, Eq. (2.71) ). 
(a)

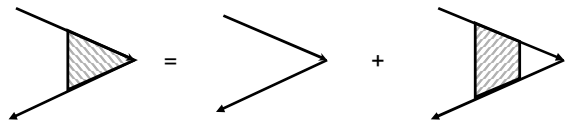

(b)

FIG. 10 Diagrams representing the vertex correction (a) and the renormalized Coulomb interaction (b).

\section{Metallic properties of granular arrays at low temperatures}

The phase functional technique may not be used for obtaining the conductivity corrections at temperatures $T \leq$ $\Gamma$ since in this regime the coherent electron motion over a large number of grains, which is missed in the functional approach (Ambegaokar et al., 1982), becomes important. In this section we will derive the conductivity correction within the diagrammatic perturbation theory described in the Sec. II.C.1 Unlike the phase functional approach, this technique does not allow us to obtain the non perturbative results in an easy way but it has an advantage of being applicable at arbitrary temperatures. In particular, we will see below that it reproduces at $T \gg \Gamma$ the perturbative result, Eq. 2.64). As we will show, the conductivity correction agrees in the low temperature regime with the one obtained in Refs. Altshuler and Aronov, 1985; Belitz and Kirkpatrick, 1994; Lee and Ramakrishnan, 1985) for homogeneously disordered samples

In Section II.C.1 we have already described the main building blocks of the diagrammatic technique including, in particular, the diffusion propagator defined by the ladder diagram in Fig. 8 The same ladder diagram describes the dressing of the interaction vertex as shown in Fig. 107. The dressed vertex can be used to obtain the polarization operator that defines the effective dynamically screened Coulomb interaction (Fig. 10p):

$$
V(\Omega, \mathbf{q})=\left[\frac{C(\mathbf{q})}{e^{2}}+\frac{2 \lambda_{\mathbf{q}}}{|\Omega|+\lambda_{\mathbf{q}} \Gamma}\right]^{-1}
$$

The conductivity of the granular metals is given by the analytical continuation of the Matsubara current-current correlation function. In the absence of the electronelectron interaction the conductivity is represented by the diagram (a) in Fig. 11 that results in high temperature (Drude) conductivity $\sigma_{0}$ defined in Eq. (2.1). First order interaction corrections to the conductivity are given by the diagrams (b-e) in Fig. 11] These diagrams are analogous to those considered in Altshuler and Aronov (1985) for the correction to the conductivity of homogeneous metals.

We consider the contributions from diagrams (b,c) and (d,e) separately: The sum of the diagrams (b,c) results

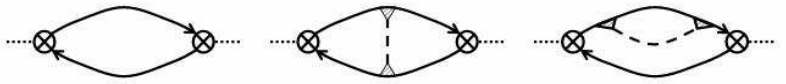

(a)

(b)

(c)

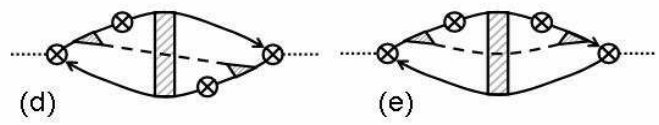

FIG. 11 Diagrams describing the conductivity of granular metals: the diagram (a) corresponds to $\sigma_{0}$ in Eq. 2.5 and it is the analog of Drude conductivity. Diagrams (b)-(e) describing first order correction to the conductivity of granular metals due to electron-electron interaction. The solid lines denote the propagator of electrons and the dashed lines describe effective screened electron-electron propagator. The sum of the diagrams (b) and (c) results in the conductivity correction $\delta \sigma_{1}$ in Eq. 2.5. The other two diagrams, (d) and (e) result in the correction $\delta \sigma_{2}$.

in the following correction to the conductivity

$$
\frac{\delta \sigma_{1}}{\sigma_{0}}=-\frac{1}{2 \pi d g} \operatorname{Im} \sum_{\mathbf{q}} \int d \omega \gamma(\omega) \lambda_{\mathbf{q}} \tilde{V}(\omega, \mathbf{q}),
$$

where $\gamma(\omega)=\frac{d}{d \omega} \omega \operatorname{coth} \frac{\omega}{2 T}$, and the potential $\tilde{V}(\omega, \mathbf{q})$ is the analytic continuation of the screened Coulomb potential $V(\Omega, \mathbf{q})$ with dressed interaction vertices including those attached at the both ends

$$
\tilde{V}(\omega, \mathbf{q})=\frac{2 E_{c}(\mathbf{q})}{\left(\lambda_{\mathbf{q}} \Gamma-i \omega\right)\left(4 \lambda_{\mathbf{q}} E_{c}(\mathbf{q})-i \omega\right)} .
$$

The expression for the screened Coulomb interaction $\tilde{V}(\omega, \mathbf{q})$, Eq. (2.75), is written in a simplified form using the fact that the charging energy $E_{c}(\mathbf{q})=e^{2} / 2 C(\mathbf{q})$, expressed in terms of the Fourier transform of the capacitance matrix $C(\mathbf{q})$, is much larger than the escape rate $\Gamma$.

Performing the integration over the frequency and summing over the quasimomentum $\mathbf{q}$ in Eq. (2.74) with the logarithmic accuracy we obtain the correction to the conductivity, Eq. (2.6). One can see from Eq. (2.74) that the contribution $\delta \sigma_{1}$ in Eq. (2.6) comes from the large energy scales, $\varepsilon \geq \Gamma$. At low temperatures $T \leq \Gamma$, the logarithm is cut off by the energy $\Gamma$ and is no longer temperature dependent.

To obtain the total correction to the conductivity of a granular sample the two other diagrams, (d) and (e) in Fig. 11 should be taken into account. These diagrams result in the following contribution to the conductivity (Beloborodov et al., 2003)

$$
\frac{\delta \sigma_{2}}{\sigma_{0}}=-\frac{2 g \delta}{\pi d} \sum_{\mathbf{q}} \int d \omega \gamma(\omega) \operatorname{Im} \frac{\tilde{V}(\omega, \mathbf{q}) \sum_{\mathbf{a}} \sin ^{2}(\mathbf{q a})}{\lambda_{\mathbf{q}} \Gamma-i \omega}
$$

In contrast to the contribution $\delta \sigma_{1}$, Eq. (2.74), the main contribution to the sum over the quasimomentum $\mathbf{q}$ in Eq. 2.76 comes from the low momenta, $q \ll 1 / a$. In this regime the capacitance matrix $C(\mathbf{q})$ in Eqs. (2.75) 
and (2.76) has the asymptotic form

$$
C^{-1}(\mathbf{q})=\frac{2}{a^{d}} \begin{cases}\ln (1 / q a) & d=1, \\ \pi / q & d=2, \\ 2 \pi / q^{2} & d=3\end{cases}
$$

Using Eqs. (2.75- 2.77), we obtain the result for the correction $\delta \sigma_{2}$ in Eq. (2.7). This correction has a physical meaning similar to that of the Altshuler-Aronov correction (Altshuler and Aronov, 1985) derived for homogeneously disordered metals.

Comparing results in Eqs. (2.5) - 2.7) with those obtained in the previous subsection using the AES functional one can see that the correction to the conductivity obtained in Sec. II.C is equivalent to the correction $\delta \sigma_{1}$ in Eq. (2.5), which corresponds in the diagrammatic approach to the sum of diagrams (b) and (c) in Fig. 11] The correction $\delta \sigma_{2}$ in Eq. (2.5) becomes important only at low temperatures, $T \leq \Gamma$ where AES functional is not applicable.

Using the final results of the calculations, Eqs. (2.6) and (2.7), we can make a very important statement about the existence of a metal-insulator transition in three dimensions. It follows from Eq. (2.7) that for a $3 d$ granular array there are no essential corrections to the conductivity at low temperatures $T \ll \Gamma$ coming from the low energies $\omega \leq \Gamma$, since the correction $\delta \sigma_{2}$ is always small. This means that the result for the renormalized conductance, Eq. (2.69), for $3 d$ samples can be written within the logarithmic accuracy in the form

$$
\tilde{g}(T)=g-\frac{1}{6 \pi} \ln \left[\frac{g E_{C}}{\max (\tilde{g} \delta, T)}\right],
$$

such that it is valid for all temperatures as long as the renormalized conductance is large, $\tilde{g}(T) \gg 1$.

One can see from Eq. (2.78) that for a large bare conductance, $g \gg(1 / 6 \pi) \ln \left(g E_{C} / \delta\right)$, the renormalized conductance $\tilde{g}$ is always large and the system remains metallic down to zero temperatures. In the opposite limit $g<(1 / 6 \pi) \ln \left(g E_{C} / \delta\right)$, the system flows when decreasing the temperature to the strong coupling regime, $\tilde{g} \sim 1$, which indicates the onset of the insulating phase. One can see that with the logarithmic accuracy the critical value of the conductance $g_{c}$ is given by Eq. (2.12), and this value separates the metallic and insulating states (Beloborodov et al., 2003).

One can see from Eq. (2.7) that in $1 d$ and $2 d$ samples the correction to the conductivity, being negative, grows with decreasing the temperature and diverges in the limit $T \rightarrow 0$. Such a behavior is usually attributed to a localization in the limit $T \rightarrow 0$.

However, the situation is more interesting. In a recent paper (Basko et al., 2005), it was demonstrated that there must be a metal-insulator transition at finite temperature $T_{k}$ in systems with a weak repulsion provided all one-particle states are localized. This means that the conductivity is strictly zero at $T<T_{k}$ and becomes finite at $T>T_{k}$. The $1 d$ and $2 d$ granular systems with the Coulomb interaction discussed in the present review should belong to the class of the models considered in (Basko et al., 2005) (all one-particle states should be localized in $1 d$ and $2 d$ systems for any disorder) and one can expect such a transition. Clearly, the results of Eq. (2.7) should hold for $T \gg T_{k}$.

At the same time, the $3 d$ samples do not fall into the class of the models studied by Basko et al. (2005) because there exist both localized and extended one-particle states. Therefore, for $3 d$ granular systems, one can speak of the metal-insulator transition at $T=0$ only. The transition point can be varied by changing, e.g. the tunneling conductance between the grains or their size.

Finally, we would like to note that the low temperature conductivity correction $\delta \sigma_{2}$ is less sensitive to a particular model of a granular sample than the high temperature result, Eq. (2.64). As we will discus in the next section, the low temperature conductivity correction is determined by the scales larger than the single grain size and can be expressed in terms of the effective diffusion coefficient $D_{\text {eff }}$. This assumes that the underlying structure of the model is not important in this regime.

\section{E. Universal description of granular materials}

One can see from the previous subsection that at low temperatures, $T \ll \Gamma$, the dependence of the correction $\delta \sigma_{2}$, Eq. 2.7), to the conductivity of granular metals coincides exactly with the corresponding result for the conductivity of the homogeneously disordered samples. A question immediately arises: is it an accidental coincidence that the two different physical systems exhibit in the main approximation the identical low temperature transport behavior, or there is an underlying deep connection between the two? Can on describe both the region $T \leq \Gamma$ and $T \geq \Gamma$ in an unified manner? The main goal of this subsection is to answer these important questions.

A very convenient description of the low temperature behavior of homogeneously disordered metals is based on the so-called $\sigma$-model. There are several formulations of the $\sigma$-model based on the replica trick (Efetov et al., 1980; Wegner, 1979), supersymmetry (Efetov, 1983, 1997), and Keldysh Green functions (Kamenev and Andreev, 1999). The approach of Efetov et al. (1980) has been generalized by Finkelstein (1990) to include the interaction effects. It is clear that the $\sigma$-model of Finkelstein (1990) cannot be used for the granular materials because it does not contain the scale $\Gamma$.

Fortunately, the approach of Finkelstein (1990) can rather easily extended to include the granularity. This can be done using Eqs. (2.15) - (2.23) and following the usual way (Andreev and Beloborodov, 2004; Beloborodov et al., 2001): 1) we write a generating functional in terms of functional integrals over anticommuting Grassmann variables, 2) average over the disorder 
using a replica trick, 3) we decouple a $\psi^{4}$ effective interaction appearing after the averaging by a Gaussian integration over a $Q$ - matrix field and do the same for the Coulomb interaction term in Eq. (2.23) using axillary fields $\bar{V}$ (Efetov et al., 1980; Finkelstein, 1990), 4) we integrate thus obtained exponential of a quadratic form over the $\psi$ fields and finally, 5) find a saddle point in the free energy functional containing the fields $Q$ and $\bar{V}$ only and expand around this saddle point. The final expression for the effective low-energy action reads:

$$
\begin{aligned}
S[Q, V]= & -\frac{\pi}{2 \delta} \sum_{i} \operatorname{Tr}\left[\left(\hat{\varepsilon}+\bar{V}_{i}\right) Q_{i}\right] \\
& -\frac{\pi g}{8} \sum_{\langle i, j\rangle} \operatorname{Tr}\left[Q_{i} Q_{j}\right]+\frac{1}{2 e^{2}} \sum_{i, j} C_{i j}\left(\bar{V}_{i} \bar{V}_{j}\right) .
\end{aligned}
$$

Here the sums are performed over the grain indices, the symbol $\langle\ldots\rangle$ means summation over the nearest neighbors, $C_{i j}$ is the capacitance matrix, $\hat{\varepsilon}=i \partial_{\tau}$ and the symbol "Tr" means the trace over spin and replica indices and integration over $\tau$. The field $\bar{V}$ in Eq. (2.79) is a time dependent vector in the replica space and the corresponding "scalar product" is implied: $\left(\bar{V}_{i} \bar{V}_{j}\right)=$ $\sum_{\alpha} \int_{0}^{1 / T} \bar{V}_{i \alpha}(\tau) \bar{V}_{j \alpha}(\tau) d \tau$, where $\alpha$ is the replica in$\operatorname{dex}(0 \leq \alpha \leq N)$. The $Q$ - matrix in Eq. (2.79) is the matrix in the time (it depends on two times $\tau$ and $\tau^{\prime}$ ), spin, and replica spaces subject to the constraints $Q^{2}=1, \operatorname{Tr} Q=0$. The variable $\tau$ enters $Q$ in the same way as the replica and spin ones, which means that $\left(Q_{1} Q_{2}\right)_{\tau, \tau^{\prime}} \equiv \int Q_{1}\left(\tau, \tau^{\prime \prime}\right) Q_{2}\left(\tau^{\prime \prime}, \tau^{\prime}\right) d \tau^{\prime \prime}$.

For energies smaller than the Thouless energy $E_{T h}$ of one grain, Eq. (1.5), the $Q$-matrices in Eq. (2.79) are coordinate independent within each grain. They can be written in the form

$$
Q_{i}=U_{i} \Lambda U_{i}^{-1}
$$

where the function $\Lambda$ equals

$$
\Lambda_{\tau, \tau^{\prime}}=\frac{i T}{\sin \pi T\left(\tau-\tau^{\prime}\right)},
$$

and $U_{i}$ is an unitary matrix. The action $S[Q, \bar{V}]$ in Eq. 2.79) describes the entire region of both low, $T \leq \Gamma$, and not very low, $T \geq \Gamma$, temperatures discussed in the previous subsections. For $T \gg \Gamma$, an essential contribution comes from $U\left(\tau, \tau^{\prime}\right)$ in Eq. (2.80) diagonal in time, spin and replica spaces. This means that we write this function as

$$
U_{i}\left(\tau, \tau^{\prime}\right)=\delta_{\alpha \beta} \delta\left(\tau-\tau^{\prime}\right) \exp \left[i \varphi_{\alpha i}(\tau)\right]
$$

where $\alpha, \beta$ stand for both replica and spin indices.

Substituting Eqs. (2.80 2.82) into Eq. (2.79), calculating the integral

$$
\int \exp (-S[Q, \bar{V}]) D \bar{V}
$$

and taking the limit $N=0$, which is trivial in this limit, we come to the AES action, Eqs. 2.55 2.58).

The $\sigma$-model, Eq. (2.79), has a well-defined continuum limit. It can be obtained for slow spatial variations of the $Q$-matrix by expanding the second term in Eq. (2.79) to the second order in gradients of $Q$, and replacing the summation over $i$ by the integration over $\mathbf{r}$. The tunneling term in Eq. (2.79) describes in the continuum limit the diffusion and we reduce the action, Eq. (2.79), to the form

$$
\begin{aligned}
S & =-\pi \nu \int \operatorname{Tr}\left[(\hat{\varepsilon}+\bar{V}) Q-\frac{D}{4}(\nabla Q)^{2}\right] d \mathbf{r} \\
& +\int \frac{d \mathbf{r} d \mathbf{r}^{\prime}}{a^{2 d}} \operatorname{Tr}\left[\bar{V}_{\mathbf{r}} \frac{C_{\mathbf{r r}}}{2 e^{2}} \bar{V}_{\mathbf{r}^{\prime}}\right] .
\end{aligned}
$$

where $\nu$ is the density of states. The coefficient $D$ in the second term of Eq. (2.83) is in this approximation just the classical diffusion coefficient determined by Eq. (2.3).

However, we can derive Eq. (2.83) more accurately, not just neglecting the contribution of energies exceeding $\Gamma$ but taking them into account. Calculating the contribution of the energies exceeding $\Gamma$ we can use Eq. (2.82) for the excitations with such energies. As a result, we come again to Eq. (2.83) but with a renormalized coefficient $D$ that can be written in the form

$$
D=g_{\text {eff }} a^{2} \delta
$$

where $g_{\text {eff }}$ equals (c.f. with Eq. (2.69)

$$
g_{e f f}=g-\frac{1}{2 \pi d} \ln \frac{E_{c}}{\delta} .
$$

Since the effective model (2.83) operates with matrix fields $Q$ that have only long range degrees of freedom, it applies after an appropriate high energy renormalization to any disordered metal including a homogeneously disordered one. Integrating over the potentials $\bar{V}$ one can come to the $\sigma$-model of Finkelstein (1990) (the limit of a long range interaction is implied).

Thus, all the information about the granularity of the sample is hidden in the low temperature limit in the coefficients of the effective model (2.83). The conductivity of the sample is related to the effective diffusion coefficient through the usual Einstein relation

$$
\sigma=2 e^{2} D\left(a^{d} \delta\right)^{-1} .
$$

The effective model, Eq. (2.83), together with equation (2.69) for the renormalized conductance naturally explains the results for the low temperature, $T \ll \Gamma$, conductivity described in section II.D The interaction correction to conductivity consists of two terms: 1) The first contribution is temperature independent and comes from the high energy renormalization of the diffusion coefficient D, Eqs. (2.86 2.87). It can be written in the form (c.f. with Eq. (2.6) )

$$
\delta \sigma_{1}=-\sigma_{0} \frac{1}{2 \pi d g} \ln \left[\frac{E_{c}}{\delta}\right],
$$


where $\sigma_{0}$ is the classical Drude conductivity defined in Eq. (2.1). This contribution is specific for the granular materials.

2) The second contribution to conductivity, Eq. (2.7), is temperature dependent and comes from the low energy renormalization of the diffusion coefficient $D$ in the effective model (2.83). It coincides with the corresponding correction to conductivity obtained for homogeneously disordered metals (Altshuler and Aronov, 1985).

Making use of the effective description of the granular metals in terms of effective the $\sigma$-model, Eq. (2.83), applicable at low temperatures we conclude: all the phenomena that are described in terms this model including localization effects, magnetoresistance, Hall conductivity, etc., are universal.

Using the $\sigma$-model, Eq. (2.83), we also describe quantum interference (weak localization) corrections to the conductivity, (Gorkov et al., 1979). In the leading order in the inverse tunneling conductance, $1 / \mathrm{g}$, the interaction and weak localization corrections can be considered independently. The quantum interference corrections may be obtained from the effective $\sigma$-model, Eq. (2.83), using directly the corresponding results for homogeneously disordered metals (Efetov et al., 1980) with the proper effective diffusion coefficient, $D=g a^{2} \delta$. For $2 D$ and $1 D$ samples it is important to take into account dephasing effects since the weak localization correction diverges. The dephasing time $\tau_{\phi}$ can also be extracted directly from the results for homogeneously disordered metals (Altshuler et al., 1982) with the proper effective diffusion coefficient, $D$. The final result for the weak localization corrections is given by Eqs. (2.8) and (2.9).

\section{F. Insulating Properties of Granular Metals: Periodic model}

In the previous section we discussed the transport properties of metallic granular arrays with strong intergranular tunneling coupling. Now we turn to the opposite limit of weakly coupled grains, $g \ll 1$. We start our consideration with a detailed description of a periodic granular array model that is presented in this section. To remind, the periodic model assumes periodic arrangements of equal size grains as well as the absence of the disorder in conductances and grain potentials. At the same time, the electron motion inside the grain is chaotic and this brings a disorder into the model.

As we discussed in the Introduction, the periodic granular array model predicts the insulating conductivity behavior with a hard gap in the electron excitation spectrum and, for this reason, it cannot describe the transport properties of realistic granular systems where the conductivity governed by the variable range hopping mechanism. Nevertheless, it is instructive to consider this model for two reasons: first, it is important to be see explicitly that such a model may indeed produce only the activation conductivity dependence; second, this model illustrates a general effect of the charge renormalization of the Coulomb energy due to the intergranular coupling.

\section{Activation conductivity behavior}

First, we will consider a periodic granular array with a small intergranular coupling, $g \ll 1$. The conductivity in this case can be found via the gauge transformation technique described in Sec. II.B.3. The phase action in the lowest order in tunneling conductance $g$ is simply given by the Coulomb term $S_{c}$, Eq. (2.56). With the help of the Kubo formula the conductivity in the first nonvanishing order in $g$ may be written as (Efetov and Tschersich, 2003)

$$
\sigma(\omega)=\frac{2 \pi e^{2} g T^{2} i a^{2-d}}{\omega} \int_{0}^{\beta} d \tau \frac{1-\exp i \Omega \tau}{\sin ^{2}(\pi T \tau)} \Pi(\tau)
$$

Here the analytic continuation to the real frequency is assumed as $\Omega \rightarrow-i \omega$ and the function $\Pi(\tau)$ represents the phase correlation function

$$
\Pi\left(\tau_{1}-\tau_{2}\right)=\left\langle\exp \left(i\left(\varphi\left(\tau_{1}\right)-\varphi\left(\tau_{2}\right)\right)\right)\right\rangle
$$

where the averaging is performed with the Coulomb action $S_{c}$ in Eq. (2.56).

At first glance, calculation of the correlation function $\Pi\left(\tau_{1}-\tau_{2}\right)$ with the action $S_{c}$ reduces to computation of a Gaussian integral. However, it is not so because at finite temperatures it is necessary to take into account all winding numbers, Eq. (2.47), in order to obtain the correlation function $\Pi(\tau)$ correctly. At low temperature the straightforward calculation results in (Efetov and Tschersich, 2003)

$$
\sigma=2 \sigma_{0} \exp \left(-E_{c} / T\right)
$$

where $\sigma_{0}$ is the Drude high temperature conductivity and $E_{c}$ is the single grain charging energy. This result has a clear physical meaning: The conductivity of the insulating granular array is mediated by electrons and holes that are present in the system as real excitations. Their concentration is given by the Gibbs law and the factor 2 appears due to the presence of both electrons and holes. From the result (2.90) we conclude that the activation exponent is determined by the Mott gap - that is the energy one pays to add or remove an electron from the system.

One can extend the calculation of the conductivity to higher orders in $g$ expanding in the tunneling term $S_{t}$, Eq. (2.57). It was shown by Loh et al. (2005) that the activation behavior persists at least in the next order in $g$. Apparently, the activation behavior, Eq. (2.13) is quite universal for the periodic arrays and what one should clarify is the dependence of the Mott gap $\Delta_{M}$ on the conductance $g$. Now we turn to this question.

\section{Mott gap at small tunneling conductances}

At finite intergranular coupling the Mott gap turns out to be reduced due to the processes of virtual tunneling 
of electrons to the neighboring grains. This effect can be most easily studied in the limit of the low coupling between the grains within the straightforward perturbation theory in the tunneling conductance. The gap $\Delta_{M}$ can be formally defined as

$$
\Delta_{M}=E_{N=1}-\mu-E_{N=0},
$$

where $\mu$ is the chemical potential, $E_{N=0}$ is the ground state energy of the charge neutral array and $E_{N=1}$ is the minimal energy of the system with an extra electron added to the neutral state. The correction to the Mott gap due the to the electron tunneling to the neighboring grains can be found in the second order of the perturbation theory

$$
\Delta E=-\sum_{k} \frac{\left|V_{k, 0}\right|^{2}}{E_{k}-E_{0}}
$$

where the matrix elements of a perturbation $V$ are taken between the ground state 0 and excited states $k$.

The correction to the energy due to the finite intergranular coupling should be included in both the terms $E_{N=1}$ and $E_{N=0}$ in Eq. (2.91). We consider in the zero approximation isolated grains and the tunneling Hamiltonian $\hat{H}_{t}$, Eq. (2.24), is our small perturbation. Using Eq. (2.92) we have to calculate matrix elements of the tunneling between neigboring grains. As all grains are equivalent, we consider the tunneling between the grains 1 and 2 assuming that the charge $N$ on the grain 1 can be 0 or 1 , while the grain 2 is initially neutral. Contribution of the hops between the grain 1 and all its other neighbors leads merely to the factor $z$ (coordination number) in the final result. Using Eq. (2.92) we should calculate the corrections to the energies $E_{N=0}$ and $E_{N=1}$.

First, let us consider the correction to the ground state energy $E_{N=0}$. In this case the matrix elements $V_{k, 0}$ correspond to electron tunneling between the initially neutral neighboring grains. The excitation energy of this process equals $\varepsilon_{k_{1}}+\varepsilon_{k_{2}}+E_{e h}$, where $\varepsilon_{k_{2}}$ is the bare (with no Coulomb energy included) energy of an electron excitation in grain $2, \varepsilon_{k_{1}}$ is the bare energy of a hole excitation in grain 1 , and $E_{e h}$ is the electrostatic energy the electron-hole excitation that should be determined from Eq. (2.23). It is equal to $E_{e h}=E_{11}^{c}+E_{22}^{c}-2 E_{12}^{c}$, where $E_{i j}$ can be obtained from Eq. (2.23) putting $n_{i}=1$ and $n_{j}=-1$. For the periodic array of the grains under consideration, the energy $E_{e h}$ reduces to

$$
E_{e h}=2 E_{c}-2 E_{12}^{c}
$$

The energy correction corresponding to such a process is

$$
-\Delta E_{N=0}=2 \sum_{k_{1}, k_{2}} \frac{\left|t_{k_{1} k_{2}}\right|^{2}}{\varepsilon_{k_{1}}+\varepsilon_{k_{2}}+E_{e h}}
$$

where the factor 2 takes into account the equivalent process of the electron hopping from grain 2 to grain 1 .
Analogously, we find the correction to the energy $E_{N=1}$. The excitation energy of the process of the electron tunneling from the grain 2 to the grain 1 equals $\varepsilon_{k 1}+\varepsilon_{k_{2}}+3 E_{11}^{c}+E_{22}^{c}-4 E_{12}^{c}=\varepsilon_{k_{1}}+\varepsilon_{k_{2}}+2 E_{e h}$, while the excitation energy of the process of electron tunneling from the grain 1 to the grain 2 is $\varepsilon_{k_{1}}+\varepsilon_{k_{2}}+E_{11}^{c}-E_{22}^{c}=$ $\varepsilon_{k_{1}}+\varepsilon_{k_{2}}$. The corresponding correction to the energy $E_{N=1}$ reads

$$
-\Delta E_{N=1}=\sum_{k_{1}, k_{2}} \frac{\left|t_{k_{1} k_{2}}\right|^{2}}{\varepsilon_{k_{1}}+\varepsilon_{k_{2}}+2 E_{e h}}+\frac{\left|t_{k_{1} k_{2}}\right|^{2}}{\varepsilon_{k_{1}}+\varepsilon_{k_{2}}} .
$$

One can see that corrections to the energy levels, Eqs. 2.94 2.95), are ultraviolet divergent. However, their difference is finite and it gives the correction to the Mott gap, $\Delta_{M}$. Subtracting Eq. (2.94) from Eq. (2.95), replacing the summation over the states by integrals over a continuous variable $\varepsilon$, such that $\varepsilon_{k_{1}\left(k_{2}\right)} \rightarrow \varepsilon_{1(2)}$, we can easily caclulate the Mott gap, $\Delta_{M}$. Taking into account the spin degeneracy we obtain the following expression for the Mott gap (Beloborodov et al., 2005c)

$$
\Delta_{M}=E_{c}-\frac{2 z}{\pi} g E_{e h} \ln 2,
$$

where $z$ is the coordination number of the array of the grains and $E_{e h}$ is the energy necessary to create an electron-hole excitation in the system by removing an electron from a given grain and putting it on a neighboring one. In the case of a diagonal Coulomb interaction $E_{i j}=E_{c} \delta_{i j}$ the energy of an electron-hole excitation $E_{e h}$ is simply twice the Coulomb charging energy $E_{c}$.

Derivation of Eq. (2.96) was carried out neglecting the fact that an extra electron added to the neutral system in the presence of a finite intergranular coupling can move diffusively over the sample. Contributions corresponding to such processes are suppressed by an extra small factor $\delta / E_{c} \ll 1$ and, thus, can be neglected. More details of the calculations can be found in Ref. (Beloborodov et al., 2005c).

\section{Mott gap at large tunneling conductances}

From Eq. (2.96) one can see that the Mott gap $\Delta_{M}$ is significantly reduced at values $g z \sim 1$, where the perturbation theory becomes inapplicable. Suppression of the gap $\Delta_{M}$ at large tunneling conductances $g$ can be found with the help of the phase action, Eqs. (2.55 - 2.57). In fact, the renormalization of the charging energy at $g z \gg 1$ can be obtained with the renormalization group approach that we have described in subsection II.C.2

Indeed, the RG Eq. (2.68) is written assuming that $g$ is a function of the independent variable $\xi$. We can invert this equation and assume that $\xi$ is a function of $g$. The running variable $\xi$ determines an effective charging energy $E_{c}^{e f f}$ as $\xi=\ln E_{c}^{e f f}$ and we obtain solving the latter equation

$$
E_{c}^{e f f} \sim A \exp (-\pi g z)
$$


where $A$ is a constant.

In order to determine the Mott gap $\Delta_{M}$ we notice that at conductances $g z \sim 1$ the charging energy is of the order of the Mott gap $\Delta_{M}$, as one can see from Eq. (2.96). Matching Eq. (2.97) with Eq. (2.96) we conclude that the Mott gap $\Delta_{M}$ at large $g z$ is reduced exponentially and can be written as

$$
\Delta_{M} \sim E_{c} \exp (-\pi g z)
$$

The RG approach used to obtain Eq. (2.98) is applicable only at energy scales larger than the inverse escape rate from a single grains $\Gamma$, which means that Eq. (2.98) is valid as long as the Mott gap $\Delta_{M}$ is larger than $\Gamma$. In this region the system should be the insulator. In the opposite limit one can expect that the granular material becomes a metal.

Taking $\Delta_{M} \sim \Gamma$ and resolving Eq. (2.98) with respect to the conductance $g$ we obtain the critical conductance $g_{c}$, Eq. (2.12), that marks the boundary between the insulating and metallic phases at low temperature. Thus, we come to the conclusion that the Mott gap $\Delta_{M}$ remains finite as long as $g<g_{c}$, which assumes the activation behavior, Eq. (2.13). The Arrhenius law, Eq. (2.13), is typical for crystalline insulators.

\section{Metal - Insulator transition in periodic granular arrays}

In the previous subsection we have shown that in the case of three dimensional arrays there is a critical conductance $g_{c}$, such that the samples with $g<g_{c}$ are insulators at $T \rightarrow 0$, while those with $g>g_{c}$ are metals. The analysis was performed from both metallic and insulator sides and both the approaches agree as concerns the value of the critical conductance $g_{c}$, Eq. (2.12). Unfortunately, they are not applicable in the vicinity of the phase transition and do not allow us to find the critical behavior of the Mott gap $\Delta_{M}$ near $g_{c}$.

The order of the Mott transition in $3 d$ periodic granular array is also not known, since one cannot exclude a weakly first order phase transition. For, example the Mott transition in the Hubbard model in dimensionality $d \geq 3$ is believed to be of the first order, and since physics of the transition is similar, the same can be expected for the periodic granular array. We note however that in spite of the similarities between the periodic granular array model and the Hubbard model, there are essential differences between the two. Namely, in granular metals, even in the case of the periodic samples, the electron motion within a grain as well as on the scales exceeding the intergranular distance is diffusive, while in the Hubbard model electrons move through a periodic lattice that allows to label their states by quasimomenta.

The model of the granular metals has also an additional physical parameter $\delta$ - the mean energy level spacing in a single grain, that has no analog in the Hubbard model. Yet, the physics of the transition looks similar in both the cases, and one may expect that the methods developed for the study of the Mott transition in the system of strongly interacting electrons on the lattice.

In the case of $1 d$ and $2 d$ granular samples the question of the Mott transition at $T \rightarrow 0$ cannot be formulated since, even in the metallic phase, the conductivity corrections are divergent at low temperatures, see Eqs. (2.6)-(2.9). However, the critical conductance $g_{c}$ still has a meaning of the boundary between the hard gap insulator behavior at $g<g_{c}$ and a weak insulating behavior $g>g_{c}$ that essentially coincide with insulating properties of the ordinary disordered metals. We note that the fate of the ordinary disordered metals with interaction at low temperature is not quite clear due to difficulties related to the divergence of the conductivity corrections (Finkelstein, 1990). In particular, the metal to insulator transition recently observed in disordered films (Abrahams et al., 2001; Kravchenko and Sarachik, 2004) has not been yet theoretically understood, although some plausible scenarios have been suggested recently (see, e.g. (Punnoose and Finkelstein, 2005)).

It is clear that understanding the metal-insulator transition in the granular materials is one of the most difficult theoretical problems and considerable efforts are necessary to solve it.

\section{G. Hopping conductivity in Granular Materials}

In the previous section we have shown that a strictly periodic granular model predicts and explains the activation conductivity behavior only. Experimentally observed temperature behavior of the conductivity of granular metals and arrays of quantum dots at low temperatures, however, is usually not of the activation type but resembles the Efros-Shklovskii law, Eq. (1.1), (Liao et al., 2005; Romero and Drndic, 2005; Tran et al., 2005; Yakimov et al., 2003; Yu et al., 2004). As we have already discussed in the introduction, it is crucial for understanding the hopping transport in granular metals to take into account an electrostatic disorder that seems to be present in any realistic system. This disorder can be viewed as a random potential $V_{i}$ applied to each grain that lifts the Coulomb blockade on a part of grains in the sample such that the conductivity is mediated by the electron hopping between the sites where the Coulomb blockade is almost removed. Below, we consider in more details the two essential ingredients of the hopping conductivity - the density of states and the mechanisms of the electron tunneling through the dense granular system in different regimes.

\section{Density of states}

The electrostatic disorder causes fluctuations in the electrostatic energy of granules and can thus lift the Coulomb blockade at some sites of the granular sam- 
ple. This results, in its turn, in the finite density of states at the Fermi level and makes the variable range hopping the dominating mechanism of conductivity. The bare density of states induced by the random potential can be substantially suppressed due to the presence of the long-rang Coulomb interaction in the same way as it happens in semiconductors where the density of states (DOS) $\nu_{a}(\varepsilon)$ is given by the Efros-Shklovskii expression (Efros and Shklovskii, 1975; Shklovskii and Efros, 1988)

$$
\nu_{g}(\varepsilon) \sim\left(\tilde{\kappa} / e^{2}\right)^{d}|\varepsilon|^{d-1},
$$

with $e$ being the electron charge and $\tilde{\kappa}$ being the dielectric constant. This result has recently been confirmed by Muller and Ioffe (2004); Pankov and Dobrosavlijevic (2005) analytically using a locator approximation.

As we have explained in Sec. II.B the Coulomb interaction in the granular matter may be considered writing classical electrostatic formulae for the electron-electron interaction, Eq. (2.23). This approach can also be used in the limit of a weak coupling between the grains, $g \ll 1$. So, we describe the Coulomb interaction in the presence of the electrostatic disorder writing the following expression for the Hamiltonian $H$

$$
H=\sum_{i} V_{i} n_{i}+\sum_{i j} n_{i} E_{i j}^{c} n_{j},
$$

where $V_{i}$ is the random external potential, $n_{i}$ is the number of excess electrons on the grain $i$, and $E_{i j}^{c}$ is the Coulomb interaction between the grains $i$ and $j$. Taking into account the asymptotic behavior of the Coulomb interaction matrix $E_{i j}^{c} \sim e^{2} / 2 r_{i j} \tilde{\kappa}$, where $r_{i j}$ is the distance between the grains $i$ and $j$ and $\tilde{\kappa}$ is the effective dielectric constant of the granular sample we see that the classical model (2.100) for the granular materials is essentially equivalent to the one studied by Efros and Shklovskii. Therefore their results have to be applicable to the model of the granular array, Eq. (2.100), as well.

At the same time, one expects that the DOS in the array of the metallic granules is larger than that in a semiconductor since each metallic grain has a dense electron spectrum. Indeed, one has to remember that there are many electron states in a grain that correspond to the same grain charge, while in the model of impurity levels in the semiconductors the charge is uniquely (up to the spin) identified with the electron state.

The energy of an unoccupied state $\varepsilon_{i}$ in the model specified by Eq. 2.100) is by definition the energy of an electron placed on this state. In the granular metal, any state with the energy larger than $\varepsilon_{i}$ is also available for the electron. Thus, in order to translate the ES result, Eq. (2.99), to the density of electron spectrum in granular metals one has to integrate the dependence (2.99) over the energy $\varepsilon$ and multiply it by the bare DOS in a single grain. As a result, we obtain

$$
\nu(\varepsilon) \sim \nu_{0}\left(|\varepsilon| \tilde{\kappa} / e^{2}\right)^{d}
$$

where $\nu_{0}$ is the average DOS in a single grain (defined as the number of states per energy). However, the above expression cannot be used in the Mott argument for the hopping conductivity where one needs to estimate the distance to the first available state $r$ within the energy shell $\varepsilon$ via the relation

$$
r^{d} \int_{0}^{\varepsilon} d \varepsilon^{\prime} \nu_{g}\left(\varepsilon^{\prime}\right) \sim 1
$$

The problem with using the expression for DOS, Eq. (2.101), in Eq. (2.102) is that the expression (2.101) takes into account the fact that if there is a state available for placement of an electron with a given energy then typically on the same grain, there will be plenty of other states available for electron placement. However, for application to the hopping conductivity we should not count different electron states that belong to the same grain since it is enough to find at least one state to insure the transport.

Thus, when finding DOS relevant for the hopping transport, the lowest energy states within each grain are to be counted only. Then, we arrive at the conclusion that, although the electron DOS in granular metals is modified according to Eq. (2.101), one has to use the ES expression for DOS in its form (2.99) even in granular metals in order to find the distance to the first available state within a given energy shell via Eq. (2.102).

Similar considerations were presented in Zhang and Shklovskii (2004). Following this reference we call the DOS that counts only the lowest excited states in each grain and that is relevant for the hopping conductivity "the density of ground states". In order to distinguish this quantity from the conventional density of states from Eq. (2.101) we ascribe to it the subscript $g$ as in Eq. (2.99).

The presented arguments demonstrate that one can obtain a finite density of states in a granular material in the insulating regime. Although this is the necessary ingredient for establishing the mechanism of the hopping conductivity, it alone is not sufficient for this type of transport. The problem is that en electron has to hop over several grains, which, at first glance, does not look probable in the case of closely packed granular array. For quite a long time, this fact did not allow to apply the Efros-Shklovskii theory for explanation of the behavior, Eq. (1.1), in the granular materials and a rather artificial model of Abeles et al. (1975) was used.

Only recently a resolution of this puzzle has been suggested independently in publications (Beloborodov et al., 2005c; Feigel'man and Ioselevich, 2005). The authors of these works demonstrated that a well known phenomenon of co-tunneling (virtual hops) (Averin and Nazarov, 1992, 1990; Averin and Likharev, 1991) through a grain might be responsible for the long range hops of the electrons. One has to distinguish between elastic and inelastic co-tunneling processes. The elastic co-tunneling is the dominant mechanism for the hopping conductivity at low 
temperatures $T<T_{\text {cross }}$, while at larger temperatures $T>T_{\text {cross }}$ the electron transport goes via inelastic co-tunneling processes. The characteristic temperature $T_{\text {cross }}$ of the crossover from the elastic to the inelastic tunneling is given by the following formula

$$
T_{\text {cross }}=\bar{c} \sqrt{E_{c} \delta}
$$

where $\bar{c} \approx 0.1$ is the numerical coefficient. In the next subsections we discuss the elastic and inelastic contributions to the variable range hopping.

\section{Hopping via elastic co-tunneling}

Considering the probability for an electron to tunnel from the site $i_{0}$ to the site $i_{N+1}$ it is convenient to put the Coulomb interaction energy at these sites to zero and count the initial, $\xi_{0}$, and final, $\xi_{N+1}$, electron energies from the Fermi level. The presence of the electrostatic disorder on the grains is modelled by the random potential $V_{i}$. The energy of the electron (hole) excitation on the site $i$ is

$$
E_{i}^{ \pm}=E_{i}^{c} \pm \mu_{i}
$$

where $\mu_{i}=V_{i}+2 \sum_{j} E_{c}^{i j} n_{j}$ is the local potential that along with the bare potential $V_{i}$ includes the potential induced by the neighboring grains. The probability $P_{e l}$ of a tunneling process via the elastic cotunneling can most easily be found for the case of the diagonal Coulomb interaction $E_{i j}^{c}=E_{i}^{c} \delta_{i j}$. We leave calculational details for the Appendix A and use here only the final result of the derivation.

The probability of the elastic cotunneling through $N$ grains can be written as

$$
P_{e l}=w g_{0}\left(\frac{\bar{\Gamma}}{\pi \bar{E}}\right)^{N} \delta\left(\xi_{N+1}-\xi_{0}\right),
$$

where the factor $w=n\left(\xi_{0}\right)\left[1-n\left(\xi_{N+1}\right)\right]$ takes into account the occupations $n\left(\xi_{0}\right)$ and $n\left(\xi_{N+1}\right)$ of the initial and final states respectively (the initial state is filled and the final one is empty). The bar in Eq. (2.105) denotes the geometrical average of the physical quantity along the tunneling path. For example, the average energy $\bar{\Gamma}$ is defined as

$$
\ln \bar{\Gamma}=\frac{1}{N+1} \sum_{k=0}^{N} \ln \Gamma_{k}
$$

where the summation extends over the tunneling path, $\Gamma_{k}=g_{k} \delta_{k}$ and $g_{k}$ is the tunneling conductance between the $k$-th and $(k+1)$-st grains. [We note that the meaning of $\Gamma_{i}$ as an escape rate from a grain does not hold in the insulating regime under consideration.] Yet, we use this notation even in the insulating sate. The energy $\bar{E}$ is the geometrical average $\ln \bar{E}=\frac{1}{N} \sum_{k=1}^{N} \ln \tilde{E}_{k}$, of the following combination of the electron and hole excitation energies

$$
\tilde{E}_{k}=2\left(1 / E_{k}^{+}+1 / E_{k}^{-}\right)^{-1} \text {. }
$$

The presence of the delta function in Eq. 2.105 reflects the fact that the tunneling process is elastic.

The form of Eq. 2.105 for the tunneling probability $P_{e l}$ corresponds to an independent sequential tunneling from grain to grain. This equation means that on average the probability falls off exponentially with the distance $s$ along the path:

$$
P_{e l} \sim \exp \left(-2 s / \xi_{e l}\right),
$$

where the dimensionless localization length $\xi_{e l}$ can be written as

$$
\xi_{e l}=\frac{2}{\ln (\bar{E} \pi / c \bar{g} \delta)} .
$$

Both the distance $s$ and the length $\xi_{e l}$ in Eqs. 2.108 2.109) are measured in the units of the grain size $a$. The numerical constant $c$ in Eq. (2.109) is equal to unity for the model of the diagonal Coulomb interaction, $E_{i j}^{c}=$ $E_{c} \delta_{i j}$. We show in Appendix A that the inclusion of the off-diagonal part of the Coulomb interaction results in a renormalization of the constant $c$ to a certain value $0.5 \lesssim c<1$.

Applying the conventional Mott-Efros-Shklovskii arguments, i.e. optimizing the full hopping probability that is proportional to

$$
\exp \left[-\left(2 s / \xi_{e l}\right)-\left(e^{2} / \tilde{\kappa} T a s\right)\right]
$$

one obtains Eq. (2.14) for the hopping conductivity with the characteristic temperature

$$
T_{0} \sim e^{2} / a \tilde{\kappa} \xi_{e l},
$$

where $\tilde{\kappa}$ is the effective dielectric constant of a granular sample, $a$ is the average grain size, and $\xi_{e l}$ is given by Eq. (2.109) (when deriving Eq. (2.111) we considered the tunneling path as nearly straight).

In the presence of a strong electric field $\mathcal{E}$, a direct application of the results of Shklovskii (1973) gives in the limit of low temperatures

$$
\frac{T}{e \xi_{e l} a} \ll \mathcal{E} \ll \frac{\sqrt{E_{c} \delta}}{e a},
$$

the following expression for the nonlinear current dependence

$$
j \sim j_{0} \exp \left[-\left(\mathcal{E}_{0} / \mathcal{E}\right)^{1 / 2}\right]
$$

where the characteristic electric field $\mathcal{E}_{0}$ is given by the expression

$$
\mathcal{E}_{0} \sim T_{0} / e a \xi_{e l}
$$


In a full analogy with the hopping conductivity in semiconductors (Shklovskii and Efros, 1988), also in granular metals inelastic processes are required to allow an electron to tunnel to a state with a higher energy. In the granular metals such processes occur due to interaction with phonons as well as due to inelastic collisions with other electrons. It is clear that these processes were not considered when deriving Eq. (2.105). Therefore, the results presented in this subsection are valid as long as the contribution of the inelastic cotunneling to the hopping conductivity can be neglected. This corresponds to the limit of low temperatures and electric fields $T$, $\mathcal{E}$ ea $\ll T_{\text {cross. }}$. Below, we present the results for the opposite limit when the inelastic processes give the main contribution to the hopping conductivity.

\section{Hopping via inelastic co-tunneling}

The inelastic cotunneling is the process when the charge is transferred by different electrons on each elementary hop. During such a process the energy transferred to a grain by the incoming electron differs from the energy taken away by the outgoing one.

As in the case of the elastic cotunneling we assume that the electron tunnels from the grain $i_{0}$ with the energy $\xi_{0}$ to the grain $i_{N+1}$ with the energy $\xi_{N+1}$. For the probability of such a tunneling process through a chain of the grains we find the following expression [see Appendix B and Ref. (Beloborodov et al., 2005c)]

$P_{i n}=\frac{w}{4 \pi T} \frac{\bar{g}^{N+1}}{\pi^{N+1}}\left[\frac{4 \pi T}{\bar{E}}\right]^{2 N} \frac{\left|\Gamma\left(N+\frac{i \Delta}{2 \pi T}\right)\right|^{2}}{\Gamma(2 N)} \exp \left(-\frac{\Delta}{2 T}\right)$,

where $\Gamma(x)$ is the Gamma function and $\Delta=\xi_{N+1}-\xi_{0}$ is the energy difference between the final and initial states. The appearance of the factor $\exp (-\Delta / 2 T)$ is consistent with the detailed balance principle. Indeed, at finite temperatures an electron can tunnel in two ways: either with an increase or with a decrease of its energy. According to the detailed balance principle the ratio of such probabilities must be $\exp (\Delta / T)$, that is indeed the case for the function $P_{i n},(2.114$, since apart from the factor $\exp (-\Delta / 2 T)$ the rest of the equation is even in $\Delta$.

In order to obtain the expression for the hopping conductivity one has to optimize Eq. (2.114) with respect to the hopping distance $N$ under the constraint

$$
\text { Na } \tilde{\kappa} \Delta / e^{2} \sim 1,
$$

that follows from the ES expression for the density of the ground states (2.99). Optimization of Eq. (2.114) is a bit more involved procedure than the standard derivation of Mott-Efros-Shklovskii law based on the Gibbs energy distribution function (see Appendix B). Nevertheless, it leads to essentially the same result, i.e. the ES law, Eq. (2.14), where the characteristic temperature $T_{0}(T)$ takes the form

$$
T_{0}(T) \sim e^{2} / a \tilde{\kappa} \xi_{i n}(T),
$$

with a dimensionless weakly temperature dependent localization length $\xi_{\text {in }}(T)$,

$$
\xi_{\text {in }}(T)=\frac{2}{\ln \left[\bar{E}^{2} / 16 c \pi T^{2} \bar{g}\right]},
$$

where the coefficient $c$ equals unity for a model with a diagonal Coulomb interaction matrix.

Comparing the characteristic lengths $\xi_{e l}(T)$ and $\xi_{\text {in }}(T)$ for the elastic and inelastic processes, respectively, we see easily that $\xi_{e l}(T)>\xi_{\text {in }}(T)$ at temperatures $T<T_{\text {cross }}$, where the temperature $T_{\text {cross }}$ is given by Eq. (2.103). This means that the mechanism of the inelastic scattering is more efficient at not very low temperatures exceeding $T_{\text {cross }}$.

As in the case of the elastic cotunneling, the long range electrostatic interaction results in the reduction of the coefficient to some $1 / 4 \lesssim c<1$.

At zero temperature, Equation (2.114) for the inelastic probability $P_{i n}$ can be simplified for $\Delta<0$ to the form

$$
P_{\text {in }}(T=0)=\frac{w 2^{2 N} \pi}{(2 N-1) !} \frac{|\Delta|^{2 N-1}}{\bar{E}^{2 N}}\left(\frac{\bar{g}}{\pi}\right)^{N+1},
$$

while for $\Delta>0$, Eq. (2.114) gives zero since the electron tunneling process with an increase of the electron energy is prohibited at zero temperature.

In order to obtain the hopping conductivity in the regime of a strong electric field $\mathcal{E}$ and low temperature, we use, following Shklovskii (1973), the condition (2.115) that defines the distance to the first available electron site within the energy shell $\Delta$. We use also the equation

$$
e \mathcal{E} r \sim \Delta
$$

that relates $\Delta$ to the electric field $\mathcal{E}$, and the fact that the distance between the initial and final tunneling sites is $r \sim N a$. Equations (2.115) and (2.119) allow us to determine the quantities $\Delta \sim \sqrt{\mathcal{E} e^{3} / \tilde{\kappa}}$ and $N \sim \sqrt{e / \tilde{\kappa} \mathcal{E} a^{2}}$. Inserting their values into Eq. (2.118) we come to the following expression for the current

$$
j \sim j_{0} \exp \left[-\left(\mathcal{E}_{0} / \mathcal{E}\right)^{1 / 2}\right] .
$$

where the characteristic electric field $\mathcal{E}_{0}$ is a weak function of the applied field $\mathcal{E}$

$$
\mathcal{E}_{0}(\mathcal{E}) \sim \frac{e}{\tilde{\kappa} a^{2}} \ln ^{2}\left[\bar{E}^{2} / e^{2} \mathcal{E}^{2} a^{2} \bar{g}\right]
$$

The results obtained in the insulating regime show that the hopping conductivity is the main mechanism of transport in the low temperature regime. The temperature dependence of the conductivity is determined by the Efros-Shklovskii law, Eq. (2.14), and is similar to the one in amorphous semiconductors. The exponential dependence of the current on a strong electric field is given by Eqs. (2.112) and (2.120). Again, this is the same dependence as in the amorphous semiconductors. Of course, the characteristic temperature $T_{0}$ and electric field $\mathcal{E}_{0}$ are model dependent. 
The theory for the variable range hopping ( $\mathrm{VRH})$ via virtual electron tunneling through many grains is based on the assumption that the hopping length $r^{*}$ exceeds the size of a single grain $a$. This hopping length $r^{*}$ should be found minimizing the exponent in Eq. (2.110). From this condition we see that the characteristic length $r^{*}(T)$ equals

$$
r^{*}(T)=\sqrt{\frac{e^{2} \xi a}{2 T \bar{\kappa}}},
$$

where the dimensionless localization length $\xi$ is given by either Eq. (2.109) or Eq. (2.117), and that it decreases with increasing the temperature. At temperatures $\tilde{T} \sim$ $e^{2} \xi / 2 a \tilde{\kappa}$ the hopping length becomes of the order of the grain size, $r^{*}(\tilde{T}) \sim a$. At such distances the VRH picture does not work anymore because the electrons hope between the neighboring grains only. This means that at comparatively high temperatures $\tilde{T} \ll T \ll \Delta_{M}$ one should use the Arrhenius law, Eq. (2.13) instead of the Efros-Shklovskii law, Eq. (2.14). Such a crossover has been observed experimentally (Tran et al., 2005).

Thus, we have obtained a remarkable result: Even though the array of the grains may look very regular, i.e. have a periodic structure and equal size and shape of the grains, it is almost inevitable that at low temperatures the main mechanism of the conduction is the variable range electron hopping and the conductivity is determined by Eq. (2.14) as if the system were amorphous. This concerns also other formulae like Eqs. 2.112 and (2.120).

\section{ARRAYS COMPOSED OF SUPERCONDUCTING GRAINS}

In this Section we consider properties of granular materials consisting of superconducting particles. As in the Section III devoted to study of normal metals we start our presentation in the next subsection with a qualitative discussion of the most interesting effects that occur in these systems. A quantitative analysis of these effects will be given in the subsequent subsections.

\section{A. General properties of granular superconductors}

\section{Single grain}

A granular superconductor, similarly to a granular metal, can be viewed as an array of superconducting granules coupled via electron tunneling. Superconducting properties of an array in many ways are determined by the properties of granules which it is formed of. For this reason we begin our discussion with reviewing properties of superconductivity in a single small isolated grain.

This question was first addressed by Anderson (Anderson, 1959) who realized that the s-wave superconductivity is almost insensitive to disorder of general kind as long as it does not break the time reversal invariance. This includes, in particular, the diffusive scattering by the grain boundaries that is in many ways similar to the scattering by potential impurities in the bulk. As long as the time reversal invariance is not broken, one can choose the basis of exact eigenfunctions and then apply the Bardeen-Cooper-Schrieffer (BCS) theory in the usual way. The critical temperature can still be expressed via the BCS effective coupling constant and the density of states in the vicinity of the chemical potential. The BCS coupling constant does not change considerably when changing the basis and the average density of states is not sensitive to disorder. Thus, one concludes (Anderson, 1959) that the critical temperature of the single grain has to be close to the bulk value.

Anderson has also pointed out that these arguments hold even for small grains provided the average distance between the energy levels $\delta$, Eq. (1.3) is still smaller than the superconducting gap $\Delta$, in the bulk Anderson, 1959). As soon as the energy level spacing $\delta$ reaches the superconducting gap, the conventional BCS theory is no longer applicable.

This fact can easily be understood from the BCS equation for the order parameter that can be written as

$$
\Delta=\delta \lambda_{0} T \sum_{\omega} \sum_{\xi_{k}} \frac{\Delta}{\omega^{2}+\xi_{k}^{2}+|\Delta|^{2}}
$$

where $\lambda_{0}$ is the standard dimensionless constant describing the attraction between the electrons, $\omega=$ $2 \pi T(n+1 / 2)$ are Matsubara frequencies and $\delta$ is the average mean energy level spacing in a grain determined by Eq. (1.3). The discrete variable $\xi_{k}$ equals

$$
\xi_{k}=\epsilon_{k}-\mu
$$

where $\epsilon_{k}$ are energies of the electron states in the grains and $\mu$ is the chemical potential.

If the grain size is very large, such that the mean level spacing $\delta$ is much smaller than the superconducting critical temperature $T_{c 0}$, the sum over the states in Eq. (3.1) can be replaced by the integral

$$
\delta \sum_{\xi_{k}} \rightarrow \int_{-\infty}^{\infty} d \xi
$$

and we come to the conventional BCS equation.

At the same time, it is clear from Eq. (3.1) that a finite difference between the energy levels $\xi_{k}$ plays a similar role as a finite temperature, namely, it reduces the superconducting gap $|\Delta|$. As soon as the mean level spacing $\delta$ exceeds $T_{c 0}$ (or $\Delta(T=0)$ for the bulk superconductors), the superconducting gap $|\Delta|$ vanishes. To be more precise, it remains finite due to fluctuations but its value is sufficiently reduced and in this regime the grain cannot be considered as a "true" superconductor. Nevertheless, the superconducting pairing is 
still present manifesting, for example, in the parity effect, (von Delft et al., 1996; Matveev and Larkin, 1997; Smith and Ambegaokar, 1996).

The Anderson theory agrees well with experiments. In particular, electron spectrum of ultrasmall superconducting $A l$ grains was studied by Black et al. (1996); Davidović and Tinkham (1999); Ralph et al. (1995) where a well defined superconducting gap was shown to survive down to grain sizes $\sim 10 \mathrm{~nm}$, while smaller grains $\leq 5 \mathrm{~nm}$ did not show any superconducting features in their electron spectrum.

It is worth noting that, while in the diffusive/chaotic grains the density of states in the vicinity of the chemical potential indeed well agrees with the corresponding bulk values, this is not necessarily the case for the ballistic grains possessing certain geometric symmetries. For example, in a spherical grain one naturally expects that the energy levels are strongly degenerate. This may change the density of states on the Fermi level significantly resulting in a considerable change of the transition temperature. The possibility to utilize the enhanced density of states that may appear from the orbital degeneracy in order to increase the single grain critical temperature was recently suggested in Refs. (Ovchinnikov and Kresin, 2005a b).

Although all these effects are very interesting, in this review we follow our line of studying models with parameters that can easily be achieved in existing granular materials. So, we assume everywhere that granules are sufficiently large to maintain the local superconductivity within each grain, i.e. that the Anderson criterion is satisfied

$$
\delta \ll \Delta
$$

We assume also that the grains are diffusive or do not have an ideal shape like spheres or cubes, which excludes the possibility of an accidental degeneracy of the electron spectrum.

As a final remark in our discussion of superconductivity in a single grain, we would like to point out that the thermodynamics of a single grain is not affected by the presence of the Coulomb interaction as long as the latter can be expressed in a standard way through the operator of the total electron number. The reason is that the number of particles in an isolated grain is conserved and thus, the Coulomb term in the Hamiltonian commutes with the rest of the Hamiltonian and does not influence the thermodynamic quantities.

At the same time, phase fluctuations of the order parameter $\Delta(\tau)$

$$
\Delta(\tau)=\left|\Delta_{0}\right| \exp [2 i \varphi(\tau)]
$$

are very sensitive to the presence of the charging term, Eq. (2.23). These fluctuations can be conveniently characterized by the correlation function $\Pi_{s}(\tau)$

$$
\Pi_{s}(\tau)=\langle\exp (2 i[\varphi(\tau)-\varphi(0)])\rangle,
$$

where the averaging should be performed over the states of the Hamiltonian including the Coulomb interaction, Eq. (2.23).

Actually, the average over the states of the Hamiltonian in Eq. (3.6) reduces to an average with the action $S_{c}$, Eq. (2.56), and can rather easily be calculated (Efetov, 1980) leading to the result at $T=0$

$$
\Pi_{s}(\tau)=\exp \left(-4 E_{c} \tau\right)
$$

where $E_{c}=E_{i i}$ is the Coulomb energy of a single grain and a factor 4 appears because of the doubled Cooper pair charge. Equation (3.7) is written for the imaginary time $\tau$. Changing to the real time, $\tau \rightarrow i t$, one can conclude that the correlation function $\Pi_{s}(\tau)$ oscillates in time. Although the exponential form of the function $\Pi_{s}(\tau)$ does not lead to changes in the thermodynamics, it is crucial for the problem of the coherence in an array of many grains.

\section{Macroscopic superconductivity}

Having listed the superconducting properties of a single grain we turn now to discussing the superconductivity in the whole sample. In order to simplify the presentation we first restrict ourselves to the zero temperature case.

It is clear that an array with sufficiently strongly coupled grains should be able to maintain the superconducting coherence in a whole sample because the coupling reduces the phase fluctuations. On the contrary, in the opposite limit of a weak coupling, one expects that the strong Coulomb interaction should lead to the Coulomb blockade of the Cooper pairs in analogy with the Coulomb blockade of electrons in granular metals in the low coupling regime.

In order to quantify this intuitive statement Abeles (Abeles, 1977), following the earlier idea of Anderson (Anderson, 1964), suggested to compare the energy of the Josephson coupling of the neighboring grains with the Coulomb energy. Indeed, the Josephson coupling tends to lock the phases of the neighboring grains and to delocalize the Cooper pairs, while the Coulomb interaction tends to localize the Cooper pairs and thus enhance the quantum phase fluctuations.

Comparing the Josephson energy $J$,

$$
J=\pi g \Delta / 2
$$

with the Coulomb energy one would come then to the conclusion that samples with $g>g_{s} \sim E_{c} / \Delta$ should be superconductors, while those with $g<g_{s}$ - insulators. This result was later derived within a model for a superconducting granular array including both Coulomb and Josephson interactions in a number of theoretical works (Efetov, 1980; McLean and Stephen, 1979; Simanek, 1979) using different methods. We will review the main results of these studies in the Sec. III.C 


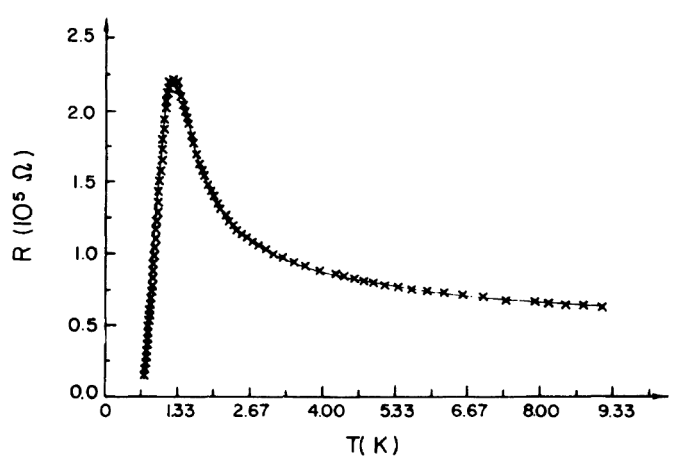

FIG. 12 Resistance as a function of temperature of one of the granular samples of Shapira and Deutscher (1983). In spite of the fact that the sample exhibits strong insulating behavior in the intermediate temperature range it finally turns to the superconducting state.

The Anderson-Abeles criterion predicts that the superconductivity may survive even in such samples that would be insulators if they were made from normal grains under equivalent conditions. Indeed, for samples with a small ratio $E_{c} / \Delta \ll 1$, there is a parametrically large interval of conductances $E_{c} / \Delta \ll g \ll 1$ where superconductivity should exist in spite of the fact that the corresponding normal array would be an insulator.

Qualitatively, this prediction was supported by experiments (Shapira and Deutscher, 1983) where some superconducting samples were found to show clear insulating behavior above the critical temperature. (We note, however, that the ratio $E_{c} / \Delta$ in the samples used in this experiment was certainly large, this fact, as we will show below, may be explained via renormalization of the Coulomb energy). The corresponding dependence of the resistance on temperature according to Shapira and Deutscher (1983) is shown in Fig. 12

However, the Anderson-Abeles criterion was found later to be in conflict with many other experiments. After the pioneering works (Jaeger et al., 1989, 1986; Orr et al., 1985, 1986) it became clear that the boundary between the superconducting and insulating states at $T=0$ is determined by the normal state conductance of the film rather then by the ratio of the Josephson and Coulomb energies.

This fact is illustrated by the experimental resistivity temperature dependencies of samples with different thicknesses shown in Fig. 13] $\mathrm{Ga}$ and $\mathrm{Pb}$ ultrathin films. One can see that there exists a critical value of the normal state resistance $R_{0}$ close to the value of quantum resistance $h /(2 e)^{2} \approx 6.4 \mathrm{k} \Omega$ such that samples with resistance $R<R_{0}$ eventually become superconducting under temperature decrease, while those with $R>R_{0}$ show tendency to the insulating behavior. Such a behavior turned out to be generic and was observed in a number of granular (as well as on homogeneously disordered, (Frvdman et al., 2002; Liu et al., 1993; Marković et al., 1999)) films made from different materials.

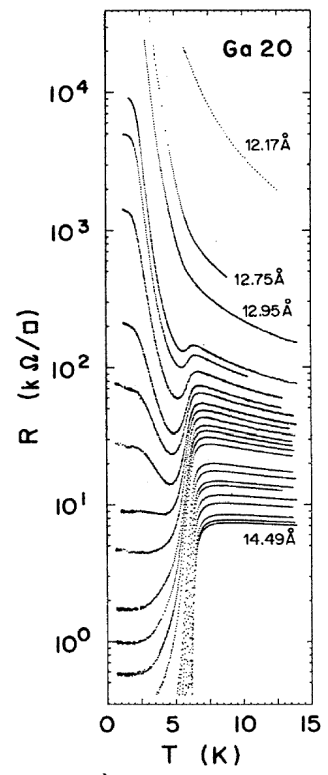

a)

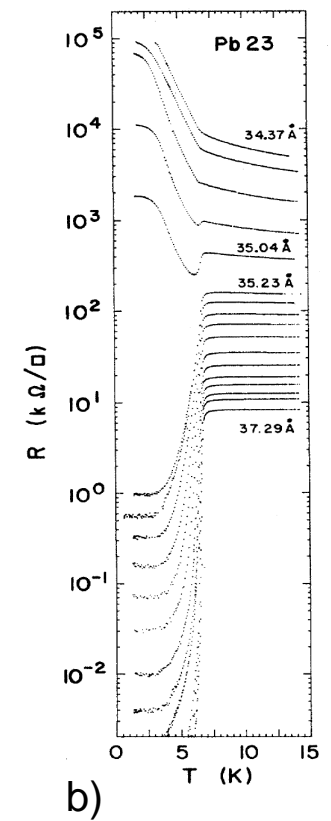

FIG. 13 Temperature dependence of resistivity of granular (a) $\mathrm{Ga}$ and (b) $\mathrm{Pb}$ ultra thin films according to Jaeger et al. (1989). Different curves correspond to the films with different normal state resistances that is tuned by the sample thickness. Low temperature state is controlled by the film normal state resistance.

In this respect, the superconductor-insulator transition in thin granular films resembles the analogous transition in a resistively shunted Josephson junction (Bulgadaev, 1984; Schmid, 1983) where the state of the system is known to be controlled by the value of the shunt resistance only, no matter what the ratio of the Coulomb and Josephson energies is (for review see Schön and Zaikin $(1990)$ ). The resistive shunt in this approach is included via the Caldeira-Leggett approach (Caldeira and Leggett, 1981, 1983) that allows to take into account the dissipative processes on the quantum mechanical level. Similar approach based on the inclusion of the phenomenological dissipative term that models the resistive coupling of the grains was applied to the granular system in order to resolve the disagreement with experiment (Chakravarty et al., 1986; Fisher, 1986; Simánek and Brown, 1986). It was found that in the limit $E_{C} / J>1$, in agreement with experiment, the boundary between the superconducting and insulating states is controlled by the resistance of the shunts only rather than by the ratio of $E_{C} / J$.

However, the presence of the phenomenological resistive coupling between the grains is rather difficult to justify, especially in the limit of low temperatures where all quasiparticles are frozen out. This problem was finally resolved by Chakravarty et al. (1987) on the basis of the model that took into account the direct electron tunneling processes between the grains in addition to the Josephson couplings. The phase diagram obtained 


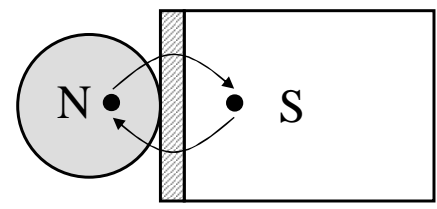

FIG. 14 Illustration of the renormalization of the Coulomb energy of a normal grain placed in a contact with a bulk superconductor: Due to the virtual electron tunnelling processes the Coulomb energy of a normal grain $E_{c}$ is renormalized down to the values $\tilde{E}_{c}=\Delta / 2 g$. The conductance of the contact is supposed to be large $g \gg 1$ and the initial Coulomb energy is assumed to be larger than the superconducting gap $\Delta$.

in this approach turned out to be similar to the one obtained within the "dissipative" model. At the same time, the considered model did not rely on any phenomenological assumptions and the virtual intergrain tunneling processes were well described in terms of the original tunneling Hamiltonian.

According to the approach of Chakravarty et al. (1987), the originally strong Coulomb interaction is reduced by the electron tunneling to other grains. This renormalization of the Coulomb energy can be well understood on example of a simplified model of a normal grain placed in a contact with the bulk superconductor shown in Fig. 14 It is clear that the Coulomb charging energy is reduced due to the possibility for an electron to be present virtually in the superconductor. Actually, this renormalization is nothing but the screening of the Coulomb interaction by free charges, which is usual for metals.

The value of the screened charging energy in the case of a strong coupling between the grains, $g \gg 1$, and strong Coulomb interaction, $E_{c} \gg \Delta$ turns out to be $\sim \Delta / g$; and what is remarkable, is independent on the original energy $E_{c}$. Similar result holds for the superconducting grain as well. We see that in the limit $g \gg 1$ the Josephson energy $J \sim g \Delta$ is always larger than the effective Coulomb energy $\Delta / g$ meaning that a sample with $g \gg 1$ should always be a superconductor at sufficiently low temperature (Eckern et al., 1984; Larkin and Ovchinnikov, 1983). Another, important conclusion is that the superconductor-insulator transition occurs at $g \sim 1$, which is in agreement with the experiments. The phase diagram obtained by Chakravarty et al. (1987) is presented in Fig. 115.

The fact that an array consisting of strongly coupled superconducting grains $(g \gg 1)$ has to be in a superconducting state at sufficiently low temperatures can be understood from a somewhat more general consideration. In the strong coupling regime, the granular system resembles the homogeneously disordered system. According to Anderson (Anderson, 1959), within the accuracy of the mean field BCS theory, the thermodynamics of such a system is essentially the same as that of a pure bulk elec-

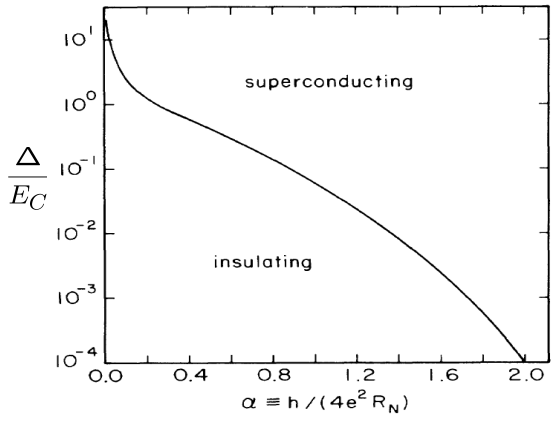

FIG. 15 Zero temperature phase diagram of the granular superconducting array according to Chakravartv et al. (1987). Dimensionless parameter $\alpha$ is related to the conductance $g$ as $\alpha=\pi g$.

tron system. This means that the granular sample turns to the superconducting state immediately after the superconducting gap appears locally in each grain.

Thus, the insulating state where the superconductivity exists locally, while being absent in the whole sample, may be obtained only beyond the BCS approximation. Corrections to the BCS theory may come from the Coulomb interaction and superconducting fluctuations but they are expected to be small in the limit $g \gg 1$. The fact that the corrections due to the Coulomb interaction are small in normal metals in the limit $g \gg 1$ has been demonstrated in Sec. III This result allows to come to the conclusion that at $g \gg 1$ the BCS theory is a good starting point for description of the granular system.

This in particular means that the critical temperature of a good conducting granular sample $(g \gg 1)$ is approximately given by the single grain BCS critical temperature and that the corrections to $T_{c}$ can be studied by means of the perturbation theory in $1 / \mathrm{g}$. In the case of homogeneously disordered films such a perturbative approach to calculation of $T_{c}$ is well known (Finkelstein, 1987; Maekawa et al., 1983; Ovchinnikov, 1973). Analogously, one can consider the corrections to the critical temperature in granular systems using a perturbation theory in the inverse conductance. We discuss this approach and results obtained in Sec. III.D

In the opposite limit, $g \ll 1$, the critical temperature can be found from an effective model that includes the Josephson and Coulomb interaction only. As the probability of the tunneling from grain to grain is small, the effect of the screening due to the electron tunneling can be neglected. In particular, in the simplest case of a very small charging energy, one can estimate the critical temperature $T_{c}$ by comparing the Josephson energy $J \sim \Delta g$ with the temperature, which leads to the estimate $T_{c} \sim \Delta g$. We will consider this question within a mean field approximation in more detail in the next subsection. 


\section{Granular superconductor in a magnetic field}

Now we present new features in the behavior of a granular array that manifest in an applied magnetic field. It turns out that magnetic properties of granular metals are very different from those of the corresponding bulk systems even in the limit of large tunneling conductances, $g \gg 1$. In particular, there appears a new characteristic conductance value

$$
g^{*} \approx 0.16(a \delta)^{-1} \sqrt{\Delta_{0} D}
$$

where $\Delta_{0}$ is the superconducting order parameter for a single grain at zero magnetic field and zero temperature and $D$ is the diffusion coefficient in the grain.

The critical magnetic field $H_{c}$ of samples with $g<g^{*}$ is close to the critical field $H_{c}^{\text {gr }}$ of a single grain, while for samples with $g>g^{*}$, it is close to the one in a homogeneously disordered metals with the diffusion coefficient $D_{\text {eff }}=g \delta a^{2}$ (c.f. Eqs. 2.2 2.3). The latter is the effective diffusion coefficient of the granular array. We note that the conductance $g^{*}$ corresponds to the well conducting samples. Indeed, expressing $g^{*}$ in terms of the Thouless energy $E_{T h} \sim D / a^{2}$ we obtain

$$
g^{*} \sim\left(\frac{E_{T h} \Delta_{0}}{\delta^{2}}\right)^{1 / 2} \gg 1 .
$$

The dependence of the critical magnetic field on the sample conductance and temperature will be considered in detail in Sec. III.C Here we will only show how the new conductance scale (3.9) appears: The critical magnetic field $H_{c}^{\mathrm{gr}}$ destroying the superconductivity in a single grain of a spherical form due to the orbital mechanism is given by (Larkin, 1965) (the derivation is presented in Sec. III.C)

$$
H_{c}^{\mathrm{gr}}=\frac{c}{e} \sqrt{\frac{5 \Delta_{0}}{2 R^{2} D}}
$$

where the grain radius $R$ is related to to the period of a cubic granular array as $R=a / 2$. At sufficiently strong coupling the critical field of the sample, on the contrary, has to be given by the bulk value expressed via the effective diffusion coefficient:

$$
H_{c}^{\mathrm{bulk}}=\frac{\Phi_{0}}{2 \pi} \frac{\Delta_{0}}{D_{e f f}},
$$

where $\Phi_{0}=h c / 2 e$ is the flux quantum. Comparing Eqs. 3.11) and (3.12) one can see that the two critical magnetic fields $H_{c}^{\mathrm{gr}}$ and $H_{c}^{\mathrm{bulk}}$ become of the same order at tunneling conductances $g \simeq g^{*}$.

The orbital mechanism of the destruction of the superconductivity leading to Eqs. (3.11) and (3.12) is dominant provided the grains are not very small. However, in the limit of very small sizes of the grain, the Zeeman mechanism of the destruction can become more important (Beloborodov et al., 2000; Kee et al., 1998;
Tinkham, 1996) (everywhere in this review we consider the $s$-wave singlet pairing).

If the Zeeman mechanism dominates the orbital one the critical magnetic field $H_{c}^{z}$ is given by the Clogston value

$$
H_{c}^{z}=\Delta_{0} / \sqrt{2} \mu_{B}
$$

where $\mu_{B}=e \hbar / 2 m c$ is the Bohr magneton, Clogston, 1960). We note that the phase transition between the superconducting and normal states is in this case of the first order.

In the opposite limit, when the superconductivity is destroyed mainly by the orbital mechanism, the Zeeman splitting has no dramatic effect on the phase diagram. Comparing the orbital and Zeeman critical magnetic fields, Eqs. 3.11 3.13 one can see that the latter can be neglected as long as the grain is not too small $a>a_{c}$ with the critical grain size $a_{c}$ given by

$$
a_{c} \approx \sqrt{\frac{5}{D \Delta_{0} m^{2}}} \sim \frac{1}{p_{0}} \sqrt{\frac{E_{T h}}{\Delta_{0}}} .
$$

Throughout our review we assume that the grains are not too small such that the phase transition within a singe grain remains of the second order. Of course, we speak about the phase transition within the mean field approximation. Fluctuations of the order parameter $\Delta$ smear the transition in the isolated grain.

\section{Transport properties of granular superconductors}

Experimentally observed dependencies of the resistivity of the granular superconductors on temperature and applied magnetic field are very reach and all details are currently far from being well theoretically understood. In our review we discuss only briefly the general transport properties touching in detail only the latest developments related to the low temperature magnetoresistance in granular superconductors. A broader discussion of theoretical advances in understanding the transport properties of granular superconductors (networks of Josephson junctions) can be found in Ref. (Fazio and van der Zant, 2001).

In the absence of the magnetic field, the dependence of the resistivity of a granular superconductor on temperature is typically non-monotonic. One of the examples is shown in Fig. 12. Decreasing the temperature first leads to a growth of the resistivity, which is due to the enhancement of the Coulomb correlations that suppress the current. However, the resistivity starts decreasing closer to the superconducting transition temperature. In this region the conductivity is enhanced due to the fluctuation contribution of the Cooper pairs (Aslamazov and Larkin, 1968).

Granular films with the normal state resistance close to the critical value $R_{0} \sim 6.4 k \Omega$ exhibit often a more complicated reentrant behavior as it is seen in Fig. 13 


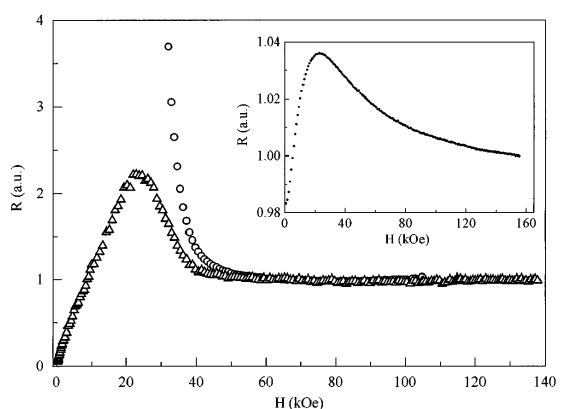

FIG. 16 Low temperature resistance of $3 d \mathrm{Al}$ granular samples as a function of magnetic field at low temperatures, $T \ll T_{c}$, (Gerber et al., 1997). The two curves are shown for two samples with different normal temperature resistance. The insert shows much less pronounce peak in the resistance at higher temperatures, $T-T_{c} \ll T_{c}$.

The resistance of such samples increases again after a drop associated with the fluctuating conductivity. This effect can be a consequence of the competition between the conductance increase due to the fluctuating Cooper pairs and the freezing of the excitations due to the opening of the superconducting gap in the density of states.

The systematic theoretical description of this phenomenon is not available yet. We can only note that the reentrant behavior of the conductivity seems to be a consequence of the granularity. Homogeneous films made from the same material having no (or less pronounced) granular structure do not show such a behavior (Liu et al., 1993).

Magnetic field applied to a granular system brings an extra control parameter allowing to change the resistivity and to tune the state of the granular system. In particular, sufficiently strong magnetic field can always destroy the superconductivity even at low temperatures.

What is interesting, the resistance of a granular superconductor has a non-monotonic dependence on the applied magnetic field at low temperatures. As an example we present results of a measurement on $A l$ granular from Gerber et al. (1997). The samples used by Gerber et al. (1997) were three dimensional and monodisperse with a typical diameter of the grains $\approx$ $120 \mathrm{~A}$. A surprising feature of the experimental curves is that there is a region with a negative magnetoresistance and a pronounced peak in the resistivity at the magnetic field of several Tesla is seen. Only at extremely strong fields, $H>6 T$, the resistivity is almost independent of the field.

The non-monotonic behavior of resistivity can be understood in terms of the corrections to the conductivity due to the superconducting fluctuations (Beloborodov and Efetov, 1999; Beloborodov et al., 2000). In the vicinity of the phase transition the resistivity decreases due to the opening of the additional transport channel via fluctuating Cooper pairs. This is what is called the Aslmazov-Larkin conductivity correction (Aslamazov and Larkin, 1968).
We note that this correction gives always a positive contribution to the conductivity and thus cannot explain the negative magnetoresistance at higher fields. However, at stronger fields another conductivity correction that appears as a result of the reduction of the density of states due to fluctuating Cooper pairs begins to dominate electron transport (Beloborodov and Efetov, 1999). As a result, in this regime the conductivity becomes lower than that in the normal metal approaching the latter only in the limit $H \gg H_{c}$, where all the superconducting fluctuations are completely suppressed by the magnetic field. We discuss the magnetoresistance of granular superconductors in more detail in Sec. III.E

Changing the magnetic field in the limit $T \rightarrow 0$ one can cross the boundary between the superconducting and metallic/insulating states and investigate the transport around the zero temperature (quantum) phase transition. The corresponding transition point at $T=0$ is referred as quantum critical point (Sachdev, 2001; Sondhi et al., 1997).

Quantum phase transitions are analogous to the classical phase transitions with the differences that at $T \rightarrow 0$ the system has been described not only via the characteristic spatial (coherence length) $\xi$ but also via the characteristic time scale $\tau$. Both $\tau$ and $\xi$, as in the case of classical transitions, are assumed to scale as powers of the parameter $\delta$ that controls the closeness of the system to the quantum critical point.

Scaling ideas were applied to the problem of the superconductor to insulator transition in Refs. (Fisher, 1990; Fisher et al., 1990) that gave a substantial impact on the field. Scaling expressions for the resistivity seem to work well for many samples. However, one of the central prediction of Refs. Fisher, 1990; Fisher et al., 1990) - the universal conductance at the superconductor to insulator transition is not supported by all experiments (Chervenak and J. M. Valles, 2000; Goldman and Markovió, 1998; van der Zant et al., 1992).

Of course, the scaling approach to the resistivity behavior is not restricted to the granular systems and, on the contrary, homogeneously disordered films are much more suitable candidates for experimental study of such phenomena. For this reason we will not further touch this question referring the reader to Refs. (Fazio and van der Zant, 2001; Goldman and Marković, 1998; Sondhi et al., 1997).

\section{B. Phase diagram of granular superconductors}

In this section we discuss the phase diagram of granular superconductors in the absence of the magnetic field. We will mainly concentrate on the mean field approach and will not touch the critical behavior in the very vicinity of the phase transition and we will not discuss in detail the superconductor- insulator transition. The latter phenomenon relates to a wide class of dis- 
ordered superconductors and represents the field of research on its own. For reviews on this subject we refer the reader to Refs. (Fazio and van der Zant, 2001; Goldman and Marković, 1998; Larkin, 1999).

The arrays of the superconducting grains can be conveniently described using a phase functional analogous to the functional $S[\varphi]$ derived in Chapter $\amalg$ for the normal granular metals. In the next subsection we introduce such a functional.

\section{Phase functional for the granular superconductors}

Description of a granular superconductor in terms of the phase action is very similar to that used for the normal metals and the derivation can rather simply be extended to include the superconducting order parameter. In order to simplify the discussion we consider the limit of low temperatures $T \ll T_{c 0}$, where $T_{c 0}$ is the critical temperature in the bulk in the BCS approximation. We assume that the superconducting gap is still smaller than the Thouless energy, Eq. (1.5),

$$
\Delta \ll E_{t h},
$$

which allows us to consider a single grain as zerodimensional. Another way to write this inequality is

$$
\xi_{\text {eff }} \gg a
$$

where $\xi_{\text {eff }}=\sqrt{\xi_{0} l}, \xi_{0}=v_{F} / T_{c 0}$, and $l$ is the mean free path.

The derivation of the action $S[\varphi]$ can be carried out in the same way as in Chapter III decoupling the Coulomb interaction by an auxiliary field $V_{i}$ and the making the gauge transformation. The phase $\varphi$ is determined by Eqs. 2.44-2.47). Expanding the action in the tunneling amplitude $t_{i j}$ up to the second order we obtain the action $S[\varphi]$ in the form of a sum of three terms

$$
S[\varphi]=S_{c}[\varphi]+S_{t s}[\varphi]+S_{J}[\varphi]
$$

The first term $S_{c}[\varphi]$ stands for the charging energy and is given by Eq. (2.56). This means that the charging energy of the superconducting grain is the same as that of the normal one provided the same charge is placed onto the grains.

The second term $S_{t s}[\varphi]$ can be written in the form of Eq. (2.57) but with another function $\alpha_{s}(\tau)$ instead of $\alpha(\tau)$, Eq. (2.58). In the low temperature limit this function takes the form (Eckern et al., 1984)

$$
\alpha_{s}(\tau)=\frac{1}{\pi^{2}} \Delta^{2} K_{1}^{2}(\Delta|\tau|)
$$

where $K_{1}(x)$ is the modified Bessel function. This difference between the functions $\alpha(\tau)$, Eq. (2.58), and $\alpha_{s}(\tau)$ is very important. The function $\alpha_{s}(\tau)$ (3.18) decays exponentially when increasing $\tau$, while the functions $\alpha(\tau)$ decays only algebraically at large $\tau$. The good convergence of the kernel (3.18) allows us to expand the term
$S_{s t}$, Eqs. 2.57 3.18 in powers of $\tau-\tau^{\prime}$ and to write the term $S_{t s}$ in a simplified form

$$
S_{t s}=\frac{3 \pi g}{64 \Delta} \sum_{<i j>} \int_{0}^{\beta} \dot{\varphi}_{i j}^{2}(\tau) d \tau
$$

which should be correct for $\tau \gg \Delta^{-1}$. Only the third term $S_{J}$ in Eq. (3.17) is new as compared with normal metals; it describes the Josephson coupling between the grains and in the limit $\tau \gg \Delta^{-1}$ can be written as

$$
S_{J}=-\frac{1}{2} \sum_{\langle i j\rangle} \int_{0}^{\beta} J_{i j} \cos \left(2\left[\varphi_{i}(\tau)-\varphi_{j}(\tau)\right]\right) d \tau
$$

The sum in Eq. (3.20) should be performed over the nearest neighbors $i, j$ and $J=J_{i j}$ for these values of $i, j$ is determined by Eq. (3.8).

Equations 3.17 2.56, 2.57 3.18 3.20 3.8 describe completely the granular superconductors in the low temperature limit. They can easily be extended to higher temperatures by writing proper expressions for the functions $\alpha_{s}(\tau)$ and $J_{i j}$. Trying to present the physical picture in the simplest way we do not consider here these complications.

The action $S[\varphi]$ contains only the phases $\varphi$, whereas fluctuations of the modulus of the order parameter are neglected. This is justified at low temperatures provided the inequality (3.4) is fulfilled. A microscopic derivation of the action $S_{c}+S_{J}$ was originally done using another approach in Efetov (1980). It was also written phenomenologically in Simanek (1979) assuming however that the variable $\varphi$ varied from $-\infty$ to $\infty$. The existence of the term $S_{t s}$ was realized later (Eckern et al., 1984) and used for a system of Josephson junctions by Chakravartv et al. (1987).

Assuming that the most important are large times $\tau \gg$ $\Delta^{-1}$ we use in the subsequent calculations Eq. (3.19) for the term $S_{t s}$. Then, denoting

$$
S_{0}[\varphi]=S_{c}[\varphi]+S_{t s}[\varphi]
$$

we write the quadratic form $S_{0}[\varphi]$ as

$$
S_{0}[\varphi]=\frac{1}{4} \sum_{i, j} \int\left(\tilde{E}_{c}^{-1}\right)_{i j} \dot{\varphi}_{i}(\tau) \dot{\varphi}_{j}(\tau) d \tau,
$$

where

$$
\tilde{E}_{c}(\mathbf{q})=\frac{1}{2}\left[C(\mathbf{q}) / e^{2}+(3 \pi g / 16 \Delta) \lambda_{\mathbf{q}}\right]^{-1}
$$

and $\lambda_{\mathbf{q}}$ is to be taken from Eq. (2.30). The function $E(\mathbf{q})$ is the Fourier transform of the function $E_{i j}$ and $\mathbf{q}$ is quasimomentum. When integrating over the phases $\varphi$, one should remember about the winding numbers. Looking at Eq. (3.23) one can understand that the term $S_{t s}$, Eqs. 2.57 3.18), leads to a screening of the Coulomb interactions. 


\section{Mean field approximation}

In spite of many simplifications, the action

$$
S[\varphi]=S_{0}[\varphi]+S_{J}[\varphi]
$$

is still complicated and it is not possible to describe the system without making approximations. A common way to understand, at least qualitatively, the properties of a model is to develop a mean field theory. Following this approach one should replace the initial Hamiltonian (action) by a simplified one and determine parameters of the effective model self-consistently.

Now we will consider the action $S[\varphi]$, Eq. (3.24) in the mean field approximation. Following Efetov (1980) we make in the action $S_{J}$, Eq. (3.20), the following replacement

$$
\sum_{\langle i, j\rangle} J_{i j} \cos \left[2\left(\varphi_{i}-\varphi_{j}\right)\right] \rightarrow J z\langle\cos 2 \varphi\rangle_{M F} \sum_{j} \cos 2 \varphi_{j} .
$$

The average $\langle\cos \varphi\rangle_{M F}$ in Eq. (3.25) should be calculated with the action $S_{\text {eff }}[\varphi]$ that can be obtained from $S[\varphi]$, Eq. (3.24) by making the replacement, Eq. (3.25), in $S_{J}[\varphi]$, Eq. (3.20). Actually, this average is the order parameter for the macroscopic superconductivity.

Assuming that the transition between the superconductor and insulator is of the second order we can find the critical point from the condition that at this point the mean field $\langle\cos 2 \varphi\rangle_{M F}$ vanishes. The corresponding equation determining the boundary of the superconducting state takes the form

$$
1=\frac{z J}{2} \int_{0}^{\beta} \Pi_{s}(\tau) d \tau
$$

where $z$ is the coordination number of the lattice and $\Pi_{s}(\tau)$ is determined by Eq. (3.6) assuming however that the average $\langle\ldots\rangle$ in that equation should be calculated with the action $S_{0}[\varphi]$, Eqs. (3.22) and (3.23).

In order to simplify calculations further we will neglect the off-diagonal terms in the matrix $E_{i j}$, Eq. (3.22), keeping the value $E_{0}(g)=\tilde{E}_{c}^{i i}$ only. This value can be written as

$$
E_{0}(g)=a^{d} \int \tilde{E}_{c}(\mathbf{q}) \frac{d^{d} \mathbf{q}}{(2 \pi)^{d}}
$$

where $\tilde{E}_{c}(\mathbf{q})$ is determined by Eq. 3.23). Then for $\Pi_{s}(\tau)$ instead of Eq. (3.7) we obtain the following expression

$$
\Pi_{s}(\tau)=\exp \left(-4 E_{0} \tau\right) .
$$

Solving Eq. (3.26) at $T \rightarrow 0$ one obtains the relation between the Josephson and Coulomb energies at the phase transition

$$
z J=8 E_{0}(g)
$$

Equation (3.29) agrees at small $g \ll 1$ (up to the coefficient) with the Anderson-Abeles criterion (Abeles, 1977). In this limit we obtain using Eq. (3.8) the critical value for the conductance in the form

$$
g_{c}^{*}=16 E_{0}(0) / \pi z \Delta .
$$

Samples with the tunneling conductances $g<g_{c}^{*}$ are insulators, while those with $g>g_{c}^{*}$ are superconductors. We note that this result suggests that even samples with weakly coupled granules $g_{c}^{*}<g \ll 1$ are still superconductors in spite of the fact that the corresponding array of normal grains with the same Coulomb interaction would be an insulator in this regime.

It is clear from Eq. (3.26) that the zero temperature result (3.30) holds also at finite temperatures so long as $T \ll E_{c}$. In the opposite limit $T \gg E_{c}$ (but still $T \ll$ $\left.T_{c 0}\right)$, one obtains $\Pi_{s}(\tau)=1$, which leads to the critical temperature

$$
T_{c}=\frac{1}{2} z J=\frac{1}{4} z \pi g \Delta .
$$

The results (3.30) and (3.31) correspond to the low coupling part (small $g$ ) of the phase diagram shown in Fig. (17).

As we have mentioned previously, the Anderson-Abeles criterion fails to produce the correct phase boundary between the insulating and superconducting states for arrays with a stronger coupling $g \geq 1$. According to the experiments (Jaeger et al., 1989; Orr et al., 1986) the superconductor-insulator transition is controlled by the resistance of the array only and the critical resistance $R_{c}$ (in the case of granular films) is close to the resistance quantum $R_{0} \approx 6.4 k \Omega$. The Coulomb interaction drops out from the result.

This interesting observation can be understood using Eqs. (3.29, 3.27 3.23 3.8). It is important to notice that the energy $E_{0}(g)$ depends on the dimensionality. Using Eqs. 3.23 3.27 we see that in $3 d$ arrays of the grains the function $E_{0}(g)$ equals

$$
E_{0}(g)=c \Delta / g
$$

where $c$ is a number of the order unity, while in $2 d$ we obtain with the logarithmic accuracy

$$
E_{0}(g)=\frac{2 \Delta}{3 \pi^{2} g} \ln \left(g E_{c} / \Delta\right)
$$

One can see from Eqs. (3.32) and (3.33) that the effective charging energy is independent on the originally strong Coulomb interaction in $3 d$ case and is almost independent on it in the $2 d$ case. Substituting Eqs. (3.32) and (3.33) into Eqs. (3.29), we come to the conclusion that in the limit $E_{C} \gg \Delta$ the superconductor-insulator transition occurs at $g_{c} \sim 1$, which is in agreement the with experiments.

Considerations presented in the original work (Chakravarty et al., 1987) lead to results qualitatively similar to those presented here. In particular, the 


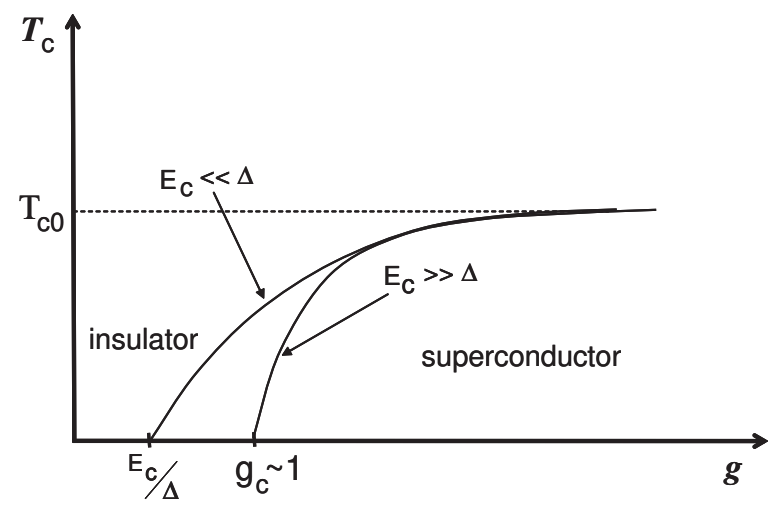

FIG. 17 The sketch of the phase diagram of a granular superconductor in coordinates critical temperature vs. tunnelling conductance for two cases $E_{c} \ll \Delta_{0}$ and $E_{c} \gg \Delta_{0}$. At large tunnelling conductances $g \gg 1$ the Coulomb interaction is screened due to electron intergrain tunnelling and the transition temperature is approximately given by the single grain BCS value. In the opposite case $g \ll 1$ the boundary between the insulating and superconducting states is obtained comparing the Josephson $E_{J}=g \Delta$ and Coulomb $E_{c}$ energies.

phase boundary at $T \rightarrow 0$ in two dimensional case was found to be

$$
\alpha_{c}^{2}=\frac{4}{3 \pi} \ln \left(1+\frac{3 \pi}{16} \alpha_{c} \frac{E_{c}}{\Delta}\right),
$$

where $\alpha_{c}$ is the critical value of the dimensionless conductance related to our conductance $g$ as $\alpha=\pi g$. The phase boundary following from the calculations of Chakravarty et al. (1987) is represented in Fig. 15.

Summarizing our discussion we present in Fig. 17 the phase diagram for a granular superconductor in the temperature vs. conductance coordinates for two cases of $E_{c} \ll \Delta$ and $E_{c} \gg \Delta$.

The strong coupling regime, $g>1$, can also be analyzed using the diagrammatic technique. This approach is advantageous in many respects since it allows one to find the corrections to the critical temperature in a straightforward and rigorous way not relying on the mean field approximation used in the treatment of the effective functional. This approach is considered in detail in Sec. III.D

We note that while the mean field theory gives qualitatively correct results, it certainly cannot be viewed as a complete description of the model, Eq. (3.17) because fluctuations near the mean field solutions can be important. In particular, Eq. (3.26) assumes that the system is not affected by the Josephson couplings so long as the mean value of $\varphi$ is zero. However, the Josephson coupling is known to be important even in the insulating state. In the simplest case of the insulating state with the low Josephson coupling the effect of the later can be included via the perturbation theory. As a result, the charging energy is reduced, $E_{c} \rightarrow \tilde{E}_{c}=E_{c}+\delta E_{c}$ with the correction $\delta E_{c}$ given by (Sachdev, 2001)

$$
\delta E_{c}=-z J / 2 .
$$

Closer to the superconductor - insulator transition the charging energy gets renormalized even more strongly and in the vicinity of the transition it is expected to have the scaling form, (Sachdev, 2001)

$$
E_{0}(g) \sim\left|g_{c}^{*}-g\right|^{\gamma},
$$

where $\gamma>0$ is the critical index. One may interpret the energy $E_{0}(g)$ as the increase of charging energy when adding a Cooper pair to a neutral grain. Exactly at the phase transition this energy vanishes according to Eq. (3.36) and this ensures the continuity of the Coulomb gap at the phase transition, since in the superconducting state this gap has to be zero.

The Coulomb gap is an important characteristic of the insulating state. In particular, it controls the low temperature conductivity that has an activation form

$$
\sigma \sim e^{-4 E_{0}(g) / T} .
$$

Experimentally measured conductivity of granular samples is, however, usually more complicated than that given by Eq. (3.37) and typically has a variable-range hopping type of behavior. Such a behavior can be obtained taking into account the electrostatic disorder neglected in the model, Eq. (3.17).

\section{Upper critical field of a granular superconductor}

As we already mentioned, the granular superconductors have rather unusual magnetic properties. In particular, at low temperatures, depending on the tunneling conductance $g$, the critical magnetic field is determined by either bulk formula (3.12) at $g>g^{*}$ or by the critical magnetic field of a single grain (3.11) at $g<g^{*}$, where the characteristic conductance $g^{*}$ is defined by Eq. B.9.9 ). In this section we will discuss in detail the dependence of the upper critical field $H_{c_{2}}$ of granular superconductors on the tunneling conductance $g$ at arbitrary temperatures. We will consider granular samples that are relatively good metals in their normal state, such that $g \gg 1$. This will allow us to neglect the effect of the suppression of the critical temperature by the Coulomb interaction and fluctuations.

\section{Critical field of a single grain}

We begin with a discussion of the critical magnetic field of a single grain. This problem was first considered long ago by Larkin (1965) by means of averaging the Gor'kov equations over disorder. Another way to consider this problem is based on using a later technique, namely, a semiclassical Usadel equation, (Usadel, 1970). This way is more convenient for our purposes since it can easily be generalized to the case of a granular array. Assuming that the transition between the superconducting and normal states is of the second order we define 
the critical field as a field at which the self-consistency equation (Abrikosov et al., 1965)

$$
\Delta(\mathbf{r})=\lambda_{0} \pi T \sum_{\omega} f_{\omega}(\mathbf{r}),
$$

where $\omega=\pi T(2 n+1)$, acquires a nontrivial solution under decrease of the magnetic field. The function $f_{\omega}(\mathbf{r})$ is a quasiclassical Green function that can be obtained from the anomalous Gor'kov Green function $F$ by integration over $\xi$, Eqs. (3.2) and (3.3). The Green function $f_{\omega}(\mathbf{r})$ can be determined from the Usadel equation that can be linearized close to the phase transition, see e.g. Kopnin, 2001)

$$
\left(|\omega|+D(-i \nabla-2 e \mathbf{A} / c)^{2} / 2\right) f_{\omega}(\mathbf{r})=\Delta(\mathbf{r}),
$$

where $D$ is the classical diffusion coefficient in the superconducting grain and $\mathbf{A}$ is the vector potential. We choose the vector potential corresponding to a homogeneous magnetic field $\mathbf{H}$ in the form $\mathbf{A}(\mathbf{r})=[\mathbf{H} \times \mathbf{r}] / 2$, which allows us to reduce Eq. (3.39) to the form

$$
\left(|\omega|-D \nabla^{2} / 2+2 D(e \mathbf{A} / c)^{2}\right) f_{\omega}(\mathbf{r})=\Delta(\mathbf{r}) .
$$

Equation (3.40) can be solved via perturbative expansion in $\mathbf{A}^{2}$. In the limit determined by Eq. (3.16), one can neglect dependence of the functions $f_{\omega}(\mathbf{r})$ and $\Delta(\mathbf{r})$ on coordinates. In the main approximation we can simply replace the term with $\mathbf{A}^{2}(\mathbf{r})$ in Eq. (3.40) by its average over the volume of the grain. Thus, we obtain the simple algebraic equation relating constant components of $f$ and $\Delta$

$$
f_{\omega}(|\omega|+\alpha)=\Delta
$$

where the depairing parameter $\alpha$ is the case of a spherical grain with the radius $R$ is

$$
\alpha=R^{2} D(e H / c)^{2} / 5 .
$$

Substituting the solution of Eq. (3.41) into Eq. (3.38) we come to the standard equation (Abrikosov and Gor'kov, 1961) relating the critical temperature $T_{c}$ to the depairing parameter $\alpha$, Eq. (3.42),

$$
\ln \left(T_{c} / T_{c 0}\right)=\psi(1 / 2)-\psi\left(1 / 2+\alpha / 2 \pi T_{c}\right) .
$$

Here $\psi(x)$ is the digamma function and $T_{c 0} \equiv T_{c}(H=0)$ is the single grain superconducting critical temperature in the absence of the magnetic field. One can see that at zero temperature the superconductivity is destroyed at

$$
\alpha_{c 0}=\Delta_{0} / 2
$$

where $\Delta_{0}$ is the superconducting order parameter at $T, H=0$ that is related in the standard way to the critical temperature $T_{c 0}$ in the absence of the magnetic field as $\Delta_{0}=\pi T_{c 0} / \gamma$ with $\ln \gamma \approx 0.577$ being the Euler constant. For a single grain of a spherical shape we obtain Eq. (3.11) for the critical field $H_{c}^{\mathrm{gr}}$ at zero temperature.

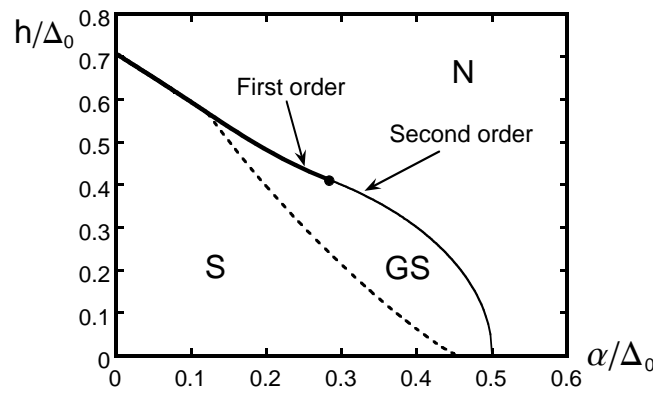

FIG. 18 Phase diagram of a superconducting grain at $T=0$ in coordinates Zeeman energy $h$ vs. pairbreaking parameter $\alpha$. The dashed line separates the gapless (GS) and gapful (S) superconducting states. First and second order phase transition lines are shown by thick and thin solid lines, respectively (Beloborodov et al., 2006).

The above approach can be easily generalized to take into account the Zeeman splitting by making the shift $|\omega| \rightarrow|\omega|+h \operatorname{sign} \omega$, where $h$ is the Zeeman energy $h=$ $\mu_{B} H$. Then, Eq. (3.43) is modified to

$$
\ln \left(T_{c} / T_{c 0}\right)=\psi(1 / 2)-\operatorname{Re}\left[\psi\left(1 / 2+(\alpha+i h) / 2 \pi T_{c}\right)\right] .
$$

We note that Eq. (3.45) is valid as long as the phase transition is of the second order. However, in the limit where the Zeeman effect dominates the orbital one Clogston (1960) the phase transition is known to be first order. Analysis of the first order phase transition at finite temperatures is rather involved and here we present only the corresponding phase diagram at zero temperature (Fig. 18) that illustrates how the second order phase transition turns into the first order one (Beloborodov et al., 2006). To simplify the discussion we assume that the Clogston limit is not reached and Eq. (3.45) describing the second order phase transition is applicable.

At zero temperature, Eq. (3.45) reduces to (Beloborodov et al., 2000)

$$
\alpha^{2}+h^{2}=\Delta_{0}^{2} / 4,
$$

such that Eq. (3.11) for the critical field $H_{c}^{g r}$ in the presence of the Zeeman coupling can simply be generalized via the substitution

$$
\Delta_{0} \rightarrow \tilde{\Delta}_{0} \equiv \sqrt{\Delta_{0}^{2}-(2 h)^{2}} .
$$

\section{Critical magnetic field of a granular sample}

According to subsection III.C.1 the effect of the magnetic field on a single grain reduces to the renormalization of the Matsubara frequency

$$
\omega \rightarrow \tilde{\omega}=\omega+i h+\alpha .
$$

So, considering the critical temperature of the whole granular sample one can take into account the local single grain effects via the frequency renormalization, (3.48). 
The critical temperature of the granular array can be again found from the linearized Usadel equation that can be written for the granular sample in the Fourier representation as

$$
(|\omega|+(g \delta / 2) \lambda(\mathbf{q}-2 e \mathbf{A} / c)) f(\mathbf{q})=\Delta(\mathbf{q})
$$

where $\lambda(\mathbf{q}-2 e \mathbf{A} / c)=2 \sum_{\mathbf{a}}(1-\cos [(\mathbf{q}-2 e \mathbf{A} / c) \mathbf{a}])$ and $\mathbf{q}$ is the quasimomentum of the periodic lattice. In the regime under consideration, Eq. (3.16), the magnetic flux per a unite cell of the lattice is always small. This allows us to find the lowest eigenvalue of the operator $\lambda(\mathbf{q}-$ $2 e \mathbf{A} / c)$ as the lowest Landau level of the operator for the corresponding continuous system $a^{2}(\hat{\mathbf{q}}-2 e \mathbf{A} / c)^{2}$, which gives $2 e \mathrm{Ha}^{2} / \mathrm{c}$.

Thus, the evaluation of the orbital effect that originates from flux accumulation on scales involving many grains is very similar to the analogous effect in the homogeneously disordered samples, which is a direct consequence of the smallness of the magnetic flux per unite cell. Now one can see that the orbital effect that comes from large distances simply gives an additional contribution $g \delta a^{2} e H / c$ to the depairing parameter $\alpha$. Using Eq. (2.3) for the effective diffusive coefficient $D_{\text {eff }}$ we obtain for the total depairing parameter including both single grain and bulk orbital effects of the magnetic field

$$
\alpha=R^{2} D(e H / c)^{2} / 5+D_{e f f} e H / c .
$$

We note that although Eq. (3.50) has been obtained within the periodic cubic lattice array model it is in fact more general. Indeed, the first term in the right hand side of Eq. (3.50) represents the single grain effect and is sensitive to the grain size only. The second term, being expressed in terms of the effective diffusive coefficient $D_{\text {eff }}$, is not sensitive to the structure of the array at all.

Comparing the two contributions to the depairing parameter $\alpha$ in Eq. (3.50) we obtain immediately the value $H^{*}$ characterizing the crossover from the single grain to the bulk orbital effects

$$
H^{*}=\frac{5 \phi_{0}}{\pi R^{2}} \frac{D_{e f f}}{D}
$$

Equations (3.50) and (3.45) determine the superconducting transition line in the coordinates $T$ vs. $H$ plane.

In the limit of zero temperature $T \rightarrow 0$, Eq. 3.45 reduces to Eq. (3.46), which allows us to find a simple expression for the critical filed at $T=0$ resolving Eq. (3.50) with respect to $H$ :

$$
H_{c}(T=0)=\frac{5 \phi_{0}}{2 D \pi R^{2}}\left(\sqrt{D_{e f f}^{2}+\frac{2 \tilde{\Delta}_{0} R^{2} D}{5}}-D_{e f f}\right),
$$

where $\tilde{\Delta}_{0}$ defined by Eq. (3.47) takes into account the Zeeman effect.

\section{Suppression of the superconducting critical temperature}

The BCS theory gives a very accurate description of superconductors in bulk well conducting metals. However, in strongly disordered or granular metals there can be considerable deviations from this theory. This concerns, in particular, the superconducting transition temperature.

In this subsection we consider suppression of the superconductivity in granular metals at large tunneling conductances between the grains, $g \gg 1$. In this limit, all properties of the system should be close to those following from the BCS theory. The mean field transition temperature $T_{c}$ of the granular superconductors has to be close to that of the corresponding disordered bulk superconductor. A difference between the granular and disordered bulk superconductors may appear only in corrections to the BCS theory. These corrections will be discussed in the present subsection.

The main mechanisms of the suppression of the superconductivity are the Coulomb repulsion and fluctuations of the superconducting order parameter. For example, disorder shifts significantly the superconducting transition temperature in the $2 d$ thin films (Finkelstein, 1987, 1994; Ishida and Ikeda, 1998; Larkin, 1999; Maekawa et al., 1983; Maekawa and Fukuvama, 1982; Ovchinnikov, 1973). The physical reason for the suppression of the critical temperature is that in thin films the interaction amplitude in the superconducting channel decreases due to peculiar disorder-induced interference effects that enhance the effective Coulomb interaction. We refer to this mechanism of the superconductivity suppression as to the fermionic mechanism.

The superconducting transition temperature can also be reduced by the fluctuations of the order parameter, the effect being especially strong in low dimensions. The corresponding mechanism of the superconductivity suppression is called the bosonic mechanism. In particular, the bosonic mechanism can lead to the appearance of the insulating state at zero temperature (Efetov, 1980; Fisher, 1990; Simanek, 1994).

In this subsection we discuss the suppression of the superconductivity in terms of the perturbation theory. Although this method is restrictive and cannot be used to study the superconductor -insulator transition, it is useful in a sense that both the relevant mechanisms of the reduction of the critical temperature can be included systematically within the same framework. The usefulness of the perturbative calculations has been demonstrated in the study of properties of normal granular metals in Sec. II.D where the new important energy scale $\Gamma$, Eq. (2.2) was introduced. In view of these findings one may expect that the correction to the superconducting transition temperature can be different depending on whether the temperature is larger or smaller than the energy scale $\Gamma$.

We start with the following Hamiltonian $\hat{H}_{s}$ describing 
a)

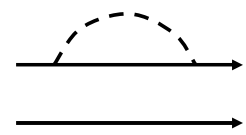

c)

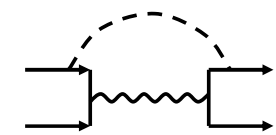

b)

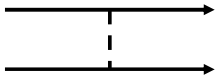

d)

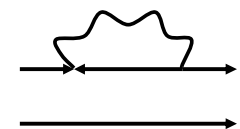

FIG. 19 Diagrams a) - c) describe the correction to the superconducting transition temperature due to Coulomb repulsion. The diagram d) describes correction to the transition temperature due to superconducting fluctuations. All diagrams are shown before averaging over the impurities. The solid lines denote the electron propagators, the dashed lines describe screened Coulomb interaction and the wavy lines describe the propagator of superconducting fluctuations (Beloborodov et al., 2005a).

the granular system

$$
\hat{H}_{s}=\hat{H}+\hat{H}_{e-p h},
$$

where the Hamiltonian $\hat{H}$ for the normal granular metals is given by Eq. (2.15) and $\hat{H}_{e-p h}$ is an additional electronphonon interaction on each grain

$$
\hat{H}_{e-p h}=-\lambda \sum_{i, k, k^{\prime}} a_{i, k}^{+} a_{i,-k}^{+} a_{i,-k^{\prime}} a_{i, k^{\prime}},
$$

where $i$ labels the grains, $k \equiv(\mathbf{k}, \uparrow),-k \equiv(-\mathbf{k}, \downarrow) ; \lambda>$ 0 is the interaction constant; $a_{i, k}^{+}\left(a_{i, k}\right)$ are the creation (annihilation) operators for an electron in the state $k$ of the $i$-th grain.

The superconducting transition temperature can be found by considering the anomalous Green function $F$ in the presence of an infinitesimal source of pairs $\Delta$, (Ovchinnikov, 1973). Neglecting fluctuations and interaction effects the anomalous Green function $F$ is given by the expression (Abrikosov et al., 1965)

$$
F(\xi, \omega)=\Delta /\left(\omega^{2}+\xi^{2}\right),
$$

where $\xi=\mathbf{p}^{2} / 2 m-\mu$, and $\omega=2 \pi T(n+1 / 2)$ is the fermionic Matsubara frequency. The suppression of the transition temperature $T_{c}$ is determined by the correction to the function $F(\xi, \omega)$

$$
\frac{\Delta T_{c}}{T_{c}}=\frac{T}{\Delta} \int d \xi \sum_{\omega} \delta F(\xi, \omega),
$$

where the function $\delta F(\xi, \omega)$ represents the leading order corrections to the anomalous Green function $F(\xi, \omega)$ due to fluctuations of the order parameter and Coulomb interaction. All diagrams (before impurity averaging) contributing to the suppression of the transition temperature in Eq. (3.56) are shown in Fig. 20] One can see

that there exist two qualitatively different classes of diagrams. First, the diagrams a) - c) describe corrections to the transition temperature due to Coulomb repulsion and correspond to the so called fermionic mechanism of the suppression of superconductivity. The second type, diagram d), describes a correction to the transition temperature due to the superconducting fluctuations and represents the bosonic mechanism. The details of calculations are presented in Ref. (Beloborodov et al., 2005a). They are rather straightforward and therefore we present and discuss below only the final results for the suppression of $T_{c}$.

It is convenient to separate corrections due to the bosonic and fermionic mechanisms from each other and write the result for the suppression $\Delta T_{c}$ of the superconductor transition temperature as

$$
\frac{\Delta T_{c}}{T_{c}}=\left(\frac{\Delta T_{c}}{T_{c}}\right)_{b}+\left(\frac{\Delta T_{c}}{T_{c}}\right)_{f},
$$

where the two terms in the right hand side correspond to the bosonic and fermionic mechanisms, respectively. The critical temperature $T_{c}$ in Eq. (3.57) is the BCS critical temperature.

It is shown by Beloborodov et al. (2005a) that at high transition temperatures, $T_{c}>\Gamma$, the fermionic correction to the superconducting transition temperature does not depend on the dimensionality of the sample and takes the form

$$
\left(\frac{\Delta T_{c}}{T_{c}}\right)_{f}=-c_{1} \frac{\delta}{T_{c}}, \quad d=2,3 .
$$

where $c_{1}=7 \zeta(3) / 2 \pi^{2}-(\ln 2) / 4$ is the numerical coefficient and $d$ is the dimensionality of the array of the grains.

In contrast, in the low temperature regime, $T_{c}<\Gamma$, the fermionic mechanism correction to the superconducting transition temperature depends on the dimensionality of the sample and is given by (Beloborodov et al., 2005a)

$$
\left(\frac{\Delta T_{c}}{T_{c}}\right)_{f}=-\left\{\begin{array}{ll}
\frac{A}{2 \pi g} \ln ^{2} \frac{\Gamma}{T_{c}}, & d=3 \\
\frac{1}{24 \pi^{2} g} \ln ^{3} \frac{\Gamma}{T_{c}}, & d=2
\end{array},\right.
$$

where $A=a^{3} \int d^{3} q /(2 \pi)^{3} \lambda_{\mathbf{q}}^{-1} \approx 0.253$ is the dimensionless constant, $a$ is the size of the grain and $\lambda_{\mathbf{q}}$ is given by Eq. (2.30). Note that in the low temperature regime, $T_{c}<\Gamma$, the correction to the critical temperature in two dimensions agrees with the one obtained for homogeneously disordered superconducting films provided the substitution $\Gamma \rightarrow \tau^{-1}$ is done.

The correction to the transition temperature due to the bosonic mechanism in Eq. (3.57) is qualitatively different from the fermionic one. It remains the same in both the regimes $T<\Gamma$ and $T>\Gamma$ and is given by (Beloborodov et al., 2005a)

$$
\left(\frac{\Delta T_{c}}{T_{c}}\right)_{b}=-\left\{\begin{array}{lc}
\frac{14 A \zeta(3)}{\pi^{3}} \frac{1}{g}, & d=3 \\
\frac{7 \zeta(3)}{2 \pi^{4} g} \ln \frac{g^{2} \delta}{T_{c}}, & d=2
\end{array},\right.
$$


where $\zeta(x)$ is the zeta-function and the dimensionless constant $A$ is written below Eq. (3.59).

Note that the energy scale $\Gamma$ does not appear in this bosonic part of the suppression of superconducting temperature in Eq. (3.60). This stems from the fact that the characteristic length scale for the bosonic mechanism is the coherence length $\xi_{\text {eff }}$ that is assumed to be much larger than the size of the single grain. The result for the two dimensional case in Eq. (3.60) is written with a logarithmic accuracy assuming that $\ln \left(g^{2} \delta / T_{c}\right) \gg 1$.

We see from Eqs. (3.59) and (3.60) that the suppression of the superconducting transition temperature becomes stronger with diminishing the coupling $g$ between the grains. Of course, the calculations may be justified only when the correction to the transition temperature is much smaller than the temperature itself.

The above expression for the correction to the transition temperature due to the bosonic mechanism was obtained in the lowest order in the propagator of superconducting fluctuations and holds therefore as long as the value for the critical temperature shift given by Eq. (3.60) is larger than the Ginzburg region $(\Delta T)_{G}$

$$
(\Delta T)_{G} \sim \begin{cases}\frac{1}{g^{2}} \frac{T_{c}^{2}}{g \delta} & d=3, \\ \frac{T_{c}}{g} & d=2 .\end{cases}
$$

Comparing the correction to the transition temperature $T_{c}$ given by Eq. (3.60) with the width of the Ginzburg region, Eq. (3.61), one concludes that for $3 d$ granular metals the perturbative result (3.60) holds if

$$
T_{c} \ll g^{2} \delta .
$$

In two dimensions the correction to the transition temperature in Eq. (3.60) is only logarithmically larger than $(\Delta T)_{G}$ in Eq. (3.61). The two dimensional result (3.60) holds with the logarithmic accuracy in the same temperature interval (3.62) as for the three dimensional samples.

Note that inside the Ginzburg region higher order fluctuation corrections become important. Moreover, the non perturbative contributions that appear, in particular, due to superconducting vortices should be taken into account as well. These effects destroy the superconducting long range order and lead to Berezinskii-KosterlitzThouless transition in $2 d$ systems. The suppression of the transition temperature in the limit $T>g^{2} \delta$ in $3 d$ should be studied considering strong critical fluctuations within the Ginzburg-Landau free energy functional.

It follows from Eq. (3.58) that in the limit $T \gg \Gamma$ the fermionic mechanism of the suppression of the superconductivity is no longer efficient. This can be seen rather easily in another way using the phase approach presented in Sec. II.B.4 After the decoupling of Coulomb term (2.39) by the integration over the auxiliary field $\bar{V}$ and gauge transformation (2.42) the phase enters the tunneling term, Eq. (2.53) only. However, this term is not important in the limit $T \gg \Gamma$ and we conclude that the long range part of the Coulomb interaction leading to charging of the grains is completely removed in this limit. Therefore, the effect of the Coulomb interaction on the superconducting transition temperature must be small and this is seen from Eq. (3.58). This conclusion matches well the fact that the upper limit in the logarithms in Eq. 3.59) is just $\Gamma$ and, at temperatures exceeding this energy, the logarithms should disappear.

At lower temperatures $T<\Gamma$ the phase description is not applicable and, as a consequence, we obtain the non-trivial result, Eq. (3.59). This result is of the pure quantum origin, and the interference effects are very important for its derivation (one should consider a contribution of the diffusion modes). In the high temperature limit $T \gg \Gamma$ the interference effects are suppressed and this is the reason why the fermionic mechanism of the suppression of the superconductivity is no longer efficient.

The results obtained, Eqs. (3.58) - (3.62), suggest an experimental method of extracting the information about the morphology of the samples from the $T_{c}$ data. Indeed, one can study the dependence of the superconducting transition temperature $T_{c}$ of the granular metals as a function of the tunneling conductance $g$ by comparing several granular samples with different tunneling conductances (different oxidation coating). The experimental curves for the suppression of $T_{c}$ should have a different slope at high $T_{c}>\Gamma$ and low $T_{c}<\Gamma$ critical temperatures due to the fact that the suppression of the superconductivity is given by the two different mechanisms. The information on the morphology of the sample, i.e. whether the samples are homogeneously disordered or granular is then obtained from the dependencies of the critical temperatures on the tunneling conductance $T_{c}(g)$. (We remind the reader that the scale $\Gamma$ exists in the granular samples only).

Another consequence of Eqs. (3.58) - (3.62) is the following: since at low critical temperatures $T_{c} \ll \Gamma$ the suppression of the superconductivity in granular metals is provided by the fermionic mechanism and coincides, upon the substitution $\Gamma \rightarrow \tau^{-1}$, with the proper result for homogeneously disordered samples, Ovchinnikov, 1973), one can generalize the renormalization group result by Finkelstein (1987) for the $T_{c}$ suppression. The latter result obtained for homogeneously disordered films can be directly applied to the granular superconductors by making the proper substitution for the diffusion coefficient $D=\Gamma a^{2}$, where $a$ is the size of the single grain.

\section{E. Magnetoresistance of granular superconductors}

In this subsection we discuss magnetoresistance of a granular superconductor in a strong magnetic field. We start our consideration with a discussion of the experiment (Gerber et al., 1997) where transport properties a system of $A l$ superconducting grains in a strong magnetic field were studied. The samples were three dimensional and quite homogeneous with a typical grain diameter $120 \pm 20 \AA$. Bulk superconductivity could be destroyed by application the strong magnetic field leading to the 
appearance of a finite resistivity. Magnetic field above $17 T$ was enough to destroy even the superconducting gap within each grain.

The dependence of the resistivity on the magnetic field observed in Gerber et al. (1997) was not simple. Although at extremely strong fields the resistivity was almost independent of the field, it increased when decreasing the magnetic field. Only at sufficiently weak magnetic fields the resistivity started to decrease and finally the samples displayed superconducting properties. A similar behavior had been reported in a number of publications (Gantmakher et al., 1996; Parthasarathv et al., 2004).

A negative magnetoresistance due to weak localization effects is not unusual in disordered metals Altshuler et al., 1980; Lee and Ramakrishnan, 1985). However, the characteristic magnetic field in experiment (Gerber et al., 1997) was several tesla such that all weak localization effects had to be strongly suppressed.

We want to present an explanation for this unusual behavior based on consideration of superconducting fluctuations. At first glance this idea looks counterintuitive, because, naively, superconducting fluctuations are expected to increase the conductivity. Nevertheless, it turns out that the magnetoresistance of a good granulated metal $(g \gg 1)$ in a strong magnetic field, $H>H_{c}$, and at low temperature, $T \ll T_{c}$, is negative. In our model, the superconducting gap in each granule is assumed to be suppressed by the strong magnetic field. All the interesting behavior considered below originates from the superconducting fluctuations that lead to a suppression of the density of states (DOS) and decrease the conductivity.

Theory of superconducting fluctuations near the transition into the superconducting state has been developed long ago (Abrahams et al., 1970; Aslamazov and Larkin, 1968; Maki, 1968a, b; Thompson, 1970) (for a review see Larkin and Varlamov (2005)). Above the transition temperature $T_{c}$, non-equilibrium Cooper pairs are formed and a new channel of charge transfer opens (AslamazovLarkin contribution) (Aslamazov and Larkin, 1968). This correction always gives positive contribution to the conductivity. Another fluctuation contribution comes from a coherent scattering of the electrons forming a Cooper pair on impurities (Maki-Thompson contribution) (Maki, 1968a b; Thompson, 1970). This correction also results in a positive contribution to conductivity, though, in principle, the sign of it is not prescribed.

Formation of the non-equilibrium Cooper pairs results also in a fluctuational gap in the one-electron spectrum (Abrahams et al., 1970) but in conventional (non granular) superconductors the first two mechanisms are more important near $T_{c}$ and the conductivity increases when approaching the transition.

The total conductivity for a bulk sample above the transition temperature $T_{c}$ can be written in the following form

$$
\sigma=\sigma_{\text {Drude }}+\delta \sigma,
$$

where $\sigma_{\text {Drude }}=\left(e^{2} \tau n\right) / m$ is the conductivity of a normal metal without electron-electron interaction, $\tau$ is the elastic mean free time, $m$ and $n$ are the effective mass and the density of electrons, respectively. In Eq. (3.63), $\delta \sigma$ is a correction to the conductivity due to the Cooper pair fluctuations

$$
\delta \sigma=\delta \sigma_{D O S}+\delta \sigma_{A L}+\delta \sigma_{M T}
$$

where $\delta \sigma_{D O S}$ is the correction to the conductivity due to the reduction of the DOS and $\delta \sigma_{A L}$ and $\delta \sigma_{M T}$ stand for the Aslamazov-Larkin (AL) and Maki-Thompson (MT) contributions to the conductivity. Close to the critical temperature $T_{c}$ the $\mathrm{AL}$ correction is more important than both the MT and DOS corrections and its contribution can be written as follows (Aslamazov and Larkin, 1968)

$$
\frac{\delta \sigma_{A L}}{\sigma_{\text {Drude }}}=\gamma\left(\frac{T_{c}}{T-T_{c}}\right)^{\beta},
$$

where $\gamma$ is a small dimensionless positive parameter, $\gamma \ll 1$, depending on dimensionality and $\beta=1 / 2$ for the three-dimensional case $(3 d), 1$ for $2 d$ and $3 / 2$ for quasi$1 d$. Equation (3.65) is valid for $\delta \sigma_{A L} / \sigma_{\text {Drude }} \ll 1$.

For quite a long time the superconducting fluctuations have been studied near the critical temperature $T_{c}$ in a zero or a weak magnetic field. Only recently the limit of very low temperature, $T \ll T_{c}$, and a strong magnetic field, $H>H_{c}$ was considered (Beloborodov and Efetov, 1999; Beloborodov et al., 2000) and some qualitatively new effects were predicted. In this subsection we concentrate on this limit.

As in conventional bulk superconductors, we can write corrections to the classical conductivity $\sigma_{0}$, Eq. (2.1), as a sum of corrections to the DOS, Aslamazov-Larkin (AL), $\sigma_{A L}$, and Maki-Thompson (MT), $\sigma_{M T}$, corrections. Diagrams describing these contributions are represented in Fig. 20. They have the conventional form but the wavy lines standing for the superconducting fluctuations should be written taking into account the granular structure. It turns out that in the regime $T \ll T_{c}$ and $H>H_{c}$ the DOS corrections plays a very important role: This correction reaches its maximum at $H \rightarrow H_{c}$, where $H_{c}$ is the field destroying the superconducting gap in the single grain. At zero temperature and close to the critical field $H_{c}$ such that $\Gamma / \Delta_{0} \gg h$, where $h=\left(H-H_{c}\right) / H_{c}$, the maximum value $\delta \sigma_{D O S}$ for $3 d$ granular superconductors is (Beloborodov et al., 2000)

$$
\frac{\delta \sigma_{D O S}}{\sigma_{0}}=-\frac{1}{3 g} \frac{\Gamma}{\Delta_{0}} \ln \left(\frac{\Delta_{0}}{\Gamma}\right) .
$$

We see from Eq. (3.66) that the correction to the conductivity $\delta \sigma_{D O S}$ i) is negative and its absolute value decreases when the magnetic field increases, ii) it is smaller 
a)

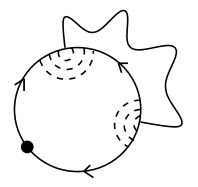

b)

c)

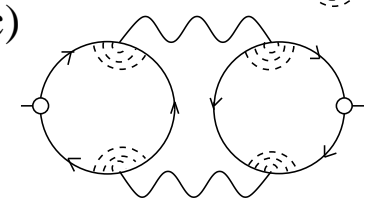

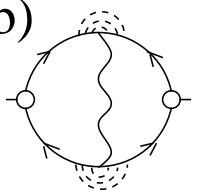

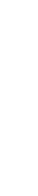

FIG. 20 Diagram a) describes correction to DOS, diagrams b) and c) describe corrections to conductivity due to superconducting fluctuations. The wavy lines denote the propagator of the fluctuations, the dashed lines stand for the impurity scattering (Beloborodov and Efetov, 1999).

than unity and it becomes comparable with $\sigma_{0}$ when the tunneling conductance $g \sim 1$. However, such values of $g$ mean that we would be in this case not far from the metal-insulator transition. Then, we would have to take into account all localization effects, which is not done here.

Even in the limit of strong magnetic fields, $H \gg H_{c}$, the correction to $\sigma_{0}$ can still be noticeable (Beloborodov et al., 2000)

$$
\frac{\delta \sigma_{D O S}}{\sigma_{0}}=-\frac{1}{3 g} \frac{\Gamma}{E_{0}(H)} \ln ^{-1}\left[\frac{E_{0}(H)}{\Delta_{0}}\right]
$$

where, for spherical grain, $E_{0}(H)=\frac{2}{5}\left(\frac{e H R}{c}\right)^{2} D$ with $D$ being the diffusion coefficient in the single grain. Equation (3.67) shows that in the region $H \gg H_{c}$ the correction to the conductivity behaves essentially as $\delta \sigma_{D O S} \sim$ $H^{-2}$, which is a rather slow decay.

We emphasize that the correction to the conductivity coming from the DOS, Eqs. (3.66) and (3.67) remains finite in the limit $T \rightarrow 0$, thus indicating the existence of the virtual Cooper pairs even at $T=0$.

In order to calculate the entire conductivity we must investigate the AL and MT contributions (Figs. 20, and 20]). Near $T_{c}$ these contributions are most important leading to an increase of the conductivity. At low temperatures $T \ll T_{c}$ and strong magnetic fields $H>H_{c}$ the situation is completely different. It turns out that both the AL and MT contributions vanish in the limit $T \rightarrow 0$ at all $H>H_{c}$ and thus, the correction to the conductivity comes from the DOS only.

So, at low temperatures, estimating the total correction to the classical conductivity $\sigma_{0}$, Eq. (2.42), one may use the formulae (3.66) and (3.67). We present the final result for $\mathrm{AL}$ correction shown in Fig. 201 at $T \ll T_{c}$ and $h \ll \Gamma / \Delta_{0}$ for $3 d$ granular superconductors (Beloborodov et al., 2000)

$$
\frac{\delta \sigma_{A L}}{\sigma_{0}} \sim \frac{1}{g} \frac{T^{2}}{\Delta_{0}^{3 / 2} \Gamma^{1 / 2}}\left(\frac{H_{c}}{H-H_{c}}\right)^{3 / 2} .
$$

It follows from Eq. (3.68) that at low temperatures the AL correction to the conductivity is proportional to the square of the temperature $\delta \sigma_{A L} \sim T^{2}$ and vanishes in the limit $T \rightarrow 0$.

For MT corrections shown in Fig. 20] one obtains in the limit $T \ll T_{c}$ and $h \ll \Gamma / \Delta_{0}$ for $3 d$ granular superconductors (Beloborodov et al., 2000)

$$
\frac{\sigma_{M T}}{\sigma_{0}} \sim \frac{1}{g} \frac{\Gamma}{\Delta_{0}} \frac{T^{2}}{\Delta_{0}^{2}}
$$

The temperature and magnetic field dependence of $\delta \sigma_{A L}$ and $\delta \sigma_{M T}$ is rather complicated but they are definitely positive. The competition between these corrections and $\sigma_{D O S}$ determines the sign of the magnetoresistance. One can see from Eqs. (3.68) and (3.69) that both the AL and MT contributions are proportional at low temperatures to $T^{2}$. Therefore the $\sigma_{D O S}$ in this limit is larger and the magnetoresistance is negative for all $H_{c}$. In contrast, at $T \sim T_{c}$ and close to $H_{c}$, the AL and MT corrections can become larger than $\sigma_{D O S}$ resulting in a positive magnetoresistance in this region. Far from $H_{c}$ the magnetoresistance is negative again.

In the above considerations we did not take into account interaction between the magnetic field and spins of the electrons. This approximation is justified if the size of the grains is not very small. Then, the orbital magnetic critical field $H_{c}^{\text {or }}$ destroying the superconducting gap is smaller than the paramagnetic limit $\mu H_{c}^{Z} \approx \Delta_{0}$ and the orbital mechanism dominates the magnetic field effect on the superconductivity. However, the Zeeman splitting leading to the destruction of the superconducting pairs can become important if one further decreases the size of the grains. The results for conductivity corrections including Zeeman splitting can be found in Ref. (Beloborodov et al., 2000).

The results presented in this subsection show that, even if the superconducting gap in each granule is destroyed by the magnetic field, the virtual Cooper pairs can persist up to extremely strong magnetic fields. However, the contribution of the Cooper pairs to transport is proportional at low temperatures to $T^{2}$ and vanishes in the limit $T \rightarrow 0$. In contrast, they reduce the oneparticle density of states in the grains even at $T=0$, thus diminishing the macroscopic conductivity. The conductivity can reach its classical value only in extremely strong magnetic fields when all the virtual Cooper pairs do not exist anymore. This leads to the negative magnetoresistance.

Qualitatively, the results for resistivity behavior of granular superconductors at low temperatures $T \ll T_{c}$ and strong magnetic fields $H>H_{c}$ are summarized in Fig. 21 The resistivity $R$ at low temperatures grows monotonously when decreasing the magnetic field and it reaches asymptotically the value of the classical resistivity $R_{0}$ only at extremely strong magnetic fields. The transition into the superconducting state occurs at a field $H_{c_{2}}$ that may be lower than $H_{c}$ 


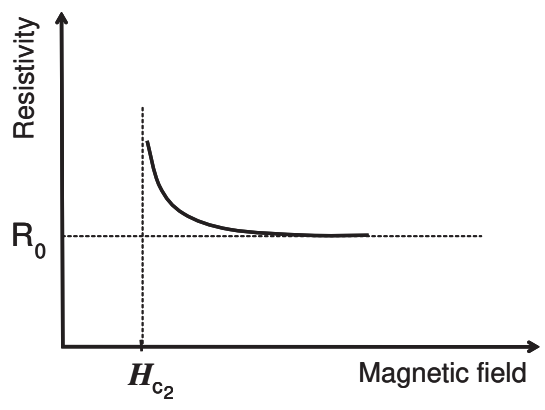

FIG. 21 Schematic picture for resistivity behavior of the granulated superconductors as a function of the magnetic field at low temperatures $T \ll T_{c}$. The resistivity at low temperatures grows monotonously when decreasing the magnetic field. It reaches asymptotically the value of the classical resistivity $R_{0}$ only at extremely strong magnetic fields. The transition into the superconducting state occurs at a field $H_{c_{2}}$ (Beloborodov et al., 2000).

\section{DISCUSSION OF THE RESULTS}

\section{A. Quantitative comparison with experiments}

The models of the normal and superconducting granular electronic systems considered in this review are relevant to many experiments on different materials and we have presented quite a few of them. This is not accidental because we selected for the presentation such theoretical results that could be relevant for existing experiments.

On the one hand, the review is not as complete as it could be because we did not include many interesting theoretical works where such physical quantities, as tunneling density of states (Beloborodov et al., 2004a), thermal conductivity (Beloborodov et al., 2005b; Biagini et al., 2005b; Loh and Tripathi, 2006), etc. were considered. The only reason for this omission is that at present we are not aware of clear measurements of these quantities in the granular materials.

On the other hand, this gave us the opportunity to concentrate on experimentally well studied phenomena. Some of them, like the logarithmic temperature dependence of the conductivity of well conducting granular metals or the hopping like temperature dependence of the insulating very regular granular systems remained unexplained for several decades. As we have seen, these dependencies and many interesting features of the experiments can be explained using rather simple models of the metallic grains. The simplicity of the models and a small number of parameters characterizing the system suggests that there should be not only a good qualitative but also a quantitative agreement between the theory and experiment.

So, we present now a quantitative comparison of several theoretical results with experimental data. Of course, we cannot discuss here all existing measurements and therefore only three different experiments are considered.

\section{Logarithmic temperature dependence of the conductivity}

In this subsection we will compare with experiments the logarithmic temperature dependence of the conductivity, Eq. (2.72). This formulae should be applicable at not very low temperature that simplifies the experimental check. Specially prepared granular metals like those considered in Refs. (Abeles et al., 1975; Gerber et al., 1997; Simon et al., 1987) should be the first objects of the application of the theory developed.

As an example, we compare now Eq. (2.72) with the experimental results of Gerber et al. (1997) on films made of $A l$ grains embedded in an amorphous $G e$ matrix. At low temperatures, superconductivity in $A l$ grains was destroyed by a strong magnetic field. Depending on the coupling between the grains (extracted from the conductivity at room temperatures) the samples of the experiment (Gerber et al., 1997) were macroscopically either in an insulating state with the temperature dependence of the resistivity $R \sim \exp \left(a / T^{1 / 2}\right)$ or in a "metallic" one. However, the resistivity of the metallic state depended on temperature and the authors suggested a power law, $R \sim T^{\alpha}$, with an exponent $\alpha \ll 1$. As the exponent $\alpha$ for the "metallic" sample was small we can argue that Eq. (2.72) should not be worse for fitting the experimental data. Then, we can estimate the exponent $\alpha$ without using fitting parameters.

The sample of the experiment (Gerber et al., 1997) had the room temperature resistivity $R_{0}=7.3 \times 10^{3} \Omega \mathrm{cm}$. The diameter of the grains was $120 \pm 20 \AA$, which allows, using the value $\hbar / e^{2}=4.1 \times 10^{-3} \Omega$, and the relation between the conductivity and conductance for $3 \mathrm{~d}$ samples $\sigma=2 e^{2} g / \hbar a$, to estimate the dimensionless tunneling conductance as $g=0.34$. Taking $d=3$ and $(z=6)$ in Eq. (2.72) we obtain $\alpha=0.15$, which agrees fairly well with the experimental value $\alpha=0.117$. Keeping in mind that the theoretical dependence (2.72) was obtained for a periodic cubic array while in realistic samples the coordination number can be only approximately close to 6 one can hardly hope for a better agreement.

A logarithmic dependence of the resistivity on temperature has been observed in other granular materials. In Ref. (Simon et al., 1987) a granular cermet consisting of $\mathrm{Nb}$ grains in a boron nitride insulating matrix was studied. Again, at small coupling between the grains the temperature dependence of the resistivity $\exp \left(a / T^{1 / 2}\right)$ was observed in a very broad interval of temperatures. The resistivity of samples with a strong coupling between the grains was very well described by the law

$$
R=R_{0} \ln \left(T_{0} / T\right),
$$

which is close to Eq. 2.72 provided the temperature interval is not very large, such that the variation of the resistivity is small. However, Eq. (4.1) gave a good description for the temperature dependence of the resistivity in a very broad region and the changing of the resistivity was not small. The reason for the applicability of Eq. (4.1) in a so broad interval of temperatures is not 
clear because according to the results of the renormalization group analysis of Sec. [II.C not the resistivity but the conductivity should obey Eq. (4.1). A more careful experimental study might clarify this question.

The unusual logarithmic behavior of the type, Eq. (4.1), has been observed not only in "standard" granular systems but also in high- $T_{c}$ cuprates at very strong magnetic fields. The first observation of this dependence was done on underdoped $\mathrm{La}_{2-x} \mathrm{Sr}_{x} \mathrm{CuO} \mathrm{O}_{4}$ crystals (Ando et al., 1995). The superconductivity in this experiment was suppressed with pulsed magnetic fields of $61 T$. It was found that both the in-plane resistivity $\rho_{a b}$ and out-of-plane resistivity $\rho_{c}$ diverged logarithmically with decreasing the temperature. This means that a $3 d$ effect was observed in a very strong magnetic field and traditional explanations for a logarithmic behavior like a weak localization or the Kondo effect could not clarify the situation.

In a subsequent publication (Boebinger et al., 1996) a metal-insulator crossover was observed in the same material at a $S r$ concentration near optimum doping $(x \simeq 0.16)$. In underdoped samples both $\rho_{a b}$ and $\rho_{c}$ showed no evidence of saturation at low temperatures and diverged as the logarithm of the temperature. The authors called this state "insulator" in contrast to the state at high doping where the resistivity did not have a pronounced dependence on the temperature. It was conjectured by Boebinger et al. (1996) that the logarithmic behavior they observed might be related to the one seen in the experiment (Simon et al., 1987) on granular Nb. This would demand a phase segregation throughout the underdoped regime of $L S C O$.

We hope that our results for the model of the granular materials may be applicable to the experiments on the $\mathrm{La}_{2-x} \mathrm{Sr}_{x} \mathrm{CuO}$ crystals Ando et al., 1995; Boebinger et al., 1996), which would mean that the underdoped crystals have a granular structure and the logarithmic behavior is due to the Coulomb interaction. The transition to the metallic state of Refs. (Ando et al., 1995; Boebinger et al., 1996) would mean that at higher doping the granularity disappears. A quantitative comparison of Eq. (2.72) with the data of Refs. (Ando et al., 1995; Boebinger et al., 1996) was done in Beloborodov et al. (2003) and a good agreement was found.

What is interesting, a microscopic granularity was directly experimentally observed in the superconducting state of $\mathrm{Bi}_{2} \mathrm{Sr}_{2} \mathrm{CaCu}_{2} \mathrm{O}_{8+\delta}$ by the STM probe (Lang et al., 2002). As this cuprate is rather similar in its structure to those studied in Refs. (Ando et al., 1995; Boebinger et al., 1996) the assumption that the materials studied there were granular does not look groundless.

The logarithmic dependence of the resistivity on temperature has also been observed in many other experiments. For example, in Gerber (1990) this dependence was observed in granular $P b$ films. It was also observed in phase compounds of
$\mathrm{Nd}_{2-x} \mathrm{Ce} e_{x} \mathrm{CuO} \mathrm{O}_{4-y}$ (Radhakrishnan et al., 1990).

\section{Hopping conductivity}

As we have mentioned in the previous subsection, the logarithmic temperature dependence is usually seen in samples with a good coupling between the grains. If the coupling is weak, one observes usually the EfrosShklovskii law, Eq. (2.14).

According to the theory developed in Sec. [II.G the characteristic temperature $T_{0}$ entering Eq. (2.14) is determined by Eqs. (2.111) and (2.116) for the elastic and inelastic co-tunneling, respectively.

Now we want to compare our results for the VRH conductivity of granular metals obtained in Sec. [II.G with the most recent experimental data. In Ref. (Tran et al., 2005) the zero-bias conductivity was studied for bilayers, trilayers, tetralayers and thick films of $A u$ nanoparticles with the particle diameters around $5.5 \mathrm{~nm}$ and their dispersion less than $5 \%$.

Figure 6 demonstrates that the zero-bias conductivity follows $\sigma(T) \sim \exp \left[-\left(T_{0} / T\right)^{1 / 2}\right]$ over the range $30-90 K$ for the multilayers and $30-150 K$ for the thick films. The fits indicated by the lines give $T_{0}=(4.00 \pm 0.02) \times 10^{3} \mathrm{~K}$ and $(3.00 \pm 0.01) \times 10^{3} \mathrm{~K}$ for the multilayers and thick films, respectively.

At temperatures slightly exceeding $100 K$, the conductivity for the multilayers starts deviating from the lowtemperature behavior (dashed lines) and crosses over to Arrhenius behavior $\sigma(T) \sim \exp \left[-U / k_{B} T\right]$ (Fig. 6] inset) with the activation energies $U / k_{B} \approx 320 \pm 8 K$.

Associating this high-temperature behavior with the nearest-neighbor tunneling between the particles we can use $U \approx 0.2 E_{c}$, in analogy with the monolayers (Parthasarathy et al., 2004). This gives us an estimate $E_{c} \approx 1600 K$, where $E_{c}=e^{2} / \tilde{\kappa} a$ is the Coulomb charging energy of an individual grain, expressed in terms of the grain radius $a$, the electron charge $e$, and the dielectric constant of the surrounding medium $\tilde{\kappa}$. The charging energy $E_{c} \approx 1600 K$ for this system leads to $\tilde{\kappa} \approx 4$.

The energy scale $T_{0}$ is related to the localization length $\xi$ by Eqs. (2.111 2.116). Using the above values for $\tilde{\kappa}$ and $T_{0}$, we find $3 \mathrm{~nm}<\xi<4 \mathrm{~nm}$ for both the multilayers and thick films, which corresponds to the localization within one grain. This is an important check because larger values would imply strongly coupled clusters of the grains.

The typical hopping distance $r^{*}(T)$ is given by Eq. ( 2.122). The number of grains $N^{*}$ involved in a typical hop is $N^{*}=r^{*} / d_{0}$, with a center-to-center distance $d_{0} \approx$ $8 \mathrm{~nm}$ between neighboring grains. At $T=10 \mathrm{~K}$ this leads to $N^{*}=4$ for multilayers and $N^{*}=4-5$ for the thick films.

As temperature increases, $N^{*}$ decreases down to $N^{*} \sim$ 1 and this is the point of the crossover to a standard activation transport, Eq. (2.13). For the multilayers, this happens at $T \approx 90-95 K$ but for the thick films only above $T \approx 130 K$. Both the estimates are in excellent 
agreement with the data in Fig. 6]

\section{Negative magnetoresistance}

Let us compare the theoretical predictions on the negative magnetoresistance presented in Sec. III.E with the available experimental results of Gerber et al. (1997). In that work three samples were studied. We will concentrate our attention on the sample 2, Fig. 4, of Gerber et al. (1997) that has a metallic conductivity behavior above the critical temperature.

We analyze the case of low temperatures $T \ll T_{c}$ and magnetic fields $H>H_{c}$, where $T_{c} \approx 1.6 K$ is the critical temperature for $A l$ grains studied in the experiment and $H_{c}$ is the critical magnetic field that suppresses the superconductivity in a single grain, $E_{0}\left(H_{c}\right)=\Delta_{0}$, where $E_{0}(H)$ was defined below Eq. 3.67. At temperature $T \simeq 0.3 K$ and magnetic field $H \simeq 4 T$ this sample shows a large negative magnetoresistance. The resistivity has the maximum at $H=2.5 T$ and the value of this peak is more than twice as large as the resistivity in the normal state (that is, at $H \gg H_{c}$, when all superconducting fluctuations are completely suppressed). A negative magnetoresistance due to weak localization (WL) is typical for disordered metals and in order to describe the experimental data, its value should be estimated along with the effects of the superconducting fluctuations discussed in the previous subsection.

The total conductivity of the granular metal under consideration including effects of WL and superconducting fluctuations can be written in the form:

$$
\sigma=\sigma_{0}+\delta \sigma_{D O S}+\delta \sigma_{A L}+\delta \sigma_{M T}+\delta \sigma_{W L}
$$

At low temperatures, $T \ll T_{c}$, the contribution $\delta \sigma_{D O S}$ originating from the reduction of DOS due to the formation of the virtual Cooper pairs is larger than the contributions $\delta \sigma_{A L}$ and $\delta \sigma_{M T}$ since the latter vanish in the limit $T \rightarrow 0$. So, let us concentrate on estimating the contributions $\delta \sigma_{D O S}$ and $\delta \sigma_{W L}$.

It is not difficult to show that in the case under consideration the weak localization corrections originating from a contribution of Cooperons are strongly suppressed by the magnetic field. Using approximations developed by Beloborodov and Efetov (1999) one can easily obtain the following expression for a $3 d$ cubic lattice of metallic grains at tunneling conductances $g \ll E_{0}(H) / \delta$

$$
\frac{\delta \sigma_{W L}}{\sigma_{0}} \sim-\frac{1}{g}\left[\frac{\Gamma}{E_{0}(H)}\right]^{2}
$$

Equation (4.3) shows that the weak localization correction in the strong magnetic fields $H>H_{c}$ considered here is always small. Comparing Eq. (4.3) with Eq. (3.66) for DOS correction one can see that in the limit $g \ll \Delta_{0} / \delta$ the contribution from the weak localization correction can be neglected.
Now, let us estimate the corrections $\delta \sigma_{D O S}$ and $\delta \sigma_{W L}$ using the parameters of the experiment (Gerber et al., 1997). For the typical diameter $120 \pm 20 \AA$ of $A l$ grains studied by Gerber et al. (1997), the mean level spacing $\delta$ is approximately $\delta \approx 1 K$. Using the critical temperature $T_{c} \simeq 1.6 \mathrm{~K}$ for $A l$ we obtain for the BCS gap in a single grain the following result $\Delta_{0} \approx 1.8 T_{c} \approx 3 K$. Substituting the extracted values of the parameters into Eq. (3.66) we can estimate the maximal increase of the resistivity. As a result, we obtain $\left(\delta \rho / \rho_{0}\right)_{\max } \approx 0.4$, which is somewhat smaller but not far from the value $(\delta \rho / \rho)_{\exp } \approx 1$ observed experimentally.

Although Eq. (3.66) gives smaller values of $\left(\delta \rho / \rho_{0}\right)_{\max }$ than the experimental ones, the discrepancy cannot be attributed to the weak localization effects. Using the experimental values of the conductance $g$, mean level spacing $\delta$ and the superconducting gap $\Delta_{0}$ we find from Eq. (4.3) that $\delta \sigma_{W L}$ is 10 times smaller than $\delta \sigma_{D O S}$. The value of the correction $\delta \sigma_{W L} / \sigma_{0}$, Eq. (4.3), near $H_{c}$ equals $2.8 \times 10^{-2}$.

At the same time, we should not take this disagreement too seriously because all the results were obtained under the assumption of a large conductance $g \gg 1$, while experimentally this parameter is not large. As the experimental value of $\delta \sigma / \sigma_{0}$ is not small, we come again to the conclusion that the parameters of the system are such that the Eq. (3.66) is no longer applicable and one should develop a more sophisticated theory to describe this region more accurately.

\section{B. Outlook}

We discussed in this review a rather simple general model that allowed us to understand many properties of granular materials. This is a model of disordered or chaotic grains coupled to each other by the electron tunneling. A very important ingredient of the model is the long range part of the Coulomb interaction taken in the form of the charging energy of the grains. For the description of the superconducting grains we included the superconducting BCS gap into the consideration.

We considered the limit of temperatures $T$ exceeding the mean level spacing $\delta$ in the grains and neglected quantum size effects. Considering the superconducting grains we assumed that the superconducting gap $\Delta$ was larger than $\delta$. These regimes are most easily achieved experimentally and this was the main reason for choosing them.

In spite of its simplicity, a variety of interesting phenomena has been derived from the model involved. Such phenomena as the logarithmic dependence of the conductivity on temperature or Efros-Shklovskii law for a regular system of grains with an almost perfect shape remained unexplained for several decades and have been clarified within the model only recently. Another interesting effect concerns the negative magnetoresistance due to superconducting fluctuations. For these effects we have obtained not only qualitative but a good quantita- 
tive description.

The granularity is a rather general phenomenon and it may be encountered rather unexpectedly. Thin metallic films are often rather granular than homogeneously disordered, (Gantmakher et al., 1996; Goldman and Marković, 1998). Another unexpected conclusion about the granularity of some underdoped high $T_{c}$ cuprates has been arrived at using STM technique, (Lang et al., 2002).

Studying the granular systems may help to understand some properties of homogeneously disordered metals and superconductors. As all scales involved in the model we studied are much larger than the electron wavelength, it might in some cases simplify explicit calculations. This concerns, in particular, study of the regime of $g \sim 1$. We presented arguments that the metalinsulator transition is possible at least in three dimensions. The superconductor-insulator transition is also possible. Studying these transitions is the most challenging extension of the present study but this may be simpler than investigation of strongly disordered systems with interaction.

From the experimental side, an evident extension of the works presented here is fabricating grains made of ferromagnets and studying properties of such systems. One can couple these grains directly or put them into normal metals or superconductors. One can make superconducting grains and embed them into normal metals or not very strong ferromagnets, etc. All these systems promise very unusual properties.

One of the examples of an unexpected behavior in the system of ferromagnetic grains imbedded in a superconductor is inducing a magnetic moment in the superconductor over large distances of the order of the size of the Cooper pairs. The direction of this magnetic moment is opposite to that of the ferromagnet and one comes to a phenomenon of the "spin screening" (Bergeret et al., 2004, 2005). Different directions of the magnetic moments of the grains may lead to the phenomenon of an "odd triplet" superconductivity, (Bergeret et al., 2001, 2005).

The list of new theoretical and experimental possibilities that are anticipated in granular systems can be continued. Taking into account the growing number of existing and potential industrial applications of the granular materials we are confident that all this is only the beginning of an exciting development.

\section{Acknowledgements}

We thank Igor Aleiner, Anton Andreev, Mikhail Feigel'man, Alexander Finkel'stein, Frank Hekking, Alexei Koshelev for useful discussions. We especially grateful to Anatoly Ivanovich Larkin for invaluable discussions. We thank Thu Tran, Heinrich Jaeger, Xiao-Min Lin for continues communication of relevant experimental data and stimulating discussions. K. E. thanks SFB
Transregio 12 of DFG and DIP for a financial support.

\section{APPENDIX A: Calculation of the tunneling probability $P_{e l}$ in the elastic regime, Eq. (2.105)}

In this appendix we derive the probability of elastic electron tunneling $P_{e l}$ between two distant grains through a chain of other grains, Eq. 2.105. Such a probability can be found comparatively easily only for the model with the diagonal Coulomb interaction $E_{i j}^{c}=$ $E_{i} \delta_{i j}$. For this reason we consider first such a simplified model and then discuss the generalization of the results obtained for the case of realistic capacitance matrices $E_{i j}^{c}$ with nonzero off-diagonal elements.

In the model with the diagonal Coulomb interaction, the electron-hole excitation energies $E_{i}^{ \pm}$that will enter the final results for the hopping probability are given by

$$
E_{i}^{ \pm}=E_{c}^{i}-V_{i},
$$

where $V_{i}$ is the local potential that models the presence of the electrostatic disorder. These energies have to be strictly positive and larger than the energies of the initial and final states otherwise the tunneling path could have been cut into two or more independent parts. This allows us to assume that the temperature is less than all $E_{i}^{ \pm}$. For this reason we will consider the probability of the elastic process in the limit $T \rightarrow 0$.

The tunneling probability $P$ between the sites $i_{0}$ and $i_{N+1}$ is proportional to the square of the absolute value of the amplitude $A_{i_{0}, i_{N+1}}$ of the corresponding tunneling process

$$
A_{i_{0}, i_{N+1}}=<0\left|\hat{c}_{i_{N+1}} \hat{S} \hat{c}_{i_{0}}^{+}\right| 0>.
$$

Here $\hat{S}$ is the evolution operator written in the interaction representation that takes into account only the tunneling part of the Hamiltonian (2.15). The conjugate amplitude $A^{*}$ can be written as the probability of the inverse process of the tunneling between $i_{N+1}-$ st and $i_{0}$-th grains

$$
A_{i_{0}, i_{N+1}}^{*}=A_{i_{N+1}, i_{0}}=<0\left|\hat{c}_{i_{0}} \hat{S} \hat{c}_{i_{N+1}}^{+}\right| 0>.
$$

The probability of the tunneling process $P=A^{*} A$ can be found by perturbative expansion of the amplitude in the tunneling matrix elements $t_{i j}$ and further averaging over the realizations of $t_{i j}$. At the same time the Coulomb interaction cannot be considered perturbatively and should be taken into account exactly. In order to construct a proper perturbative expansion we use the gauge transformation described in Sec. II.B.3 that allows us to transfer the strong Coulomb interaction into the phase factors that accompany the tunneling matrix elements (see Eqs. 2.42 2.43).

Fluctuations of the phases $\varphi_{i}(\tau)$ are governed by the Coulomb action (2.56) and we obtain the following expression for the correlation function $\Pi(\tau)$ that plays a 


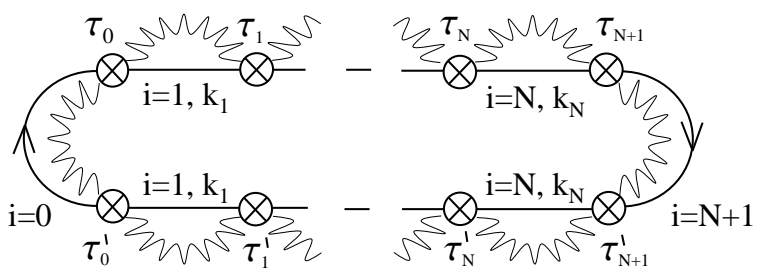

FIG. 22 This diagram represents the tunnelling probability via elastic co-tunneling processes. The crossed circles represent the tunnelling matrix elements $t_{k, k^{\prime}}^{i j} e^{i \phi_{i}(\tau)} e^{-i \phi_{j}(\tau)}$ where phase factors appear from the gauge transformation. Wavy lines represent the average of the phase factors $\left\langle e^{i \phi\left(\tau_{1}\right)} e^{-i \phi\left(\tau_{2}\right)}\right\rangle$ with respect to the Coulomb action.

very important role for a description of the insulating state (c.f. Eq. (3.7))

$$
\begin{aligned}
\Pi\left(\tau_{1}-\tau_{2}\right) & \equiv\left\langle\exp \left(i \phi_{i}\left(\tau_{1}\right)-i \phi_{i}\left(\tau_{2}\right)\right)\right\rangle \\
& =\exp \left(-E_{c}^{i}\left|\tau_{1}-\tau_{2}\right|-V_{i}\left(\tau_{1}-\tau_{2}\right)\right) .
\end{aligned}
$$

Since we are interested in the optimal tunneling path we will consider only trajectories with no return points (no loops). This simplifies the consideration substantially because the phases on different sites are not correlated for the diagonal model under consideration.

The gauge transformation approach allows us to represent the tunneling probability $P$ by the diagram shown in Fig. 22 In order to simplify the derivation we work now using the basis of the exact eigenstates of the single particle Green functions that automatically takes into account the presence of the disorder within each grain. An alternative procedure based on the momentum representation leading to the same results would require the averaging over disorder in each grain and inclusion of the diffusion propagators. The Green function lines on the initial $i_{0}$ and final $i_{N+1}$ grains describe the processes for a particle located on these sites, while the intermediate Green function lines represent the tunneling amplitudes $A$ (upper part) and $A^{*}$ (lower part). The wavy lines denote the correlation function (A4) that takes into account the Coulomb correlations.

It may seem surprising that the two wavy lines that belong to the same $i$-th site are consider independently, i.e. that four exponent correlation function is factorized in a certain way into the two second order ones. One can check however that this factorization is justified indeed as long as the time intervals $\left(\tau_{i-1}, \tau_{i}\right)$ and $\left(\tau_{i-1}^{\prime}, \tau_{i}^{\prime}\right)$ do not overlap.

This factorization can most easily be understood by presenting the correlation function of $N$ phase exponents as the exponent of the interaction energy of $N$ charges interacting via a linear one dimensional Coulomb potential. The two nearby charges with the opposite signs create an "electric field" only in the region between the two. Thus, the energy of the dipoles can be considered independently as long as these dipoles do not overlap geometrically. For a detailed discussion of the Coulomb analogy we refer the reader to Beloborodov et al. (2005c).
It follows from this discussion that all the electron Green functions in the diagram Fig. 22 are accompanied by the correlation function $\Pi(\tau)$. For this reason, it is convenient to introduce a modified Green function $\tilde{G}(\tau)$ in the following way

$$
\tilde{G}(\tau)=G(\tau) \Pi(\tau) .
$$

The Green function $\tilde{G}(\tau)$ has a very simple form in the time representation: For positive arguments the Green function describes the electron excitation (Abrikosov et al., 1965)

$$
\tilde{G}_{\xi_{i}}(\tau>0)=\left[n\left(\xi_{i}\right)-1\right] e^{-\xi_{i} \tau-E_{i}^{+} \tau},
$$

where $\xi_{i}$ is the bare single particle electron energy counted with respect to the Fermi energy, Eq. (3.2) and $n\left(\xi_{i}\right)$ is the Fermi distribution function. One can see that the Coulomb part of the electron excitation energy $E_{i}^{+}$ appears naturally in addition to the single particle energy $\xi_{i}$. For negative time arguments the Green function $\tilde{G}$ describes the hole excitation and is given by

$$
\tilde{G}_{\xi_{i}}(\tau<0)=n\left(\xi_{i}\right) e^{-\xi_{i} \tau+E_{i}^{-} \tau},
$$

where $\xi_{i}<0$ is the bare electron energy counted with respect to the Fermi energy and $E_{i}^{-}$is the Coulomb part of the hole excitation energy.

Calculating the diagram in Fig. 222 we further note that the intermediate time intervals are of the order of the inverse Coulomb energy: $\left|\tau_{i+1}-\tau_{i}\right|,\left|\tau_{i+1}^{\prime}-\tau_{i}^{\prime}\right| \sim E_{c}^{-1}$. In the limit of strong Coulomb interaction $\left(E_{c} \gg \delta\right)$ considered here, we can integrate over the intermediate times independently. Each intermediate block gives rise to the factor

$$
P_{k}=\frac{\Gamma_{k}}{2 \pi} \int_{-\infty}^{\infty} d \xi \int d \tau_{1} d \tau_{2} \tilde{G}_{\xi}\left(\tau_{1}\right) \tilde{G}_{\xi}\left(\tau_{2}\right)=\frac{\Gamma_{k}}{\pi \tilde{E}_{k}}
$$

where the energy $\tilde{E}_{k}$ is the combination of the energies $E_{k}^{+}$and $E_{k}^{-}$defined by Eq. (2.107) and $\Gamma_{k}=g_{k} \delta_{k}$, with $g_{k}$ being the conductance between $k$-th and $k+1-$ st grains. We note that in the insulating system under consideration the energy scale $\Gamma$ cannot be any more interpreted as the escape rate, nevertheless its introduction is convenient even in this regime.

In order to determine the time dependence of the tunneling process we notice that the time intervals $\tau_{0}^{\prime}-\tau_{0}$ and $\tau_{N+1}^{\prime}-\tau_{N+1}$ coincide within the accuracy of the inverse charging energy. In the limit of the strong Coulomb interaction one can take both the intervals to be equal to the "instanton" time $\tau$ that electron spends out of its original place at $i=0$. Thus, the time dependence of the tunneling process is simply given by

$$
e^{-\left(\varepsilon_{N+1}-\varepsilon_{0}\right) \tau},
$$

where $\varepsilon_{N+1}$ and $\varepsilon_{0}$ are the electron and hole excitation energies on the cites $N+1$-st and 0 -th respectively. Making an analytical continuation to real times $\tau=i t$ and taking the last integral over $t$ we obtain the delta function

$$
2 \pi \delta\left(\varepsilon_{N+1}-\varepsilon_{0}\right),
$$


that indeed shows that the process is elastic, i.e. electron can tunnel only to the state with exactly the same energy.

Finally, we obtain for the tunneling probability of the elastic process

$$
P_{e l}=w \delta\left(\varepsilon_{N+1}-\varepsilon_{0}\right) g_{0} \prod_{k=1}^{N} P_{k},
$$

where the factor $w=n\left(\xi_{0}\right)\left[1-n\left(\xi_{N+1}\right)\right]$ takes into account the occupation numbers of the initial and final states.

We see that the total probability $P_{e l}$ contains the product of the ratios $\Gamma_{i} / \tilde{E}_{i}$. For this reason it is convenient to introduce the geometrical averages of theses quantities along the tunneling path that were introduced in Sec. II.G.2 Writing the total probability $P_{e l}$ in terms of the quantities $\bar{\Gamma}, \bar{E}$ we obtain the result (2.105).

The factorization of the probability $P_{e l}$, Eq. (A11), into the product of the probabilities $P_{k}$ means that the hops from grain to grain are independent from each other, which is a consequence of the diagonal form of the Coulomb interaction.

If the Coulomb interaction is so long ranged that it involves many granules, the situation is more complicated since the integrals over the time variables cannot be taken on each site independently. Nevertheless, one can generalize the obtained results to the case of the long range Coulomb interaction as follows: The most important effect of the off diagonal part of the interaction $E_{c}^{i j}$ is the renormalization of the excitation energies. Since the final result contains the single particle excitations only, this effect may be included by the proper definition of the electron-hole excitation energies

$$
E_{i}^{ \pm}=E_{i i}-\mu_{i}
$$

where $\mu_{i}$ represents the local potential formed by both the external potential $V_{i}$ and all charges surrounding the grain $i$. This procedure, however, does not include all the effects of the presence of the off diagonal part of $E_{c}^{i j}$. The problem is that the virtual process represented by the diagram in Fig. 22 does not correspond to a causal classical process. For this reason the virtual field created by an electron $i$ at a time $t_{i}$ affects in general the same electron when it is present on the other site at a different time.

However, such an interaction decays at least as $1 / r^{2}$ with the distance. This assumes that the long range effects of the Coulomb interaction are not important, since the corresponding integral along the chain converges. Thus, all the effects that are not taken into account by the substitution A12 are short range and may only lead to the change of a constant under the logarithm of the effective localization length (2.109). Using the analogy with classical Coulomb problem (Beloborodov et al. , 2005c) one can estimate the boundaries for the factor under the logarithm as $0.5 \lesssim c<1.0$.
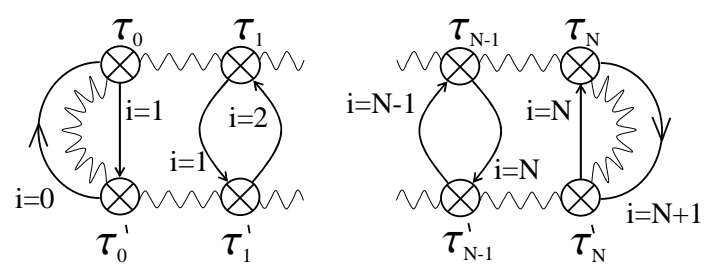

FIG. 23 This diagram represents the tunnelling probability via inelastic cotunneling processes. The crossed circles represent the tunnelling matrix elements $t_{k, k^{\prime}}^{i j} e^{i \phi_{i}(\tau)} e^{-i \phi_{j}(\tau)}$ where phase factors appear from the gauge transformation. Wavy lines represent the average of the phase factors $\left\langle e^{i \phi\left(\tau_{1}\right)} e^{-i \phi\left(\tau_{2}\right)}\right\rangle$ with respect to the Coulomb action.

\section{APPENDIX B: Calculation of the tunneling probability $P_{i n}$ in the inelastic regime, Eq. (2.114)}

In this appendix we derive the probability of the inelastic cotunneling $P_{i n}$ through a chain of grains. As in the case of the elastic processes considered in the previous section, the probability of the inelastic processes can easily be found for the model with the diagonal Coulomb interaction. For this model, we follow the same steps as previously, making the gauge transformation described in Sec.II.B.3 and expanding the tunneling probability in the tunneling matrix elements.

The diagram describing the inelastic process is shown in Fig. 23 We see that it consists only of short electron loops, which means that the charge on each step is transferred by different electrons as it should be in the inelastic process.

All intermediate Green functions with the grain index $i=0, \ldots N$ enter the diagram in Fig. 23 being integrated over the internal states of the grain, such that each Green function is

$$
G(\tau) \equiv \sum_{k} G_{k}(\tau)=\frac{\pi T}{\sin (\pi T \tau)} .
$$

The processes for the electrons located on the initial and final sites $\left(i_{0}\right.$ and $\left.i_{N}\right)$ are given by the Green functions that are accompanied by the phase correlations $\Pi(\tau)$ exactly in the same way as in the case of the elastic cotunneling and, thus, can be described in terms of the Green functions $\tilde{G}$.

The intervals $\tau_{0}-\tau_{0}^{\prime}$ and $\tau_{N}-\tau_{N}^{\prime}$ coincide with each other within the accuracy of the inverse Coulomb energy and we just put $\tau_{0}-\tau_{0}^{\prime} \approx \tau_{N}-\tau_{N}^{\prime} \equiv \tau$. In the same limit, the integrals over the intermediate times intervals $\Delta \tau_{k}=\tau_{k}-\tau_{k-1}$ (and over $\tau_{k}^{\prime}-\tau_{k-1}^{\prime}$ ) can be calculated independently resulting in the contribution

$$
\int_{-\infty}^{\infty} d\left(\Delta \tau_{k}\right) e^{-E_{k}^{c}\left|\Delta \tau_{k}\right|+\mu_{k} \Delta \tau_{k}}=2 / \tilde{E}_{k}
$$

where the energy $\tilde{E}_{k}$ is defined in Eq. (2.107). Collecting all the terms for the probability $P_{i n}$ one obtains

$$
P_{i n}(\tau)=\frac{\bar{g}^{N+1}}{2 \pi^{N+1}}\left(\frac{2 \pi T}{\bar{E} \sin (\pi T \tau)}\right)^{2 N} e^{-\tau \Delta \varepsilon},
$$


where $\Delta \varepsilon=\varepsilon_{N+1}-\varepsilon_{0}$ is the difference between the energies of the initial and final states and $\bar{E}$ and $\bar{g}$ are geometrical averages along a the tunneling path (see Eqs. (2.106).

Finally, in order to find the tunneling probability $P_{\text {in }}$ for the inelastic cotunneling one has to make the analytical continuation in Eq. (B3) to the real times $\tau_{w}=i t_{w}$ and integrate over $t_{w}$ arriving at

$$
P_{i n}=\frac{w g^{N+1}}{2 \pi^{N+1}} \int_{-\infty}^{+\infty} d t\left[\frac{2 \pi i T}{\bar{E} \sinh (\pi T t)}\right]^{2 N} e^{-i t \Delta \varepsilon} .
$$

Here the singularity of the function $\sinh ^{-2 N}(\pi T t)$ is assumed to be in the upper half of the complex plain. At zero temperature one can easily calculate the integral over the variable $t$ in Eq. (B4) by shifting the contour of integration in the complex plain either to $-i \infty$ for positive $\Delta \varepsilon$ or to $+i \infty$ for negative $\Delta \varepsilon$.

In the first case we obtain $P_{i n}=0$. This reflects the fact that the real tunneling process with an increase of the energy of the electron is forbidden at $T=0$. In the latter case, $\Delta \varepsilon<0$, the zero temperature probability is determined by the pole of the function $\sinh ^{-2 N}(\pi T t)$ that results in Eq. (2.118).

At finite temperatures the integral in Eq. (B) can be expressed in terms of the Euler Gamma functions that leads to the general expression (2.114).

Generalization of the result to include the long-range interaction consists, as in the case of the elastic cotunneling, of a proper redefinition (A12) of the electron/hole excitation energies. Considerations analogous to those presented in the previous appendix allow us to conclude that inclusion of the off diagonal terms in the capacitance matrix results only in the appearance of the coefficient under the logarithm (Beloborodov et al., 2005c) in the expression for the localization length (2.117).

\section{References}

Abeles, B., 1977, Phys. Rev. B 15, 2828.

Abeles, B., P. Sheng, M. Coutts, and Y. Arie, 1975, Adv. Phys. 24, 407.

Abrahams, E., P. Anderson, D. Licciardello, and T. Ramakrishnan, 1979, Phys. Rev. Lett. 42, 673.

Abrahams, E., S. V. Kravchenko, and M. P. Sarachik, 2001, Rev. Mod. Phys. 73, 251.

Abrahams, E., M. Redi, and J. Woo, 1970, Phys. Rev. B 1, 208.

Abrikosov, A., 1988, Fundamentals of the Theory of Metals (North Holland, Amsterdam).

Abrikosov, A. A., and L. P. Gor'kov, 1961, Sov. Phys. JETP 12, 1243.

Abrikosov, A. A., L. P. Gor'kov, and I. E. Dzyaloshinski, 1965, Methods of quantum field theory in statistical physics (Dover Publications, Inc. New York).

Aleiner, I. I., P. W. Brouwer, and L. I. Glazman, 2002, Phys. Rep. 358, 309.

Alhassid, Y., 2000, Rev. Mod. Phys. 72, 895.
Altland, A., L. Glazman, and A. Kamenev, 2004, Phys. Rev. Lett. 92, 026801.

Altland, A., L. I. Glazman, A. Kamenev, and J. S. Meyer, 2005, cond-mat/0507695

Altshuler, B., and A. Aronov, 1985, Electron-Electron Interaction in Disordered Conductors (North-Holland, Amsterdam), volume 10 of Modern Problems in Condensed Matter Science, p. 1.

Altshuler, B. L., A. G. Aronov, and D. E. Khmel'nitskii, 1982, J. Phys. C 15, 7367.

Altshuler, B. L., D. E. Khmelnitskii, A. I. Larkin, and P. A. Lee, 1980, Phys. Rev. B 22.

Ambegaokar, V., and A. Baratoff, 1963, Phys. Rev. Lett. 10, 486.

Ambegaokar, V., U. Eckern, and G. Schön, 1982, Phys. Rev. Lett. 48, 1745.

Anderson, P. W., 1958, Phys. Rev. 109, 1492.

Anderson, P. W., 1959, J. Phys. Chem. Solid 1, 26.

Anderson, P. W., 1964, in Lectures on the Mony-Body Problems, volume 2 (Academic, New York).

Ando, Y., G. Boebinger, A. Passner, T. Kimura, and K. Kishio, 1995, Phys. Rev. Lett. 75, 4662.

Andreev, A. V., and I. S. Beloborodov, 2004, Phys. Rev. B 69, 081406(R).

Aslamazov, L. G., and A. I. Larkin, 1968, Fiz. Tverd. Tela Leningrad , 1104[Sov. Phys. Solid State 10, 875 (1968)].

Averin, D., and Y. Nazarov, 1992, in Single Charge Tunneling (Plenum, New York), volume 294.

Averin, D. A., and Y. V. Nazarov, 1990, Phys. Rev. Lett. 65, 2446.

Averin, D. V., and K. K. Likharev, 1991, in Mesoscopic Phenomena in Solids (Elsevier, North-Holland, Amsterdam), p. 173.

Basko, D., I. Aleiner, and B. Altshuler, 2005, condmat/0506617.

Beenakker, C. W. J., 1997, Rev. Mod. Phys. 69, 731.

Belitz, D., and T. R. Kirkpatrick, 1994, Rev. Mod. Phys. 66, 261.

Beloborodov, I. S., and K. B. Efetov, 1999, Phys. Rev. Lett. $\mathbf{8 2}, 3332$.

Beloborodov, I. S., K. B. Efetov, A. Altland, and F. W. J. Hekking, 2001, Phys. Rev. B 63, 115109.

Beloborodov, I. S., K. B. Efetov, and A. I. Larkin, 2000, Phys. Rev. B. 61, 9145.

Beloborodov, I. S., K. B. Efetov, A. V. Lopatin, and V. M. Vinokur, 2003, Phys. Rev. Lett 91, 246801.

Beloborodov, I. S., K. B. Efetov, A. V. Lopatin, and V. M. Vinokur, 2005a, Phys. Rev. B 71, 184501.

Beloborodov, I. S., Y. Fominov, A. V. Lopatin, and V. M. Vinokur, 2006, cond-mat/0509386.

Beloborodov, I. S., A. V. Lopatin, F. J. W. Hekking, R. Fazio, and V. M. Vinokur, 2005b, Europhys. Lett. 69, 435.

Beloborodov, I. S., A. V. Lopatin, G. Schwiete, and V. M. Vinokur, 2004a, Phys Rev B 70, 073404.

Beloborodov, I. S., A. V. Lopatin, and V. M. Vinokur, 2004b, Phys. Rev. B 70, 205120.

Beloborodov, I. S., A. V. Lopatin, and V. M. Vinokur, 2004c, Phys. Rev. Lett. 92, 207002.

Beloborodov, I. S., A. V. Lopatin, and V. M. Vinokur, 2005c, Phys. Rev. B 72, 125121.

Bergeret, F. S., A. F. Volkov, and K. B. Efetov, 2001, Phys. Rev. Lett. 86, 4096.

Bergeret, F. S., A. F. Volkov, and K. B. Efetov, 2004, Euro- 
phys. Lett. 66, 111.

Bergeret, F. S., A. F. Volkov, and K. B. Efetov, 2005, Rev. Mod. Phys. 77, 1321.

Biagini, C., T. Caneva, V. Tognetti, and A. A. Varlamov, 2005a, Phys. Rev. B 72, 041102.

Biagini, C., R. Ferone, R. Fazio, F. W. J. Hekking, and V. Tognetti, 2005b, Phys. Rev. B 72, 134510.

Black, C., D. Ralph, and M. Tinkham, 1996, Phys. Rev. Lett. 76, 688 .

Boebinger, G., Y. Ando, A. Passner, T. Kimura, M. Okuya, J. Shimoyama, K. Kishio, K. Tamasaku, N. Ichikawa, and S. Uchida, 1996, Phys. Rev. Lett. 77, 5417.

Bulgadaev, S., 1984, Pis'ma Zh. Eksp. Teor. Phys. 39, 264, [Sov. Phys. JETP Lett. 39, 314 (1985)].

Caldeira, A. O., and A. J. Leggett, 1981, Phys. Rev. Lett. 46, 211.

Caldeira, A. O., and A. J. Leggett, 1983, Ann. Phys. 149, 374.

Chakravarty, S., G. L. Ingold, and A. L. S. Kivelson, 1986, Phys Rev Lett 56, 2303.

Chakravarty, S., S. Kivelson, G. Zimanyi, and B. Halperin, 1987, Phys Rev B 35, 7256.

Chervenak, J. A., and J. J. M. Valles, 2000, Phys Rev B 61, R9245.

Clogston, A. M., 1960, Phys. Rev. Lett. 5, 464.

Cohen, M., L. Falicov, and J. Phillips, 1962, Phys. Rev. Lett. $\mathbf{8}, 316$.

Collier, C., T. Vossmeyer, and J. Heath, 1998, Annual review of Physical Chemistry 49, 371.

Collier, C. P., R. J. Saykally, J. J. Shiang, S. E. Henrichs, and J. R. Health, 1997, Science 277, 1978.

Davidović, D., and M. Tinkham, 1999, Phys. Rev. Lett. 83, 688.

von Delft, J., A. D. Zaikin, D. S. Golubev, and W. Tichy, 1996, Phys. Rev. Lett. 77, 3189.

Du, H., C. L. Chen, R. Krishnan, T. D. Krauss, J. M. Harbold, F. W. Wise, M. G. Thomas, and J. Silcox, 2002, Nano Lett 2, 1321

Eckern, U., G. Schön, and V. Ambegaokar, 1984, Phys. Rev. B 30, 6419.

Efetov, K., 1983, Adv. Phys, 32, 53.

Efetov, K. B., 1980, Zh. Eksp. Teor. Fiz. 78, 2017, [Sov. Phys. JETP.

Efetov, K. B., 1997, Supersymmetry in Disorder and Chaos (Cambridge University Press, New York).

Efetov, K. B., A. I. Larkin, and D. E. Khmel'nitskii, 1980, Zh. Eksp. Teor. Fiz. 79, 1120, [Sov. Phys. JETP 52, 568 (1980)].

Efetov, K. B., and A. Tschersich, 2002, Europhys. Lett. 59, 114.

Efetov, K. B., and A. Tschersich, 2003, Phys. Rev. B 67, 174205.

Efros, A. L., and B. I. Shklovskii, 1975, J. Phys. C 8, L49.

Falci, G., G. Schön, and G. Zimanyi, 1995, Phys. Rev. Lett. $\mathbf{7 4}, 3257$.

Fazio, R., and H. van der Zant, 2001, Phys. Rep. 355.

Feigel'man, M. V., and A. S. Ioselevich, 2005, Pis'ma Zh. Eksp. Teor. Fiz. 81, 341, [Sov. Phys. JETP Lett. 81, 227 (2005)].

Finkelstein, A. M., 1987, Pis'ma Zh. Eksp. Teor. Fiz. 45, 37, [Sov. Phys. JETP Lett. 45, 46 (1987)].

Finkelstein, A. M., 1990, Electron liquid in Disordered Conductors (Soviet Scientific Reviews, Harwood, London), volume 14
Finkelstein, A. M., 1994, Physica B 197, 636.

Fisher, M. P. A., 1986, Phys. Rev. Lett. 57, 885.

Fisher, M. P. A., 1990, Phys. Rev. Lett. 65, 923.

Fisher, M. P. A., G. Grinstein, and S. M. Girvin, 1990, Phys. Rev. Lett. 64, 587.

Frydman, A., O. Naaman, and R. C. Dynes, 2002, Phys. Rev. B 66, 052509 .

Fujimori, H., S. Mitani, S. Ohnuma, T. Ikeda, T. Shima, and T. Matsumoto, 1994, Mater. Sci. Eng. A 181, 897.

Gantmakher, V. F., M. Golubkov, J. G. S. Lok, and A. Geim, 1996, Zh. Eksp. Teor. Fiz. 82, 958, [JETP 82, 951 (1996)].

Gaponenko, S., 1998, Optical Properties of Semiconductor Nanocrystals (Cambridge, University Press).

Gerber, A., 1990, J. Phys.: Cond. Matter 2, 8161.

Gerber, A., A. Milner, G. Deutscher, M. Karpovsky, and A. Gladkikh, 1997, Phys. Rev. Lett. 78, 4277.

Goldman, A. M., and N. Marković, 1998, Physics Today 51, 39.

Gordon, R., 2000, MRS Bull. 25, 52.

Gorkov, L. P., A. L. Larkin, and D. E. Khmel'nitskii, 1979, Pis'ma Zh. Eksp. Teor. Fiz. 30, 248, [Sov. Phys. JETP Lett. 30, 228 (1979)].

Guinea, F., and G. Schön, 1986, Europhys. Lett. 1, 585.

Hadacek, N., M. Sankquer, and J. C. Villégier, 2004, Phys. Rev. B 69, 024505.

Halperin, W. P., 1986, Rev. Mod. Phys. 58, 533.

Ishida, H., and R. Ikeda, 1998, J. Phys. Soc. Jpn. 67, 983.

Jaeger, H., D. Haviland, B. Orr, and A. Goldman, 1989, Phys Rev B 40, 182.

Jaeger, H. M., D. B. Haviland, A. M. Goldman, and B. G. Orr, 1986, Phys. Rev. B 34, 4920.

Jha, S., and A. A. Middleton, 2005, cond-mat/0511094 .

Kamenev, A., and A. Andreev, 1999, Phys. Rev. B 60, 2218.

Kee, H. Y., I. L. Aleiner, and B. L. Altshuler, 1998, Phys Rev B 58, 5757.

Kim, J. S., M. Granström, R. Friend, N. Johansson, W. Salaneck, R. Daik, W. Feast, and F. Cacialli, 1998, J. Appl. Phys. 84, 6859.

Kopnin, N. B., 2001, Theory of Nonequilibrium Superconductivity (Oxford University Press, Oxford).

Kosterlitz, J., 1976, Phys. Rev. Lett. 37, 1577.

Kravchenko, S. V., and M. P. Sarachik, 2004, Rep. Prog. Phys. 67, 1.

Lang, K. M., V. K. M. Lang, V. Madhavan, E. W. H. J. E. Hoffman, H. Eisaki, S. Uchida, and J. C. Davis, 2002, Nature 415, 412.

Larkin, A. I., 1965, Zh. Eksp. Teor. Fiz. 48, 232, [Sov. Phys. JETP 21, 153 (1965)].

Larkin, A. I., 1999, Ann. Phys. 8, 785.

Larkin, A. I., and Y. N. Ovchinnikov, 1983, Phys. Rev. B 28, 6281.

Larkin, A. I., and A. Varlamov, 2005, Theory of fluctuations in superconductors (Oxford University Press, New York).

Lee, P. A., and T. V. Ramakrishnan, 1985, Rev. Mod. Phys. $\mathbf{5 7}, 287$.

Liao, Z. M., J. Xun, and D. P. Yu, 2005, Phys. Lett. A 345, 386.

Lin, X., H. M. Jaeger, C. Sorensen, and K. Klabunde, 2001, J. Phys. Chem. B 105, 3353.

Liu, Y., D. B. Haviland, B. Nease, and A. Goldman, 1993, Phys. Rev B 47, 5931.

Loh, Y. L., and V. Tripathi, 2006, Phys. Rev. Lett. 96, 046805 .

Loh, Y. L., V. Tripathi, and M. Turlakov, 2005, Phys. Rev. 
B 72, 233404 .

Lukyanov, S., and A. Zamolodchikov, 2004, Journal of Stat. Mechanics, P05003.

Maekawa, S., H. Ebisawa, and H. Fukuyama, 1983, J. Phys. Soc. Jpn. 52, 1352.

Maekawa, S., and H. Fukuyama, 1982, J. Phys. Soc. Jpn. 51, 1380.

Maki, K., 1968a, Prog. Theor. Phys. 39, 897.

Maki, K., 1968b, Prog. Theor. Phys. 40, 193.

Marković, N., C. Christiansen, A. M. Mack, W. H. Huber, and A. M. Goldman, 1999, Phys. Rev. B 60, 4320.

Matveev, K. A., and A. I. Larkin, 1997, Phys. Rev. Lett. 78, 3749.

McLean, W., and M. Stephen, 1979, Phys. Rev. B 19.

Mehta, M., 1991, Random Matrices (Academic Press, San Diego).

Meyer, J. S., A. Kamenev, and L. I. Glazman, 2004, Phys. Rev. B 70, 045310.

Middleton, A. A., and N. S. Wingreen, 1993, Phys. Rev. Lett 71, 3198 .

Mowbray, D. J., and M. S. Skolnick, 2005, J. Phys. D-Applied Physics 38, 2059.

Muller, M., and L. B. Ioffe, 2004, Phys. Rev. Lett. 93, 256403.

Murray, C., C. Kagan, and M. Bawendi, 2000, Annual review of Materials Science 30, 545.

Murray, C. B., D. J. Norris, and M. G. Bawendi, 1993, J. Am. Chem. Soc. 115, 8706.

Nagaev, E., 1992, Physics Reports, 222, 199.

Narayanan, S., J. Wang, and X. Lin, 2004, Phys. Rev. Lett. 93, 135503.

Orr, B. G., H. M. Jaeger, and A. M. Goldman, 1985, Phys. Rev. B 32, 7586 .

Orr, B. G., H. M. Jaeger, A. M. Goldman, and C. G. Kuper, 1986, Phys. Rev. Lett 56, 378.

Ovchinnikov, Y. N., 1973, Zh. Eksp. Teor. Fiz. 64, 719, [Sov. Phys. JETP, 37, 366 (1973)].

Ovchinnikov, Y. N., and V. Z. Kresin, 2005a, Eur. Phys. J B 45, 5 .

Ovchinnikov, Y. N., and V. Z. Kresin, 2005b, Eur. Phys. J B 47, 333.

Pankov, S., and V. Dobrosavlijevic, 2005, Phys. Rev. Lett. 94.

Parthasarathy, R., X.-M. Lin, K. Elteto, T. F. Rosenbaum, and H. M. Jaeger, 2004, Phys. Rev. Lett. 92, 076801.

Parthasarathy, R., X.-M. Lin, and H. Jaeger, 2001, Phys. Rev. Lett. 87, 186807.

Pollak, M., and C. J. Adkins, 1992, Phil. Mag. B 65, 855.

Punnoose, A., and A. M. Finkelstein, 2005, Science 310, 289.

Radhakrishnan, V., C. K. Subramaniam, S. Sankaranaranyanan, G. V. S. Rao, and R. Srinivasan, 1990, Physica
C 167, 53 .

Ralph, D., C. Black, and M. Tinkham, 1995, Phys. Rev. Lett. 74, 3241 .

Romero, H., and M. Drndic, 2005, Phys. Rev. Lett 95, 156801.

Rotkina, L., S. Oh, J. Eckstein, and S. Rotkin, 2005, Phys. Rev. B 72, 233407.

Sachdev, S., 2001, Quantum phase transitions (Cambridge university press).

Schmid, A., 1983, Phys. Rev. Lett. 51, 1506.

Schön, G., and A. Zaikin, 1990, Phys. Rep. 198, 237.

Shapira, Y., and G. Deutscher, 1983, Phys. Rev. B 27, 4463.

Shklovskii, B. I., 1973, Fiz. Tekh. Poluprovodn. (S.Petersburg) 6, 2335.

Shklovskii, B. I., and A. L. Efros, 1988, Electronic properties of Doped Semiconductors (Springer-Verlag, New York).

Simanek, E., 1979, Sol. State. Comm. 31, 419.

Simanek, E., 1994, Nonhomogeneous Superconductors (Oxford University Press, Oxford).

Simánek, E., and R. Brown, 1986, Phys. Rev. B 34, 3495.

Simon, R. W., B. J. Dalrymple, D. V. Vechten, W. W. Fuller, and S. A. Wolf, 1987, Phys. Rev. B 36, 1962.

Smith, R. A., and V. Ambegaokar, 1996, Phys. Rev. Lett. 77, 4962.

Sondhi, S., S. Girvin, J. Carini, and D. Shahar, 1997, Rev. Mod. Phys. 69, 315.

Thompson, R. S., 1970, Phys. Rev. B 1, 327.

Tinkham, M., 1996, Introduction to Superconductivity (McGraw-Hill, New York).

Tran, T., I. S. Beloborodov, X. M. Lin, V. M. Vinokur, and H. M. Jaeger, 2005, Phys. Rev. Lett. 95, 076806.

Usadel, K. D., 1970, Phys. Rev. Lett. 25, 507.

Wegner, F., 1979, Z. Phys. B 35, 207.

Wehrenberg, B. L., C. Wang, and P. J. Guyot-Sionnest, 2002, Phys. Chem. B 106, 10634.

Yakimov, A. I., A. V. Dvurechenskii, A. I. Nikiforov, and A. A. Bloshkin, 2003, 77, 445, [Sov. Phys. JETP Lett. 77, 376 (2003)].

Yu, D., C. Wang, B. L. Wehrenberg, and P. Guyot-Sionnest, 2004, Phys. Rev. Lett. 92, 216802.

Yu, X., P. M. Duxbury, G. Jeffers, and M. A. Dubson, 1991, Phys. Rev. B 44, 13163.

van der Zant, H. S. J., F. C. Fritschy, W. J. Elion, L. J. Geerlings, and J. E. Mooij, 1992, Phys. Rev. Lett 69, 2971.

Zhang, J., and B. I. Shklovskii, 2004, Phys. Rev. B 70, 115317.

Zhu, F., K. Zhang, E. Guenter, and C. Jin, 2000, Thin Solid Films 363, 314. 